\title{
Learning Force Fields from Stochastic Trajectories
}

\author{
Anna Frishman* \\ Department of Physics, Technion Israel Institute of Technology, 32000 Haifa, Israel \\ and Princeton Center for Theoretical Science, Princeton University, Princeton, New Jersey 08544, USA \\ Pierre Ronceray ${ }^{\dagger}$ \\ Center for the Physics of Biological Function, Princeton University, Princeton, New Jersey 08544, USA \\ and Princeton Center for Theoretical Science, Princeton University, Princeton, New Jersey 08544, USA
}

(Received 20 February 2019; revised manuscript received 26 September 2019; accepted 31 January 2020; published 13 April 2020)

\begin{abstract}
When monitoring the dynamics of stochastic systems, such as interacting particles agitated by thermal noise, disentangling deterministic forces from Brownian motion is challenging. Indeed, we show that there is an information-theoretic bound, the capacity of the system when viewed as a communication channel, that limits the rate at which information about the force field can be extracted from a Brownian trajectory. This capacity provides an upper bound to the system's entropy production rate and quantifies the rate at which the trajectory becomes distinguishable from pure Brownian motion. We propose a practical and principled method, stochastic force inference, that uses this information to approximate force fields and spatially variable diffusion coefficients. It is data efficient, including in high dimensions, robust to experimental noise, and provides a self-consistent estimate of the inference error. In addition to forces, this technique readily permits the evaluation of out-of-equilibrium currents and the corresponding entropy production with a limited amount of data.
\end{abstract}

DOI: $10.1103 /$ PhysRevX.10.021009

Subject Areas: Soft Matter, Statistical Physics

\section{INTRODUCTION}

From nanometer-scale proteins to micron-scale colloids, particles in biological and soft-matter systems undergo Brownian dynamics [1,2]: Their deterministic motion due to the forces competes with the random diffusion due to thermal noise from the solvent. At a larger scale, the overdamped Langevin equation describing Brownian dynamics is commonly used as an effective model for the stochastic evolution of complex systems such as motile cells [3], financial markets [4], or climate dynamics [5], where the noise corresponds to the random influence of fast, unresolved degrees of freedom, while force fields model persistent, deterministic trends. In the absence of forces, all trajectories would thus look alike [Fig. 1(a)]: The force field simultaneously shapes a system's trajectory [Figs. 1(b) and 1(c)] and encompasses most physical information about the system. The inference of such force fields from experimental data is therefore crucial to

\footnotetext{
*frishman@technion.ac.il

†onceray@princeton.edu
}

Published by the American Physical Society under the terms of the Creative Commons Attribution 4.0 International license. Further distribution of this work must maintain attribution to the author(s) and the published article's title, journal citation, and DOI.

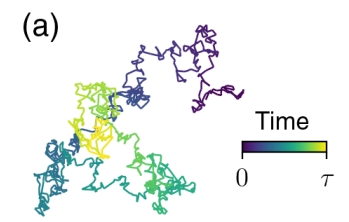

(b)

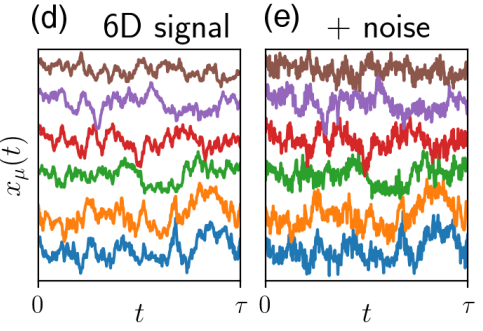

(c)
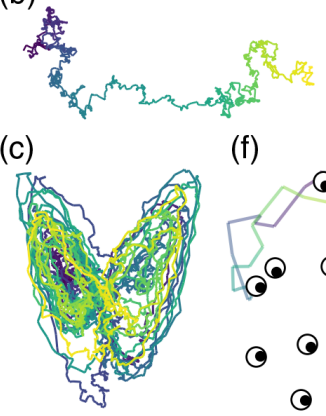

(f)

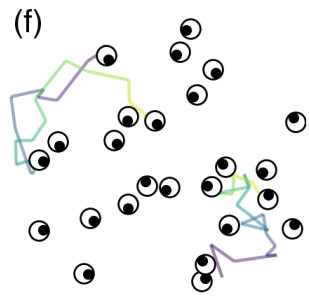

(g)

FIG. 1. Typical trajectories of example Brownian systems studied in this article. (a) Pure Brownian motion in 2D without forces. (b) A drifted Brownian motion trajectory. (c) The stochastic Lorenz process (see Fig. 5). (d) Time series of a 6D outof-equilibrium Ornstein-Uhlenbeck process (see Fig. 4). (e) The same trajectories as in (d), with additional time-uncorrelated measurement noise. (f) Self-propelled active Brownian particles with soft repulsion and harmonic confinement (see Fig. 7). (g) Simulated single-molecule trajectories in a complex environment with space-dependent diffusion (see Fig. 9). 
problems as varied as understanding the dynamics of single molecules in complex cellular environments [6,7], quantifying the interactions between colloidal particles [8], calibrating devices to optically trap particles [9], or identifying the laws governing the motion of cells [10]. This problem is particularly relevant in the context of living or driven out-of-equilibrium systems, where active forces induce dissipative currents at the mesoscale [11]. The knowledge of the force field in such cases would allow one to measure the mean entropy production rate and thus quantify the irreversibility of the dynamics, a question which gained attention recently [12-18]. Moreover, it would also enable one to measure the fluctuations of heat, work, and entropy production-the subject of stochastic thermodynamics [19] — which is so far only possible in highly controlled systems [20].

Numerous previous studies have proposed methods to reconstruct force fields motivated by applications in soft matter [21,22], cell biology [23-25], climate dynamics [26,27], finance [28-32], and other complex systems [33]. However, force inference in Brownian systems remains a hard problem, and a general method is still missing, in particular one addressing the many challenges associated with experimental data in soft-matter and biological systems. First, there needs to be enough information about the force available in the trajectory: Short trajectories are dominated by noise [Fig. 1(a)], and only after a long enough observation time does the effect of the force field become apparent [Fig. 1(b)]. Second, one needs a practical method to extract that information and reconstruct the force field, which is challenging for out-of-equilibrium systems with a complex spatial structure [Fig. 1(c)], in particular for high-dimensional processes [Figs. 1(f)] and in the presence of measurement error [Fig. 1(e)] and multiplicative noise [Fig. 1(g)].

Here we address these challenges for steady-state Brownian trajectories. We first use communication-theory tools to quantify the maximal rate at which information about a force field can be inferred from a trajectory (Sec. II). We relate this rate, that we term channel capacity of the system, to the entropy production rate, thus providing a novel link between stochastic thermodynamics and information theory. We then propose a practical procedure, stochastic force inference (SFI), to use the information in a trajectory and reconstruct the force field by projecting it onto a finite-dimensional functional space (Sec. III). By inferring the information contained in a trajectory, we propose a practical criterion to control overfitting, an aspect generally overlooked by previous approaches. We ensure that this method is robust to the presence of experimental noise. Finally, the diffusion coefficient can depend on the state of the system, which significantly complicates force inference: In such cases, we adapt our method to infer the space-dependent diffusion and force field (Sec. IV). Using simple model stochastic processes, we demonstrate that our method permits a quantitative evaluation of phase-space forces, currents, and diffusion coefficients, and estimate the entropy production with a minimal amount of data.

We focus in this article on stochastic systems governed by the overdamped Langevin equation, where friction dominates over inertia, as is typically the case in subcellular biological systems, for instance. We thus consider a system where the phase-space coordinates $x_{\mu}$ obey Brownian dynamics,

$$
\dot{x}_{\mu}=F_{\mu}(\mathbf{x})+\sqrt{2 D}_{\mu \nu} \xi_{\nu},
$$

where $F_{\mu}(\mathbf{x})$ is the force field (we absorb the mobility matrix in its definition), $D_{\mu \nu}$ is the diffusion tensor, and $\xi_{\mu}$ is a Gaussian white noise, $\left\langle\xi_{\mu}(t) \xi_{\nu}\left(t^{\prime}\right)\right\rangle=\delta\left(t-t^{\prime}\right)$, and we use the Einstein convention of summation over repeated indices throughout. In Secs. II and III of this article, we assume that $D_{\mu \nu}$ is space independent and known; in Sec. IV, we address the case of inhomogeneous diffusion, which modifies Eq. (1).

\section{THE INFORMATION CONTENT OF BROWNIAN TRAJECTORIES}

We propose to interpret Brownian dynamics [Eq. (1)] as a noisy transmission channel, where the force is the encoded signal, and $\sqrt{2 \mathbf{D}} \xi$ is the noise (Fig. 2). Information can be read out from such a channel at a maximal rate $C$ called the channel capacity, which relates to the signal-to-noise ratio of the input [34]. This finite capacity fundamentally limits the ability to infer forces by monitoring the dynamics. To build up intuition, consider the simplest case of a spatially constant force with isotropic diffusion, corresponding to drifted Brownian motion [Fig. 1(b)]. The capacity is then given by $C=F^{2} / 4 D$ (expressed in natural information units, or nats, per time unit -1 nat $=1 / \log 2$ bits). The force to infer here is equal to the persistent velocity, which can be estimated as $\hat{F}_{\mu}=\Delta x_{\mu} / \tau$, where $\Delta \mathbf{x}$ is the end-to-end vector along the trajectory of duration $\tau$. The relative error on this estimator due to random diffusion is $\left\langle\|\hat{\mathbf{F}}-\mathbf{F}\|^{2} / F^{2}\right\rangle=$ $2 d D / \tau F^{2}=d / 2 I$, where $d$ is the space dimension. We identify here $I=C \tau$, defining it as the information in the trajectory. Persistent motion thus starts to emerge from the noise if the trajectory duration $\tau$ is longer than $d / C$, corresponding to the diffusive-to-persistent transition for the mean-squared displacement. Equivalently, the force starts to be resolved if $I>d$, i.e., if more than one bit of information is available for each degree of freedom $\hat{F}_{\mu}$ to infer.

We now give a precise meaning to the notion of capacity for general Brownian systems, where interparticle interactions and external fields lead to a force that depends on the state $\mathbf{x}$ of the system in phase space. We recognize that within communication theory, the dynamics of a Brownian system [Eq. (1)] corresponds to an infinitebandwidth Gaussian channel [34]. The signal transmitted 


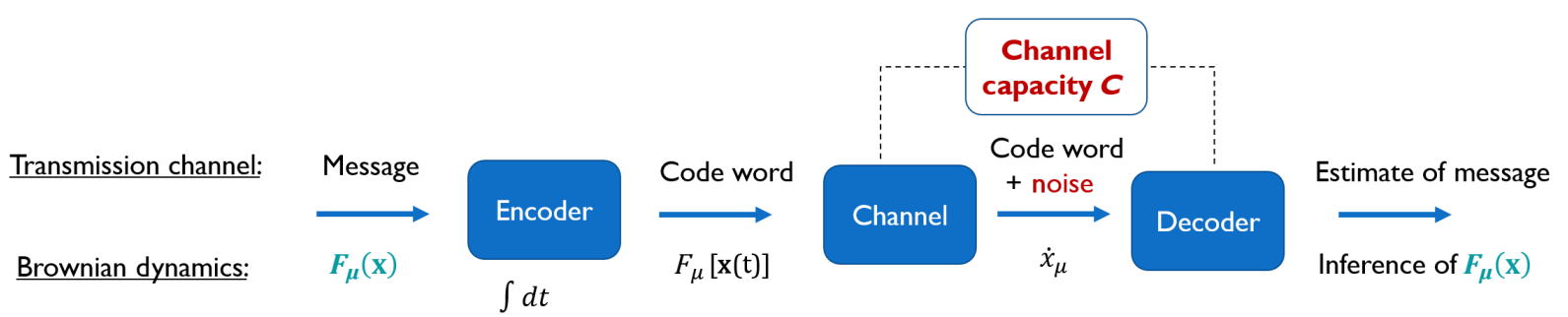

FIG. 2. The dynamics of an overdamped system can be seen as a noisy data-transmission channel encoding information about the force field, with a rate bounded by the channel capacity $C$ as defined in Eq. (2). Note that this definition does not include the information loss stemming from the measurement device. This analogy is further discussed in the Appendix A.

is the force, with signal power equal to its time-averaged square. The corresponding channel's capacity, which we refer to as the system's capacity, is thus (see Appendix A)

$$
C=\frac{1}{4} \int F_{\mu}(\mathbf{x}) D_{\mu \nu}^{-1} F_{\nu}(\mathbf{x}) P(\mathbf{x}) d \mathbf{x}
$$

where $P(\mathbf{x})$ is the steady-state probability distribution function of the process. This quantity was previously considered as a penalty term to regularize force inference [35].

The steady-state Fokker-Planck equation allows us to decompose the force into a sum of two terms,

$$
F_{\mu}=v_{\mu}+D_{\mu \nu} \partial_{\nu} \log P,
$$

where $v_{\mu}$ is the average phase-space velocity quantifying the presence of irreversible currents, and $D_{\mu \nu} \partial_{\nu} \log P$ quantifies reversible, diffusive currents. Interestingly, this decomposition implies that the capacity defined in Eq. (2) splits into two non-negative parts, one related to dissipation and the other to spatial structure, as

$$
4 C=\dot{S}+G
$$

Here, $\dot{S}$ is the steady-state entropy production of the process [19], $\dot{S}=\int v_{\mu} D_{\mu \nu}^{-1} v_{\nu} P(\mathbf{x}) d \mathbf{x}$ (we set the Boltzmann constant $k_{B}=1$ throughout). In the case of thermal systems satisfying the Einstein relation, $\dot{S}$ corresponds to the rate at which the system dissipates heat into the bath, divided by the temperature; in other cases, $\dot{S}$ quantifies the irreversibility of the dynamics. The second term named inflow rate $G=\int g_{\mu} D_{\mu \nu} g_{\nu} P(\mathbf{x}) d \mathbf{x}$ with $g_{\mu}=\partial_{\mu} \log P$ was previously introduced and studied in Ref. [36]. It reflects the amount of information that the force field injects into the system in order to maintain probability gradients against diffusion and is positive even at equilibrium. Indeed, in a thought experiment where the force field would be suddenly switched off, $G$ would correspond to the instantaneous entropy production rate due to the relaxation of probability gradients (see Appendix B 2). The inflow rate quantifies the fact that in steady state, the system dwells in convergent regions of the force field: An equivalent expression for it is indeed [36] $G=-\int \partial_{\mu} F_{\mu}(\mathbf{x}) P(\mathbf{x}) d \mathbf{x}$. In a deterministic system, it would thus correspond to the average phasespace contraction rate. The connection between the inflow rate and the previously introduced notions of traffic and frenesy [37,38] is explored in Appendix B 3. As $G \geq 0$, Eq. (4) provides a generic upper bound to the entropy production in Brownian systems, $\dot{S} \leq 4 C$.

The decomposition of the information into dissipative and structural contributions introduced in Eq. (4) can be expressed at the level of individual trajectories in phase space. Indeed, the entropy production rate corresponds to the rate at which trajectories $\mathcal{C}=\{\mathbf{x}(t)\}_{t=0 \ldots \ldots \tau}$ become distinguishable from their time-reversed version $-\mathcal{C}=\{\mathbf{x}(\tau-t)\}_{t=0, \ldots, \tau}$, as quantified by the Kullback-Leibler divergence rate [19]: $\dot{S}=\lim _{\tau \rightarrow \infty}(1 / \tau)\langle\log \mathcal{P}(\mathcal{C} \mid F) / \mathcal{P}(-\mathcal{C} \mid F)\rangle_{F}$. Here, $\mathcal{P}(\mathcal{C} \mid F)$ is the probability that the system follows a trajectory $\mathcal{C}$ under Brownian dynamics [Eq. (1)] in the force field $F$, and $\langle\cdot\rangle_{F}$ corresponds to averaging over all possible trajectories $\mathcal{C}$ with weight $\mathcal{P}(\mathcal{C} \mid F)$. Time reversal $(\mathcal{C}, F) \mapsto(-\mathcal{C}, F)$ changes the sign of the heat produced along the trajectory, and thus connects dissipation and irreversibility of the dynamics. Interestingly, a similar expression can be derived for the inflow rate [36]: $G=$ $\lim _{\tau \rightarrow \infty}(1 / \tau)\langle\log \mathcal{P}(\mathcal{C} \mid F) / \mathcal{P}(-\mathcal{C} \mid-F)\rangle_{F}$, where $-F$ corresponds to the reversed force field. Indeed, the operation $(\mathcal{C}, F) \mapsto(-\mathcal{C},-F)$ now leaves the heat unchanged but reverses the sign of the divergence of the force. At equilibrium, this operation corresponds to inverting the energy landscape: For a typical trajectory that dwells in potential wells, the reverse trajectory is atypical in the force field $-F$, as it spends time around unstable maxima of energy. Finally, the capacity can be expressed as $4 C=$ $\lim _{\tau \rightarrow \infty}(1 / \tau)\langle\log \mathcal{P}(\mathcal{C} \mid F) / \mathcal{P}(\mathcal{C} \mid-F)\rangle_{F}$ : This operation reverses both heat and force divergence. Intuitively, there is information about the force in a trajectory if it allows one to distinguish the force field from its reverse. More naturally, the capacity quantifies the rate at which a trajectory becomes distinguishable from force-free Brownian motion: Indeed, it can be written as $C=\lim _{\tau \rightarrow \infty}(1 / \tau)\langle I(\mathcal{C})\rangle_{F}$, where we define 


$$
I(\mathcal{C})=\log \frac{\mathcal{P}(\mathcal{C} \mid F)}{\mathcal{P}(\mathcal{C} \mid 0)}
$$

as the trajectory-wise information gain about the force field.

\section{STOCHASTIC FORCE INFERENCE}

A trajectory of finite duration contains finite information quantified by Eq. (5). We now introduce a practical method, stochastic force inference (SFI), that uses this information to reconstruct the force field. In contrast with the drifted Brownian motion, a spatially variable force field is, in principle, characterized by an infinite number of degrees of freedom: the force value at each point in space. With a finite trajectory, only a finite number of combinations of degrees of freedom can be estimated. It is therefore natural to approximate the force field as a linear combination of a finite basis of $n_{b}$ known functions $b=\left\{b_{\alpha}(\mathbf{x})\right\}_{\alpha=1, \ldots, n_{b}}$. The force can, in principle, be approximated arbitrarily well by using a large enough set of functions from a complete basis, such as polynomials, wavelets or Fourier modes. Alternatively, a limited number of functions might suffice if an educated guess of the functional form of the force field can be made. We propose to perform this approximation by projecting the force field onto the space spanned by $b_{\alpha}(\mathbf{x})$ using the steady-state probability distribution function $P$ as a measure. This approximation corresponds to a least-squares fit of the force field by linear combinations of the $b_{\alpha}$ 's. To this aim, we define the projector $c_{\alpha}(\mathbf{x})=B_{\alpha \beta}^{-1 / 2} b_{\beta}(\mathbf{x})$, where $B_{\alpha \beta}$ is an orthonormalization matrix such that $\int c_{\alpha} c_{\beta} P(\mathbf{x}) d \mathbf{x}=\delta_{\alpha \beta}$. Our approximation of the force field is then $F_{\mu}(\mathbf{x}) \approx F_{\mu \alpha} c_{\alpha}(\mathbf{x})$ with the projection coefficient

$$
F_{\mu \alpha}=\int F_{\mu}(\mathbf{x}) c_{\alpha}(\mathbf{x}) P(\mathbf{x}) d \mathbf{x} .
$$

This approach is akin to projecting the dynamics onto a finite-dimensional subchannel of capacity $C_{b}=$ $\frac{1}{4} D_{\mu \nu}^{-1} F_{\mu \alpha} F_{\nu \alpha}<C$. Similarly, we can define the projection $v_{\mu \alpha}$ of the phase-space velocity. The corresponding entropy production $\dot{S}_{b}=D_{\mu \nu}^{-1} v_{\mu \alpha} v_{\nu \alpha}$ is then a lower bound to the total entropy production. Interestingly, for a system obeying Brownian dynamics [Eq. (1)] but where only a subset of degrees of freedom can be observed, our framework gives the force averaged over hidden variables and provides a lower bound on the entropy production limited to the observable currents (see Appendix E).

The projected force field has a finite number of degrees of freedom $N_{b}=d n_{b}$, one per element of the $d \times n_{b}$ tensor $F_{\mu \alpha}$, and corresponds to a finite capacity $C_{b}$. Inferring the approximate force with a finite trajectory thus becomes, in principle, possible when the information $I_{b}=\tau C_{b}>$ $N_{b}$. However, the force coefficients introduced in Eq. (6) are not directly accessible from experimental data. Indeed, neither the force nor the probability distribution function $P$ are known, the latter being also required in the definition of the orthonormal projectors $c_{\alpha}$. Instead, the available data are typically a discrete time series $\mathbf{x}\left(t_{i}\right)$ of phase-space positions at sampling times $t_{i}=i \Delta t$. We thus propose to estimate phase-space averages by discrete time integrals along the trajectory. The empirical projectors are defined as $\hat{c}_{\alpha}=\hat{B}_{\alpha \beta}^{-1 / 2} b_{\beta}$, with $\hat{B}_{\alpha \beta}=\sum_{i} b_{\alpha}\left(\mathbf{x}\left(t_{i}\right)\right) b_{\beta}\left(\mathbf{x}\left(t_{i}\right)\right)(\Delta t / \tau)$. Furthermore, the force can be expressed in terms of a local Itô average of $\dot{\mathbf{x}}$ [39]: A local estimator for the force at $\mathbf{x}\left(t_{i}\right)$ is thus $\Delta \mathbf{x}\left(t_{i}\right) / \Delta t$, with $\Delta \mathbf{x}\left(t_{i}\right)=\mathbf{x}\left(t_{i+1}\right)-\mathbf{x}\left(t_{i}\right)$. Combining these two insights yields an operational definition for the estimator of Eq. (6) in terms of a discrete Itô integral (see Appendix C),

$$
\hat{F}_{\mu \alpha}=\frac{1}{\tau} \sum_{i} \Delta x_{\mu}\left(t_{i}\right) \hat{c}_{\alpha}\left(\mathbf{x}\left(t_{i}\right)\right),
$$

which is the discretized version of the Itô integral $(1 / \tau) \int_{0}^{\tau} \hat{c}_{\alpha}(\mathbf{x}(t)) d x_{\mu}(t)$. Indeed, discretizing Eq. (1) yields $\Delta \mathbf{x}\left(t_{i}\right)=\mathbf{F}\left(\mathbf{x}\left(t_{i}\right)\right) \Delta t+\sqrt{2 \mathbf{D}} \Delta \xi_{i}$, where $\Delta \xi_{i}$ is independent of $\mathbf{x}\left(t_{i}\right)$ : In the long-trajectory limit, the main contribution comes from the force, while the noise averages to zero. Equation (7) corresponds to a linear regression of the local force estimator, previously suggested for one-dimensional systems [29], and coincides with the maximumlikelihood estimator of the force projection coefficients. The typical squared relative error on the inferred coefficients due to the diffusive noise can be estimated in practice as $\delta \hat{F}^{2} / \hat{F}^{2} \sim N_{b} / 2 \hat{I}_{b}$ (see Appendix C), where $\hat{I}_{b}=(\tau / 4) D_{\mu \nu}^{-1} \hat{F}_{\mu \alpha} \hat{F}_{\nu \alpha}$ is the empirical estimate of information contained in the trajectory. This formula indicates that again, in order to resolve the force coefficients, the information in the trajectory should exceed the number of inferred parameters. Another source of error stems from the fact that the force varies over a finite time step $\Delta t$; we provide an estimator for the magnitude of the resulting bias in Appendix F.

We now demonstrate the utility of our method using simulated data of simple models. The simplest spatially varying force field is a harmonic trap, i.e., an OrnsteinUhlenbeck process (Fig. 3). We benchmark our method by using a first-order polynomial basis $b=\left\{1, x_{\mu}\right\}$, which can capture the exact force field. The 2D trajectory displayed in Fig. 3(a) has an information content of $I=27.6$ bits, while this linear channel has $N_{b}=6$ degrees of freedom, allowing precise inference of the projected force field [Fig. 3(a)]. Indeed, the squared relative error on the force coefficients is 0.15 ; this is consistent with the operational estimate of this error, $N_{b} / 2 \hat{I}_{b}=0.16$. The force along the trajectory is thus inferred to a good approximation [Fig. 3(a) inset]. Furthermore, the projected force field $\hat{F}_{\mu \alpha} \hat{c}_{\alpha}(\mathbf{x})$ provides an ansatz that can be extrapolated beyond the trajectory [Fig. 3(a)], which works equally well here as the functional form of the force field is fully 

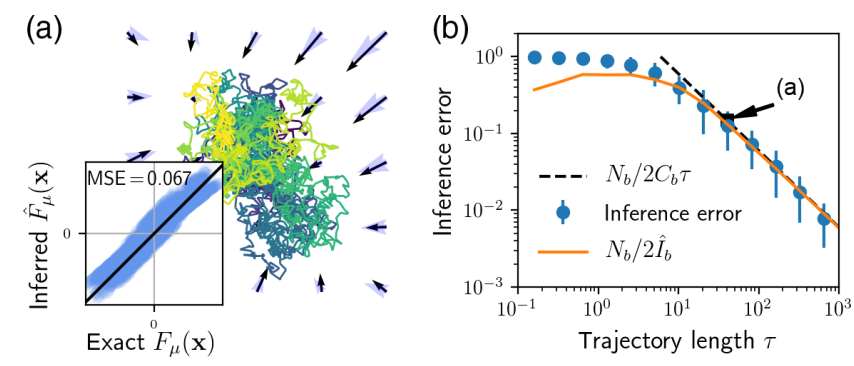

FIG. 3. Stochastic force inference for a 2D Ornstein-Uhlenbeck process with force field $F_{\mu}(\mathbf{x})=-\Omega_{\mu \nu} x_{\nu}$ and isotropic diffusion. (a) An example trajectory. The inferred force field for this trajectory using SFI with functions $b=\left\{1, x_{\mu}\right\}$ (blue arrows) is compared to the exact force field (black arrows). Inset: The inferred force components along the trajectory versus the exact force components with normalized mean-squared error (MSE). (b) The average of the relative error $\left[\left(\hat{F}_{\mu \alpha}-F_{\mu \alpha}^{\tau}\right) D_{\mu \nu}^{-1}\left(\hat{F}_{\nu \alpha}-F_{\nu \alpha}^{\tau}\right)\right] /\left[\hat{F}_{\mu \alpha} D_{\mu \nu}^{-1} \hat{F}_{\nu \alpha}\right]$ on the inferred projection coefficients $\hat{F}_{\mu \alpha}$ and its self-consistent estimate $N_{b} / 2 \hat{I}_{b}$ both converge to $N_{b} / 2 I_{b}$, as expected from theory (see Appendix C). Here, $F_{\mu \alpha}^{\tau}=\int F_{\mu}(\mathbf{x}(t)) \hat{c}_{\alpha}(\mathbf{x}(t))(d t / \tau)$ is the projection of the exact force on the empirical projectors.

captured by our choice of basis. More quantitatively, we confirm the predicted behavior for the squared relative error by studying an ensemble of trajectories [Fig. 3(b)].

In the case of out-of-equilibrium Brownian systems, our method also permits the approximation of phase-space currents and entropy production. Indeed, the phase-space velocity $\mathbf{v}$ can be expressed in terms of a local Stratonovich average of $\dot{\mathbf{x}}$, reflecting the fact that it is odd under time reversal [40]. Our estimator for the projection coefficients of the phase-space velocity is thus (see Appendix D)

$$
\hat{v}_{\mu \alpha}=\frac{1}{\tau} \sum_{i} \Delta x_{\mu}\left(t_{i}\right) \hat{c}_{\alpha}\left(\frac{\mathbf{x}\left(t_{i+1}\right)+\mathbf{x}\left(t_{i}\right)}{2}\right)
$$

which is the discretized version of the Stratonovich integral $(1 / \tau) \int_{0}^{\tau} \hat{c}_{\alpha}(\mathbf{x}(t)) \circ d x_{\mu}(t)$. This allows the inference of the entropy production rate $\hat{\dot{S}}_{b}=D_{\mu \nu}^{-1} \hat{v}_{\mu \alpha} \hat{v}_{\nu \alpha}$ associated with the observed currents. This estimator for the entropy production rate is biased, with an error that can be self-consistently controlled as $\hat{\dot{S}}_{b}=\dot{S}_{b}+2 N_{b} / \tau+O\left\{\left(2 \hat{\dot{S}}_{b} / \tau+\left(2 N_{b} / \tau\right)^{2}\right)^{1 / 2}\right\}$ : The entropy production rate in the channel can thus be inferred using a single trajectory provided that several $k_{B}$ 's per degree of freedom are dissipated.

The simplest structure for phase-space currents corresponds to cyclic circulation around a point. The detection of such features in active biological systems has been the focus of a number of recent studies which employ phasespace coarse graining $[11,14,17]$. This method is, however, limited to low-dimensional systems, and even then requires large amounts of data: Indeed, the capacity per degree of freedom is low, as each grid cell is visited infrequently. In contrast, our method provides a way to detect circulation in any dimension with minimal data. Using the centered linear basis $b_{\alpha}(\mathbf{x})=\bar{x}_{\alpha}=x_{\alpha}-\int x_{\alpha}(d t / \tau)$, we can infer the velocity coefficients $\hat{v}_{\mu \alpha}$, which have a matrix structure. This matrix reads $\hat{v}_{\mu \alpha}=C_{\alpha \beta}^{-1 / 2} A_{\beta \mu}$, where $C_{\mu \nu}=\int \bar{x}_{\mu} \bar{x}_{\nu}(d t / \tau)$ is the covariance matrix, and the antisymmetric part of $A_{\mu \nu}$ is $A_{\{\mu \nu\}}=(1 / 2 \tau) \int \bar{x}_{\mu} d x_{\nu}-\bar{x}_{\nu} d x_{\mu}$, which is the rate at which the process encircles area in the $(\mu, \nu)$ plane $[18,41]$. This rate, sometimes called probability angular momentum $[42,43]$, intuitively quantifies circulation and closely connects to cycling frequencies $[15,44]$. Indeed, the eigenvectors of $A_{\{\mu \nu\}}$ can be used to define cycling planes (see Appendix $\mathrm{H}$ ). The entropy production rate due to cycling reads $\hat{\dot{S}}_{b}=D_{\mu \nu}^{-1} A_{\nu \rho} C_{\rho \sigma}^{-1} A_{\sigma \mu}$.

We demonstrate the potency of our cycle-detection method on a challenging dataset: a short trajectory of an out-of-equilibrium Ornstein-Uhlenbeck process in dimension $d=6$ [Fig. 4(a)], which is equivalent to popularly used bead-spring models $[14,16,44]$. Our method identifies the principal circulation plane accurately, together with the force field [Fig. 4(c)]. Quantitatively, we demonstrate that the angular error in the identification of this plane vanishes with increasing trajectory length [Fig. 4(e)], concomitant with the convergence of $\hat{\dot{S}}_{b}$ to the exact value [Fig. 4(f)]. The entropy production inferred is associated with the observable currents: If only a fraction of the degrees of freedom can be observed, $\hat{\dot{S}}_{b}$ is a lower bound to the total entropy production of the system [Fig. 4(g)], as some currents are not observable. In particular, if only 1 degree of freedom can be measured, this technique will yield $\hat{\hat{S}}_{b}=0$; alternative techniques based on the non-Markovianity of the dynamics are better suited to inferring entropy production in this case [45].

A major challenge in the inference of dynamical properties of stochastic systems from real data is timeuncorrelated measurement noise, which often dominates time derivatives of the signal. Indeed, in our inference scheme, Eq. (7) is highly sensitive to such a noise. In contrast, the time-reversal antisymmetry of the velocity coefficients $\hat{v}_{\mu \alpha}$ makes them robust against measurement noise (see Appendix F). Exploiting this symmetry, we obtain an unbiased estimator for the force by using the relation between Itô and Stratonovich integration,

$$
\hat{F}_{\mu \alpha}=\hat{v}_{\mu \alpha}+D_{\mu \nu} \hat{g}_{\nu \alpha},
$$

where $\hat{g}_{\mu \alpha}=-\sum_{i}(\Delta t / \tau) \partial_{\mu} \hat{c}_{\alpha}\left(\mathbf{x}\left(t_{i}\right)\right)$ is an estimator for the projection of $g_{\mu}=\partial_{\mu} \log P$ onto the basis [note that while $\hat{g}_{\mu}(\mathbf{x}) \equiv \hat{g}_{\mu \alpha} \hat{c}_{\alpha}(\mathbf{x})$ is an estimate of $\partial_{\mu} \log P(\mathbf{x})$, it is not a gradient and thus cannot be integrated to estimate $P(\mathbf{x})$ ]. The modified estimator proposed in Eq. (9) can be computed only if the projection basis is smooth, and would not apply to grid coarse graining, for instance. It requires 


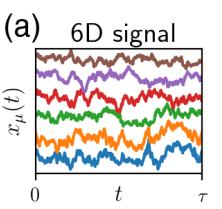

(b) + noise
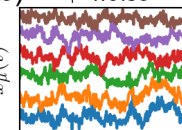

$0+t$

(c)
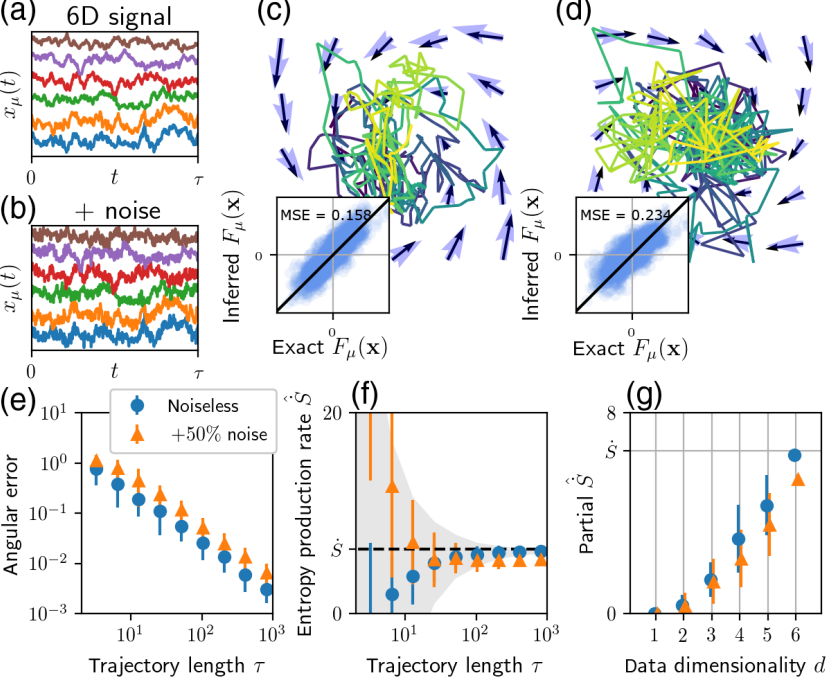

FIG. 4. (a) Time series of a 6D out-of-equilibrium OrnsteinUhlenbeck process, with anisotropic harmonic confinement and diffusion tensor, and circulation. The force field is $F_{\mu}(\mathbf{x})=$ $-\Omega_{\mu \nu} x_{\nu}$. The matrix $\Omega$ and the diffusion matrix are chosen from a random ensemble. The antisymmetric part of $D^{-1} \Omega$ has rank two, thus inducing circulation in a randomly chosen plane. (b) The same trajectories as in (d) with additional time-uncorrelated measurement noise. (c) SFI for the trajectory in (a) allows precise identification of the plane of circulation and reconstruction of the force along the trajectory. (d) SFI applied to the trajectory in (b) with measurement noise. It can still detect forces accurately. (e) Convergence of the angular error for cycle detection with increasing trajectory length for the process shown in (d) and (e). (f) Inferred entropy production rate for this process with and without measurement noise (we subtract here the systematic bias $\left.2 N_{b} / \tau\right)$. The shadowed area indicates the self-consistent confidence interval for the inferred entropy production. The dotted line shows the exact value of the entropy produced; for the noisy process, SFI underestimates this value due to blurring of the currents. (g) Entropy production captured when observing a $d$-dimensional projection of the trajectory averaged over direction of observation for long trajectories. In (e)-(g), the error bars indicate the standard deviation over an ensemble of 32 trajectories. Parameters of the simulations are presented in Appendix $\mathrm{H}$.

knowledge of the diffusion tensor $D_{\mu \nu}$, as we discuss in Sec. IV. Using this modified force estimator allows precise reconstruction of the force field, circulation, and entropy production even in the presence of large measurement noise [Figs. 4(b) and 4(g)]. The limiting factor on force inference due to measurement noise then becomes the blurring of the spatial structure of the process. For observations with a finite time step $\Delta t$, the currents are also blurred by time discretization, introducing an additional bias in the force estimator (see Appendix F) and resulting in an underestimate of the entropy production. Note, however, that this finite $\Delta t$ effect induces a bias on $\hat{v}_{\mu \alpha}$ only: For an equilibrium, time-reversible process, $\hat{v}_{\mu \alpha} \rightarrow 0$ and the force estimator reduces to $D_{\mu \nu} \hat{g}_{\nu \alpha}$, which is independent of the time ordering of the data.

We have so far considered only the case of linear systems projected onto linear functions. In general, force fields are
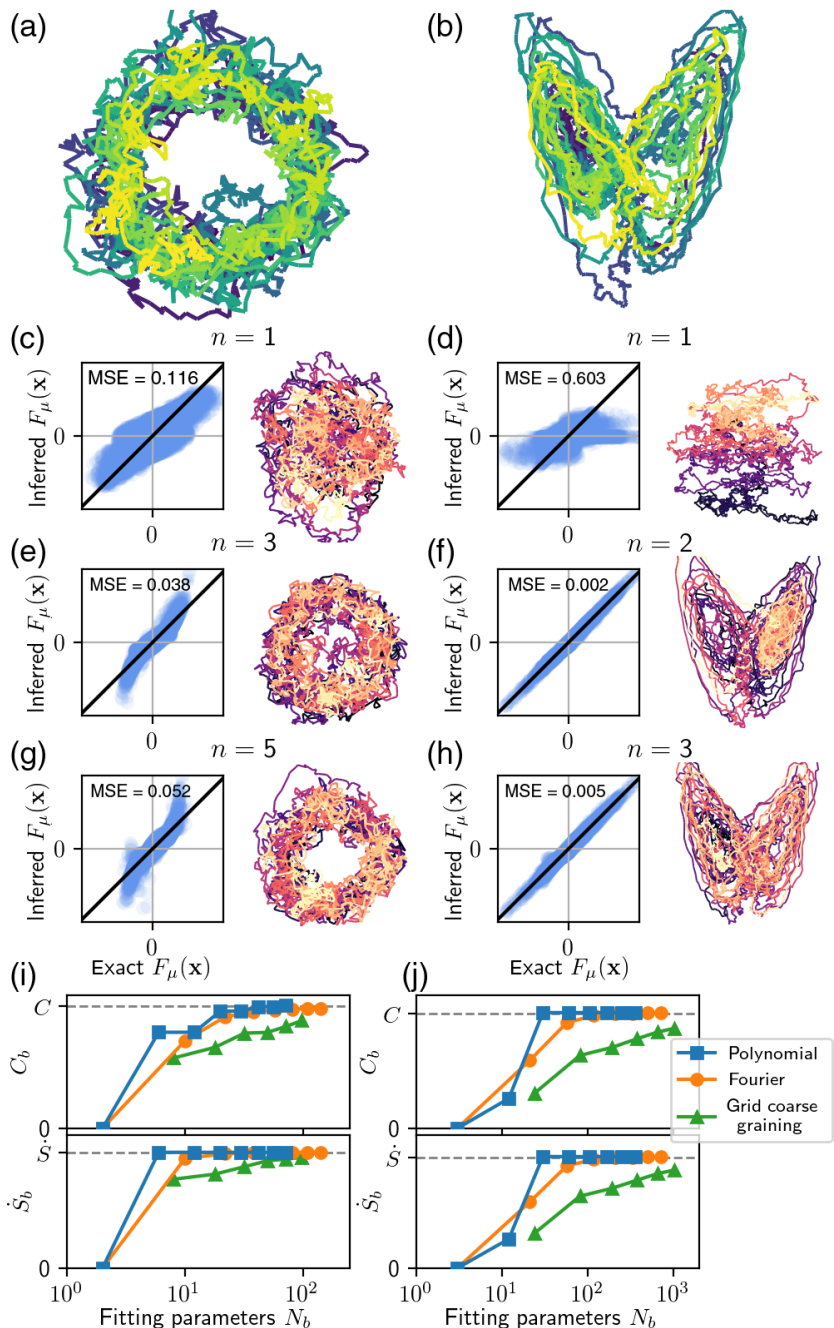

FIG. 5. Stochastic force inference with nonlinear force fields. (a) Trajectory of an out-of-equilibrium process with harmonic trapping and circulation, and a Gaussian repulsive obstacle in the center. The force field is given by $F_{\mu}(\mathbf{x})=-\Omega_{\mu \nu} x_{\nu}+\alpha e^{-x^{2} / 2 \sigma^{2}} x_{\mu}$ where $\Omega$ has both a symmetric and antisymmetric part. (b) Trajectory of the stochastic Lorenz process, a 3D process with a chaotic attractor. The force field is $F_{x}=s(y-x), F_{y}=$ $r x-y-z x, F_{z}=x y-b z$, where we choose $r=10, s=3$, and $b=1$. (c)-(h) SFI for these two trajectories, respectively, with polynomials of order $n=1,3,5$ and $n=1,2$, 3: inferred force versus exact force (left) and bootstrapped trajectory using the inferred force field (right). (i),(j) Capacity (top) and entropy production (bottom) of each process projected on different bases for an asymptotically long trajectory as a function of the number of degrees of freedom $N_{b}$ in the basis. These bases are polynomial and Fourier functions with order $n=0, \ldots, 7$, and a coarse-grained approximation with a variable number of grid cells $n=2, \ldots, 7$ in each dimension. Parameters and details of the simulations are presented in Appendix $\mathrm{H}$. 
nonlinear, which can result in a complex spatial structure. We illustrate this in Figs. 5(a) and 5(b) for processes with, respectively, nonpolynomial forces and a complex attractor [46]. For such processes, SFI with a linear basis captures the covariance of the data and the circulation of their current. However, it fails to reproduce finer features, as evident by inspecting bootstrapped trajectories generated using the inferred force field [Figs. 5(c) and 5(d)]. A better approximation of the force can be obtained by expanding the projection basis, for instance by including higher-order polynomials $\left\{x_{\mu} x_{\nu}\right\},\left\{x_{\mu} x_{\nu} x_{\rho}\right\} \ldots$ [Figs. 5(h)] or Fourier modes. The captured fraction of the capacity and entropy production increases monotonically when expanding the basis [Figs. 5(i) and 5(j)], corresponding to finer geometrical details: The force field is well resolved if the measured capacity does not increase upon further expansion of the basis. However, expanding the basis also results in an increase in the number of parameters to infer, which eventually leads to overfitting.

For a finite trajectory, there is therefore a trade-off between the precision of the inferred force and the completeness of the force-field representation. This is demonstrated in Figs. 6(a) and 6(b) by plotting the force inference error along the trajectory as a function of the
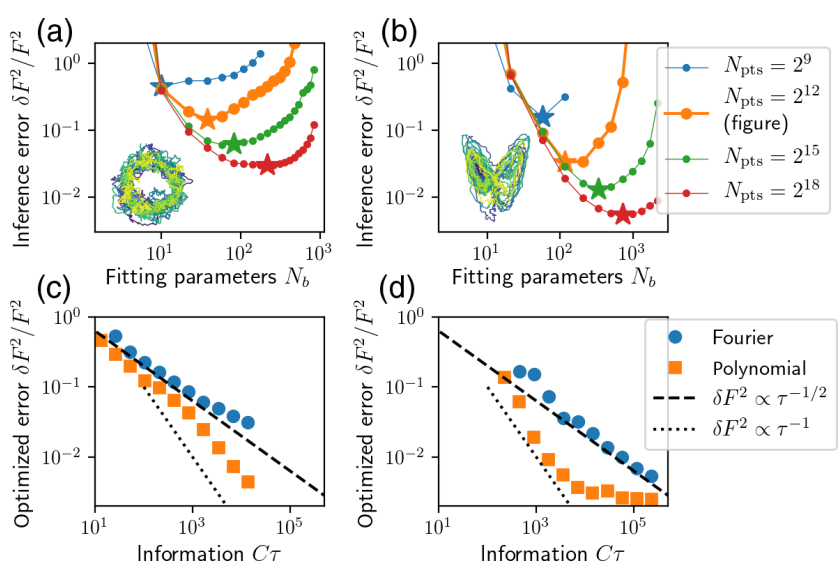

FIG. 6. Influence of the size of the basis on the precision of SFI. (a),(b) SFI error as a function of the number of fit parameters, respectively, for the models presented in Figs. 5(a) and 5(b) with a Fourier basis, and for different numbers of time steps in the trajectory. Specifically, the $y$ axis is the meansquared relative error on the inferred force along the trajectory, $\left\langle\left(\hat{F}_{\mu}-F_{\mu}\right) D_{\mu \nu}^{-1}\left(\hat{F}_{\nu}-F_{\nu}\right)\right\rangle /\left\langle\hat{F}_{\mu} D_{\mu \nu}^{-1} \hat{F}_{\nu}\right\rangle$. The crossover from under- to overfitting is apparent and takes place at larger $N_{b}$ and lower error with longer trajectories. The star symbols indicate the optimal basis size predicted by our self-consistent criterion of maximizing $\hat{I}_{b}-\delta \hat{I}_{b}$. (c),(d) The squared error as a function of the amount of information $C \tau$ in a trajectory of duration $\tau$ for the optimal basis, averaged over $n=3$ trajectories. For the Lorenz process with a polynomial basis [(d) orange squares], the convergence is fast as the basis is adapted to the exact force field, and the saturation of the error to a lower plateau is due to the finite time step (see Appendix F). number $N_{b}$ of degrees of freedom in the basis. At small $N_{b}$, this error decreases, as it mostly originates from underfitting. At large $N_{b}$, the error increases, as all statistically significant information is already captured, and adding new functions primarily fits the noise. This is reflected in the inferred information $\hat{I}_{b}$, which steadily increases with the number of fitting parameters $N_{b}$ : The increase is initially mainly due to the increase in the captured information $I_{b}$, but as $N_{b}$ grows, so does the typical error on $\hat{I}_{b}, \delta \hat{I}_{b} \approx \sqrt{2 \hat{I}_{b}+N_{b}^{2} / 4}$ (see Appendix C 4), and this error eventually overwhelms the gain in $I_{b}$. As a practical criterion to optimize between under- and overfitting and best estimate the force along the trajectory, we thus propose to use the basis $b$ which maximizes the information $I_{b}$ that can be statistically resolved. In practice, we find that choosing the basis size that maximizes $\hat{I}_{b}-\delta \hat{I}_{b}$ (i.e., the inferred information minus one standard deviation) robustly selects the optimal basis size for a given trajectory [star symbols in Figs. 6(a) and 6(b)]. An alternative optimization procedure based on a similar balance was suggested in Ref. [29] for one-dimensional processes. We empirically observe that when using this criterion to adapt the basis to the trajectory, the typical squared error on force inference scales as $\tau^{-1 / 2}$ with the trajectory duration $\tau$ [Figs. 6(c) and 6(d)]. There is an exception to this scaling: When the force field can be exactly represented by a finite number of functions of the basis, such as the Lorenz process with order 2 polynomials, this same criterion selects the smallest adapted basis: Further adding functions does not resolve more information. This results in a faster convergence of the force field as $\tau^{-1}$ [Fig. 6(d)], which is the rate of convergence of the force projections for a given basis size.

Systems with many degrees of freedom, such as active interacting particles [Fig. 7(a)], are challenging to treat. Indeed, with limited data, the criterion $\hat{I}_{b} \gg N_{b}$ precludes even the inference of gross features of the force field. In such cases, however, the use of symmetries can make the problem tractable. For instance, treating particles as identical implies that forces are invariant under particle exchange, which greatly reduces the number of parameters to infer. Forces can then be expanded as one-particle terms, pair interactions, and higher orders by choosing an appropriate basis (see Appendix H 6). With this scheme, a large number of particles actually results in enhanced statistics, allowing accurate inference of the force components [Figs. 7(a) and 7(b)] and reconstruction of the pair interactions [Fig. 7(c)] with a limited amount of data. This method could be straightforwardly extended to include, e.g., alignment interactions between particles. In contrast to standard methods to infer pair interaction potentials, we do not rely here on an equilibrium assumption.

\section{INHOMOGENEOUS DIFFUSION}

We have so far assumed that the diffusion tensor does not depend on the state of the system. While this is a natural 
(a)

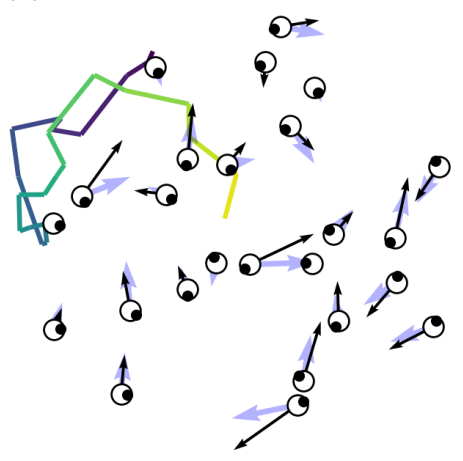

(b)

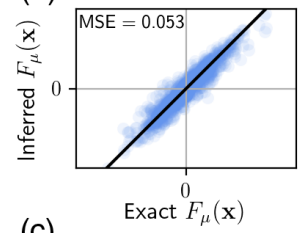

(c)

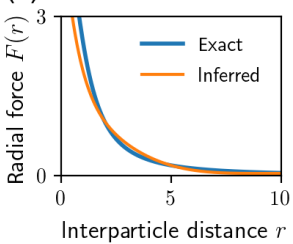

FIG. 7. Stochastic force inference for harmonically trapped active Brownian particles with soft repulsive interactions $F(r)=$ $1 /\left(1+r^{2}\right)$ between particles at distance $r$. (a) Snapshot of a configuration for 25 active particles. The black dots indicate the direction of self-propulsion. We perform SFI on a trajectory of only 25 frames blurred to mimic measurement noise. Background shows the trajectory of one particle and force on each particle, inferred (blue arrows) and exact (black arrows). The fitting basis for SFI consists of a combination of harmonic trapping, constantvelocity self-propulsion, and radial interactions between particles with the form $r^{k} e^{-r / r_{0}}$ with $k=0, \ldots, 5$ and $r_{0}$ a typical nearestneighbor distance between particles. (b) Inferred versus exact components of the force on all particles along the trajectory. (c) Inferred radial force between interacting particles, compared to the exact force.

first approximation, it is rarely strictly the case: For instance, the mobility of colloids depends on their distance to walls and other colloids due to hydrodynamic interactions [47]. In order to mathematically describe Brownian dynamics in the presence of an inhomogeneous diffusion tensor $D_{\mu \nu}(\mathbf{x})$, Eq. (1) should be modified into

$$
\dot{x}_{\mu}=\Phi_{\mu}(\mathbf{x})+\sqrt{2 D(\mathbf{x})}_{\mu \nu} \xi_{\nu}
$$

written in the Itô convention, i.e., evaluating $\mathbf{D}(\mathbf{x})$ at the start of the step. Here, $\Phi_{\mu}$ is the drift, which relates to the physical force through

$$
\Phi_{\mu}(\mathbf{x})=F_{\mu}(\mathbf{x})+\partial_{\nu} D_{\mu \nu}(\mathbf{x})
$$

The additional term $\partial_{\nu} D_{\mu \nu}$, sometimes called "spurious force," combines with the noise term to ensure that the dynamics does not induce currents and probability gradients in the absence of forces [47]. To our knowledge, the only way to infer the physical force is to infer both terms in Eq. (11) independently and involves taking gradients of the inferred diffusion. Here we show how to infer both the diffusion field and the drift field, following the same idea as in Sec. III.

We propose to approximate $D_{\mu \nu}(\mathbf{x})$ by its projection as a linear combination of known functions $D_{\mu \nu}(\mathbf{x}) \approx$ $D_{\mu \nu \alpha} c_{\alpha}(\mathbf{x})$ with $D_{\mu \nu \alpha}=\int D_{\mu \nu}(\mathbf{x}) c_{\alpha}(\mathbf{x}) P(\mathbf{x}) d \mathbf{x}$. As before,

we can estimate the projectors $\hat{c}_{\alpha}$ using trajectory averages; the only missing ingredient is a local estimate $\hat{d}_{\mu \nu}\left(t_{i}\right)$ for the diffusion tensor $D_{\mu \nu}\left(\mathbf{x}\left(t_{i}\right)\right)$. Such an estimator can be constructed as $\hat{d}_{\mu \nu}\left(t_{i}\right)=\Delta x_{\mu}\left(t_{i}\right) \Delta x_{\nu}\left(t_{i}\right) / 2 \Delta t$, so that our estimator for $D_{\mu \nu \alpha}$ reads

$$
\hat{D}_{\mu \nu \alpha}=\frac{1}{\tau} \sum_{i} \hat{d}_{\mu \nu}\left(t_{i}\right) \hat{c}_{\alpha}\left(\mathbf{x}\left(t_{i}\right)\right) \Delta t .
$$

The relative error on these projection coefficients is of order $\sqrt{N_{b} \Delta t / \tau}$ (see Appendix G). Similar to Eq. (7) for the force field, Eq. (12) corresponds to a linear regression of $\hat{d}_{\mu \nu}\left(t_{i}\right)$, and was previously suggested for one-dimensional systems in Ref. [29]. We test this estimator using two minimal models: a one-dimensional ratchet process with sinusoidal force and diffusion coefficient inspired by the Büttiker-Landauer model [48,49] [Figs. 8(d)] and a twodimensional process in a harmonic trap with a constant diffusion gradient [Figs. 8(h)]. We quantitatively recover the diffusion coefficient as a function of the position [Figs. 8(b) and 8(f)] and confirm that the error vanishes in the limit of long trajectories [Figs. 8(d) and 8(h)]. Importantly, the estimator introduced in Eq. (12) is biased in the presence of noise on the measured $\mathbf{x}$ and becomes effectively useless if this noise is larger than the typical $\Delta \mathbf{x}$. Inspired by the estimator proposed by Vestergaard et al. [50] for homogeneous, isotropic diffusion, we define a bias-corrected local estimator

$$
\hat{\mathbf{d}}\left(t_{i}\right)=\frac{\left[\Delta \mathbf{x}\left(t_{i-1}\right)+\Delta \mathbf{x}\left(t_{i}\right)\right]^{2}}{4 \Delta t}+\frac{\Delta \mathbf{x}\left(t_{i}\right) \Delta \mathbf{x}\left(t_{i-1}\right)}{2 \Delta t},
$$

where tensor products are implied. Modifying Eq. (12) accordingly thus corrects measurement noise bias [Fig. 8(h)] at the price of an increased relative error for short trajectories (see Appendix G).

We also approximate the drift as a linear combination of functions $\Phi_{\mu}(\mathbf{x})=\Phi_{\mu \alpha} c_{\alpha}(\mathbf{x})$. Equation (7) provides an estimator for the projection coefficients $\Phi_{\mu \alpha}$ in terms of an Itô integral. This estimator is, however, impractical for experimental data, as even moderate measurement noise induces large errors in these coefficients. As in Eq. (9), we exploit the Itô-to-Stratonovich conversion to obtain an estimator that is not biased by measurement noise:

$$
\hat{\Phi}_{\mu \alpha}=\hat{v}_{\mu \alpha}-\frac{1}{\tau} \sum_{i} \hat{d}_{\mu \nu}\left(t_{i}\right) \partial_{\nu} \hat{c}_{\alpha}\left(\mathbf{x}\left(t_{i}\right)\right) \Delta t
$$

where $\hat{v}_{\mu \alpha}$ is the velocity projection coefficient [Eq. (8)], and $\hat{d}_{\mu \nu}\left(t_{i}\right)$ can either be the local biased-corrected estimator [Eq. (13)] or another estimator of $D_{\mu \nu}\left(\mathbf{x}_{i}\right)$. The convergence properties of $\hat{\Phi}_{\mu \alpha}$ to its asymptotic value are similar to those of Eq. (7). 

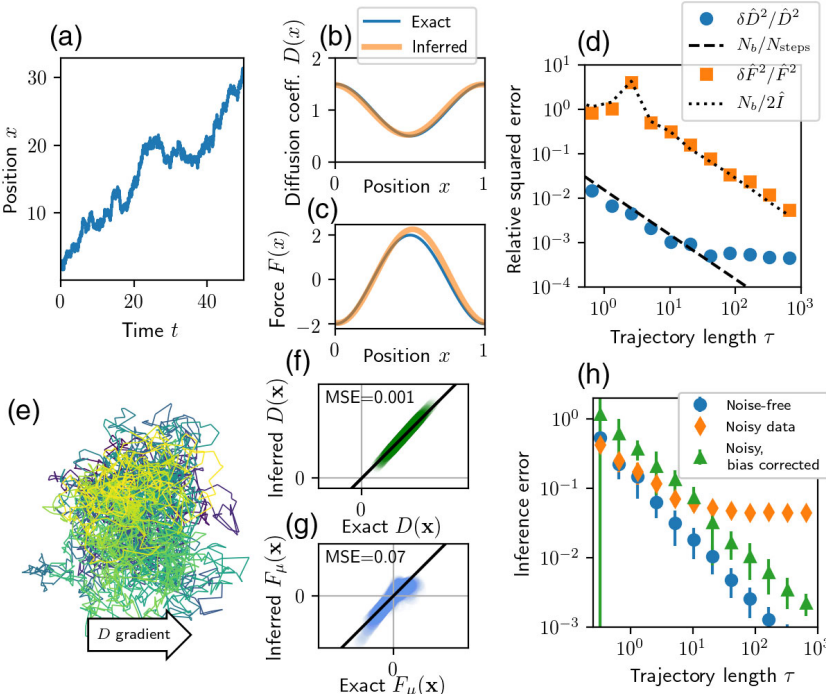

(h)

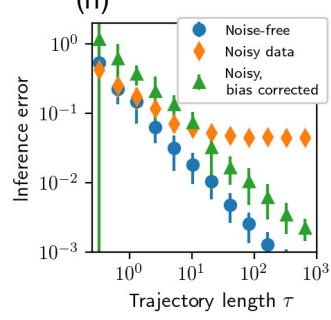

FIG. 8. Stochastic inference of inhomogeneous diffusion and forces. (a) A trajectory of a 1D ratchet model with $F(x)=$ $F_{0} \cos (2 \pi x)$ and $D(x)=1+a \cos (2 \pi x)$, with periodic boundary conditions. (b),(c) For the trajectory presented in (a), inferred and exact diffusion coefficient [using Eq. (12)] and force field [using Eq. (15)] as a function of the position. We use a first-order Fourier basis to infer both force and diffusion. (d) Analysis of the convergence of the diffusion (blue) and force (orange) estimators as a function of the trajectory duration for the process presented in (a). The dotted and dashed black lines are the self-consistent estimates for the squared error, respectively, for the diffusion and the force. The plateau for the diffusion inference is due to the finite time step. (e) A trajectory of a minimal 2D model, an isotropic harmonic trap at equilibrium $F_{\mu}(\mathbf{x})=-D_{\mu \nu}(\mathbf{x}) x_{\nu}$ in a constant gradient of isotropic diffusion $D_{\mu \nu}(\mathbf{x})=\left(1+a_{\rho} x_{\rho}\right) \delta_{\mu \nu}$. (f),(g) Inferred versus exact diffusion coefficient [using Eq. (12)] and force components [using Eq. (15)] along trajectory (a). A linear polynomial basis is used to fit the diffusion coefficient and a quadratic basis to fit $F_{\mu}$. (d) Convergence of the diffusion projection estimator (normalized by the average diffusion tensor) to its exact value for the process shown in (a). Circles, using Eq. (12); diamonds, using Eq. (12) in the presence of timeuncorrelated measurement noise; triangles, using the biascorrected local estimator. Error bars represent the standard deviation over 64 samples. Details and parameters in Appendix H.

We can now combine our diffusion [Eq. (12)] and drift [Eq. (14)] projection estimators to reconstruct the force field,

$$
\hat{F}_{\mu}(\mathbf{x})=\hat{\Phi}_{\mu \alpha} c_{\alpha}(\mathbf{x})-\hat{D}_{\mu \nu \alpha} \partial_{\nu} \hat{c}_{\alpha}(\mathbf{x})
$$

using Eq. (11). This estimator allows for quantitative inference of the force, provided that the divergence of the diffusion coefficient is well approximated. We demonstrate this [Figs. 8(c), 8(d), and 8(g)] for the simple processes presented in Figs. 8(a) and 8(e) using an adapted basis to fit the diffusion coefficient.

\section{DISCUSSION}

In this article, we introduce stochastic force inference, a method to reconstruct force and diffusion fields and measure entropy production from Brownian trajectories. Based on the communication-theory notion of capacity, we show that such trajectories contain a limited amount of information. With finite data, force inference is thus limited by the information available per degree of freedom to infer. SFI uses this information to fit the force field with a linear combination of known functions. We demonstrate its utility on a variety of model systems and benchmark its accuracy using data comparable to current experiments.

We now briefly compare SFI to other existing methods to infer forces from Brownian trajectories. SFI combines the ability to infer arbitrary force fields, for nonequilibrium processes, in high dimensions and in the presence of measurement noise. In contrast, many previous methods essentially rely on a specific linear [51] or parametric [52] form for the force or are specific to one-dimensional systems [29,31,32]. Other approaches include spectral methods [28,53], Bayesian methods [24,25,54,55], maximum-likelihood techniques [21], or methods that rely on coarse graining through constant-by-parts $[23,30,33]$ or linear-by-parts [22] approximations. However, these techniques become inefficient as the system's dimensionality increases. Furthermore, none offers a generic unbiased estimator in the presence of measurement noise. Few of these general methods are being used on experimental data in soft-matter and biological systems. We quantitatively compare SFI to two of the most popular such methods $[23,25,33]$ that rely on spatial binning (Fig. 9). Our method significantly outperforms them for a two-dimensional process simulating single-molecule dynamics in a complex cellular environment, in particular in the presence of realistic measurement noise.

An important by-product of SFI is the ability to quantify the irreversibility of a system by measuring the entropy production associated with its currents. Alternative methods to estimate entropy production also exist, either by coarse graining trajectories to estimate currents $[13,14,17]$, by measuring cycling frequencies $[15,44]$, by using nonMarkovian signatures of irreversibility in hidden variables [45], or by using thermodynamic bounds on the fluctuations of dissipative currents [16,56]. These methods are, however, inherently limited to relatively low-dimensional systems with homogeneous diffusion, and even then require large amounts of well-resolved data. SFI, in contrast, performs well in high dimensions-even with trajectories too short to resolve the steady-state densityand in the presence of measurement noise and inhomogeneous diffusion.

We limit our scope here to systems whose dynamics is described by Eqs. (1) or (10), with a time-independent force field and white-in-time noise. When the force field varies in time, for instance due to the dynamics of unobserved 

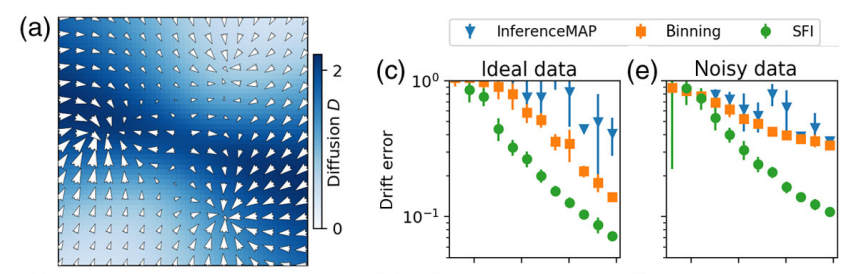

(b)
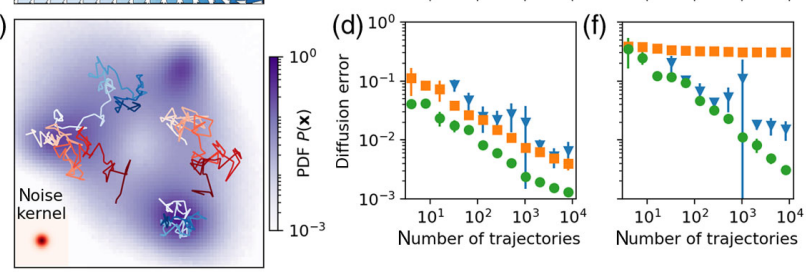

FIG. 9. Quantitative comparison of SFI with other methods on a simulated system mimicking $2 \mathrm{D}$ single-molecule trajectories in a complex cellular environment with multiple potential wells, outof-equilibrium circulation, and space-dependent isotropic diffusion. (a) The diffusion field (blue gradient) and drift field (white arrows, scaled as $|\Phi|^{1 / 2}$ for better legibility). (b) The steady-state probability distribution function (PDF) of the process. The blue traces show two representative trajectories with $n=100$ time steps. The red traces show trajectories blurred by moderate Gaussian measurement error (with amplitude shown as a red kernel). (c)-(f) Comparison of the performance of SFI with adaptive Fourier basis (green circles) and two widely used inference methods: InferenceMAP [25], a Bayesian method for single-molecule inference (blue triangles), and grid-based binning with maximum-likelihood estimation [23,33] [Eq. (7)] and an adaptive mesh size (orange squares). We evaluate the performance of these methods on the approximation of the drift field [(c),(e)] and diffusion field [(d),(f)] as a function of the number $N$ of single-molecule trajectories [similar to those in (b)] used, with ideal data $[(\mathrm{c}),(\mathrm{d})]$ and in the presence of measurement noise [(e), (f)]. The performance is evaluated as the average mean-squared error on the reconstructed field along trajectories. SFI outperforms both other methods in all cases; for noisy data, SFI is the only one that provides an unbiased estimation of the drift. Details and parameters in Appendix $\mathrm{H} 9$.

variables, SFI captures the average projection of the force onto the observed variables (see Appendix E). Furthermore, SFI could be extended to capture an explicit time dependence of the force by using a time-dependent basis. Finally, force inference is notably complicated by non-Markovian terms in the dynamics [57], such as colored noise; however, in such cases, our projection approach to estimate phasespace velocities [Eq. (8)] remains useful and valid.

Our approach, all in all, proposes a solution to the inverse problem of Brownian dynamics: inferring the force and diffusion fields from trajectories. This method consists of a few intelligible equations and provides a powerful data analysis framework that could be used on a broad class of stochastic systems where inferring effective forces and currents from limited noisy data is of interest. Our work thus applies to microscopic systems where thermal noise is relevant, such as single molecules [23], active colloids $[58,59]$, and cytoskeletal filaments $[15,17]$. Beyond thermal systems, for stochastic dynamical systems that can be effectively modeled by Brownian dynamics, applications of our framework range from the behavior of cells $[3,10,60]$ and animals [61] to modeling of climate dynamics [5,51,62] and trend finding in financial data [4]. Our method could be combined with sparsity-promoting techniques, as used to infer dynamical equations in deterministic systems [63], to go from force fitting to identifying the simple rules governing the dynamics.

\section{A. Material and methods}

All formulas presented in this article are derived in the Appendixes, together with the details of each simulated system.

Code availability: A readily usable PYTHON package to perform stochastic force inference is available at [64]. It includes minimal examples.

\section{ACKNOWLEDGMENTS}

The authors warmly thank Eldad Afik, Theo Drivas, Kamesh Krishnamurthy, David Lacoste, Martin Lenz, Ben Machta, Andreas Mayer, Frédéric Van Wijland, and especially Chase Broedersz and his group, for multiple conversations and useful comments. This work was supported in part by the National Science Foundation, through the Center for the Physics of Biological Function (PHY1734030). This work was initiated at the Aspen Center for Physics, which is supported by National Science Foundation Grant No. PHY-1607611. The authors acknowledge support from the Princeton Center for Theoretical Science. P. R. is supported by a Center for the Physics of Biological Function fellowship.

A. F. and P. R. contributed equally to this work.

\section{APPENDIX A: GAUSSIAN CHANNEL INTERPRETATION OF BROWNIAN DYNAMICS}

In this Appendix, we address the question of quantifying the rate at which information can be read out or is encoded in a trajectory. We assume that the system follows the overdamped Langevin equation,

$\dot{x}_{\mu}=F_{\mu}(\mathbf{x})+\sqrt{2 D}_{\mu \nu} \xi_{\nu}, \quad\left\langle\xi_{\mu}(t) \xi_{\nu}\left(t^{\prime}\right)\right\rangle=\delta\left(t-t^{\prime}\right)$.

Here and in the main text, what we refer to as a "force" is in fact the physical force multiplied by the mobility matrix $M$, which has the dimension of a mobility. So, in terms of our $F$, the system is out of equilibrium if $D^{-1} F$ does not derive from a potential. Indeed, a system in equilibrium has a physical force that is derived from a potential, and a mobility matrix which is proportional to the diffusion coefficient: $D=M T$ where $T$ is the temperature. Our approach thus does not distinguish out-of-equilibrium systems due to the difference in the temperature between 
components, such as popular bead-spring models, from systems driven by nonreciprocal force fields. We assume through most of this article that this diffusion matrix is known and space independent (although it can be anisotropic); the case of a spatially variable diffusion matrix and how to infer it from the data is treated in Appendix G. We also assume that a steady state exists and that the system is ergodic, i.e., that time averages converge to phase-space averages. Note, however, that the discussion below can be readily extended to averages over an ensemble of trajectories instead of time averages over a single long trajectory.

The complete force field is characterized by an infinite number of degrees of freedom, and thus, in principle, contains an infinite amount of information (the value of the force components at each location in phase space). It is therefore pertinent to ask if there is a bound to the rate at which this information can be read off from the trajectory. We consider an infinite-length trajectory, from which, in principle, all information about the force field can be recovered. We argue that indeed there is such a maximal rate, given by the capacity (in natural information units, or nats)

$$
C=\frac{1}{4} D_{\mu \nu}^{-1} \int F_{\mu}(\mathbf{x}) F_{\nu}(\mathbf{x}) P(\mathbf{x}) d \mathbf{x} .
$$

To explain this formula, let us first focus on a onedimensional system. A trajectory which satisfies the dynamics given by Eq. (A1) encodes the information about the force field in the form of a continuous-time signal $F(\mathbf{x}(t))$ corresponding to the values of the force field at the points $x(t)$ that the trajectory visits. However, what can actually be read out from the trajectory is $\dot{x}$, i.e., the signal $F(\mathbf{x}(t))$ with noise $\xi$ added to it (Fig. 2). Thus, we can think of the dynamics Eq. (A1) as a noisy communication channel, with Gaussian white-correlated noise, where the information about the force is transmitted in the form of a code word $F(\mathbf{x}(t))$ which satisfies $\lim _{\tau \rightarrow \infty} 1 / \tau \int_{0}^{\tau} F^{2} d t=$ $\int F^{2}(x) P(x) d x$. In communication theory, such a channel is called an infinite-bandwidth Gaussian channel [34]. It has a well-defined capacity, i.e., a maximal rate of information transmission: For code words of duration $\tau$ that satisfy the so-called "power constraint" $1 / \tau \int_{0}^{\tau} d t F^{2}(t) \leq \mathcal{P}$ and a white noise with amplitude $2 D$, the capacity is given by $\mathcal{P} /(4 D)$ nats per second. Information cannot be transmitted through the channel at a faster rate. Stated differently, the capacity quantifies the (exponential) rate with which the maximal number of distinguishable signals grows with the amount of time the channel is used for, in particular as $\tau \rightarrow \infty$. In our case, the capacity is related to the distinguishability of different force fields with the same power constraint. The maximal rate is obtained for a signal which saturates the power constraint so that the relevant constraint to consider is $\mathcal{P}=\lim _{\tau \rightarrow \infty} 1 / \tau \int_{0}^{\tau} F^{2} d t$. Thus, our trajectory which has $\lim _{\tau \rightarrow \infty} 1 / \tau \int_{0}^{\tau} F^{2} d t=\int F^{2} P(x) d x$ cannot produce information about the force field at a rate faster than the capacity as defined in Eq. (A2). Note that in contrast to the usual communication-theory setting, we do not control the code word through which the force field is encoded, only the decoding scheme - the code word is determined by the dynamics, the force field being sampled according to the probability density function (PDF) $P(x)$. To go from the capacity for a one-dimensional process to that of a $d$-dimensional process [Eq. (A2)], we decompose the channel into $d$ parallel channels and add their capacities. Indeed, let us first go into the basis where the noise is diagonal and normalize its amplitude to two, such that all components of the new force $D_{\mu \nu}^{-1 / 2} F_{\nu}$ have the same units $\left(t^{-1 / 2}\right)$. The components of the noise become independent, and the $d$ components in that basis become parallel channels, with signals measured in the same units, whose capacities sum up to Eq. (A2).

\section{The Shannon-Hartley formula and infinite-bandwidth channels}

The infinite-bandwidth capacity of Brownian dynamics, as presented in Eq. (A2), corresponds to that of the continuous dynamics. It can also be seen as the $\Delta t \rightarrow 0$ limit of a discrete signal (i.e., a finite-bandwidth signal) such as can be acquired in practice. The capacity of such a discrete Gaussian channel is given by the Shannon-Hartley formula [34]

$$
C=\frac{1}{2 \Delta t} \log \left(1+\frac{\mathcal{P} \Delta t}{\mathcal{N}}\right),
$$

where we consider as before power-limited signals, where $\mathcal{P} \Delta t / \mathcal{N}$ is the signal-to-noise ratio, $\mathcal{P}$ is the signal power (note that it is not the power of the system in the energetic sense, only in the signal theory sense), and $\mathcal{N} / \Delta t$ the noise power. When the bandwidth is taken to infinity, i.e., $\Delta t \rightarrow 0$, we get

$$
C_{0}=\frac{\mathcal{P}}{2 \mathcal{N}} \log _{2} e \text { bits per sec, }
$$

which corresponds to Eq. (A2). For a finite but small $\Delta t$, the expression for the capacity becomes

$$
C=\frac{\mathcal{P}}{2 \mathcal{N}}-\frac{\mathcal{P}^{2} \Delta t}{4 \mathcal{N}^{2}}+\cdots \approx C_{0}\left(1-C_{0} \Delta t\right) .
$$

The first correction to the continuous-time capacity due to the finite rate of sampling is thus of relative order $C_{0} \Delta t$, i.e., the information per sample. The loss of information when monitoring Brownian dynamics at a finite rate is thus negligible provided that the information per sample remains small. This has an important practical consequence for experimental applications, where there is often a tradeoff between the acquisition rate and duration of the 
experiment (for instance, due to photobleaching of fluorescent proteins): When the information per sample becomes small, very little can be learned about the force field by increasing the acquisition frequency.

\section{APPENDIX B: INFORMATION AT THE TRAJECTORY LEVEL}

In this Appendix, we relate the notion of capacity to trajectory-level quantities and relate it to other stochastic thermodynamics quantities: the entropy production and the inflow rate. While Appendix A is restricted to the case of constant-diffusion Brownian dynamics, here we consider the general case with not only a state-dependent force, but also a state-dependent diffusion tensor. In that case, the noise is no longer additive: It has a multiplicative component, and care must be taken to specify the convention within which the Langevin equation is written. We use the Itô convention here, writing

$$
\dot{x}_{\mu}=\Phi_{\mu}(\mathbf{x})+\sqrt{2 D(\mathbf{x})}_{\mu \nu} \xi_{\nu},
$$

where $\Phi_{\mu}(\mathbf{x})=F_{\mu}(\mathbf{x})+\partial_{\nu} D_{\mu \nu}(\mathbf{x})$ is the drift term [47], and $F_{\mu}(\mathbf{x})$ equals the mobility matrix times the physical force.

\section{The capacity as a Kullback-Leibler divergence rate}

To relate the capacity to path-dependent quantities, we consider a trajectory $\mathcal{C}^{N}=\{\mathbf{x}(0), \mathbf{x}(\Delta t), \ldots, \mathbf{x}(N \Delta t)\}$, with $t_{i}=i \Delta t$, and where we define the discrete difference $\Delta x_{\mu}\left(t_{i}\right)=x_{\mu}\left(t_{i}+\Delta t\right)-x_{\mu}\left(t_{i}\right)$ and $\tau=N \Delta t$. The path integral formula for the probability density $\mathcal{P}\left(\mathcal{C}^{N} \mid F\right)$ of a trajectory $\mathcal{C}^{N}$ in the force field $F$ written in the Itô convention reads [39]

$$
\begin{aligned}
\mathcal{P}\left(\mathcal{C}^{N} \mid F\right) & =\frac{P_{0}(\mathbf{x}(0))}{(4 \pi)^{d N / 2}} \prod_{i=0}^{N-1} \frac{1}{\left\{\operatorname{det} D\left(\mathbf{x}\left(t_{i}\right)\right) \Delta t\right\}^{1 / 2}} \\
& \times \exp \left[-\frac{1}{4} \Delta t\left(\frac{\Delta x_{\mu}\left(t_{i}\right)}{\Delta t}-F_{\mu}\left(\mathbf{x}\left(t_{i}\right)\right)-\partial_{\rho} D_{\mu \rho}\left(\mathbf{x}\left(t_{i}\right)\right)\right)\right. \\
& \left.\times D_{\mu \nu}^{-1}\left(\mathbf{x}\left(t_{i}\right)\right)\left(\frac{\Delta x_{\mu}\left(t_{i}\right)}{\Delta t}-F_{\nu}\left(\mathbf{x}\left(t_{i}\right)\right)-\partial_{\sigma} D_{\nu \sigma}\left(\mathbf{x}\left(t_{i}\right)\right)\right)\right] .
\end{aligned}
$$

Note that in the limit of long trajectories, the initial point probability becomes unimportant. We show here that the capacity of the system relates to the Kullback-Leibler divergence rate between $\mathcal{P}\left(\mathcal{C}^{N} \mid F\right)$ and the probability density at zero force (but with the same diffusion field), $\mathcal{P}\left(\mathcal{C}^{N} \mid 0\right) \equiv \mathcal{P}\left(\mathcal{C}^{N} \mid F=0\right):$

$$
\begin{aligned}
C & =\lim _{\tau \rightarrow \infty} \frac{1}{\tau} \int \mathcal{D} \mathcal{C}^{\tau} \mathcal{P}\left(\mathcal{C}^{\tau} \mid F\right) \log \frac{\mathcal{P}\left(\mathcal{C}^{\tau} \mid F\right)}{\mathcal{P}\left(\mathcal{C}^{\tau} \mid 0\right)} \\
& =\left\langle\frac{1}{4} F_{\mu}(\mathbf{x}(t)) D_{\mu \nu}^{-1}(\mathbf{x}(t)) F_{\nu}(\mathbf{x}(t))\right\rangle .
\end{aligned}
$$

Indeed, for a constant diffusion coefficient, the right-hand side of the above equation reduces to the capacity discussed in Appendix A 2, Eq. (A2). Note that for systems with multiplicative noise, to the best of our knowledge, a formula for the channel capacity, as defined in transmission theory, has yet to be derived. Moreover, the interpretation from the standpoint of transmission theory is further complicated as, from physical considerations, we wish to infer $F_{\mu}$ rather than $\Phi_{\mu}$. However, one may use the trajectory-based formula in Eq. (B4) as a general definition of the capacity for Brownian dynamics. Then, the generalization of Eq. (A2) to systems with inhomogeneous diffusion is seen to be

$$
C=\frac{1}{4} \int D_{\mu \nu}^{-1}(\mathbf{x}) F_{\mu}(\mathbf{x}) F_{\nu}(\mathbf{x}) P(\mathbf{x}) d \mathbf{x} .
$$

Let us proceed to show Eq. (B4),

$$
\begin{aligned}
C= & \lim _{\tau \rightarrow \infty} \frac{1}{\tau} \int \mathcal{D} \mathcal{C}^{\tau} \mathcal{P}\left(\mathcal{C}^{\tau} \mid F\right) \log \frac{\mathcal{P}\left(\mathcal{C}^{\tau} \mid F\right)}{\mathcal{P}\left(\mathcal{C}^{\tau} \mid 0\right)} \\
= & \lim _{\tau \rightarrow \infty} \frac{1}{\tau}\left\langle\frac{1}{2} \int^{\mathrm{I} t \hat{}} d t \dot{x}_{\mu} D_{\mu \nu}^{-1} F_{\nu}(\mathbf{x}(t))\right. \\
& -\frac{1}{2} \int_{0}^{\tau} d t\left(\partial_{\rho} D_{\rho \mu}\right) D_{\mu \nu}^{-1} F_{\nu}(\mathbf{x}(t)) \\
& \left.-\frac{1}{4} \int_{0}^{\tau} d t F_{\mu} D_{\mu \nu}^{-1} F_{\nu}(\mathbf{x}(t))\right\rangle \\
= & \left\langle\frac{1}{4} F_{\mu}(\mathbf{x}(t)) D_{\mu \nu}^{-1}(\mathbf{x}(t)) F_{\nu}(\mathbf{x}(t))\right\rangle,
\end{aligned}
$$

where we use that $\left\langle\int^{\text {Itô }} d t \dot{x}_{\mu} D_{\mu \nu}^{-1} F_{\nu}(\mathbf{x}(t))\right\rangle=$ $\left\langle\int_{0}^{\tau} d t\left(F_{\mu}+\partial_{\rho} D_{\rho \mu}\right) D_{\mu \nu}^{-1} F_{\nu}(\mathbf{x}(t))\right\rangle$. Note that passing between the first and second line in the above equation is equivalent to deriving the Girsanov formula for diffusions.

\section{The inflow rate}

In the main text, we connect the capacity to the inflow rate $G=\int d \mathbf{x} P(\mathbf{x}) g_{\mu} D_{\mu \nu} g_{\nu}$ with $g_{\mu}=\partial_{\mu} \log P$. This quantity was originally introduced and studied by Baiesi and Falasco [36] in the case of Brownian dynamics with homogeneous diffusion (and for discrete Markov processes, not discussed here). We generalize it here to systems with inhomogeneous diffusion and discuss its properties. 


\section{a. Relation between the inflow rate and an instantaneous entropy production rate}

Let us show that it corresponds to an instantaneous entropy production rate that would be present if the force was suddenly set to zero. Consider the entropy $S(t)=-\int d \mathbf{x} P(x, t) \log P(x, t)$, after the force is set to zero: $F_{\mu}=0$, denoting that instant by $t=0$. At that instant, one has $\partial_{t} P=\partial_{\mu}\left[D_{\mu \nu} \partial_{\nu} P\right]$. Then,

$$
\begin{aligned}
\left.\partial_{t} S\right|_{t=0}= & -\int d \mathbf{x} \log P(\mathbf{x}) \partial_{\mu}\left[D_{\mu \nu} \partial_{\nu} P(\mathbf{x})\right] \\
& +\int d \mathbf{x} \partial_{\mu}\left(D_{\mu \nu} \partial_{\nu} P\right) \\
= & \int d \mathbf{x} \frac{\partial_{\mu} P(\mathbf{x})}{P} D_{\mu \nu} \partial_{\nu} P(\mathbf{x}) \\
= & \int d \mathbf{x} P(\mathbf{x}) \partial_{\mu} \log P(\mathbf{x}) D_{\mu \nu}(\mathbf{x}) \partial_{\nu} \log P(\mathbf{x}) \\
= & G,
\end{aligned}
$$

where we use integration by parts, assuming the boundary terms vanish. We can define $v_{\mu}^{\text {Fick }}=-D_{\mu \nu} g_{\nu}$, a Fick velocity related to the current $j_{\mu}^{\text {Fick }}=-D_{\mu \nu} \partial_{\nu} P$, that would result from diffusion of particles with an initial density profile $P(\mathbf{x})$ in the absence of forces. Indeed, in these notations, $G$ has a similar form to the entropy production rate

$$
G=\int v_{\mu}^{\text {Fick }} v_{\nu}^{\text {Fick }} D_{\mu \nu}^{-1} P(\mathbf{x}) d \mathbf{x} .
$$

However, the inflow rate is nonzero even at equilibrium. It measures the heterogeneity of the steady-state probability distribution. Indeed, for an equilibrium process $F^{\mu}=$ $D_{\mu \nu} \partial_{\mu} \log P$ (and $G=C$ trivially). In a sense, it is the amount of information that the force field needs to continuously inject into the system in order to maintain its spatial structure, while the entropy production can be seen as the amount of information the force field injects into the system to maintain its currents.

\section{b. The inflow rate as a phase-space contraction rate}

The relation $D_{\mu \nu} g_{\mu}=F_{\mu}-v_{\mu}$ (which holds for a spacedependent diffusion tensor) can be used to rewrite the inflow rate as

$$
\begin{aligned}
G & =\int d \mathbf{x} P(\mathbf{x}) g_{\mu} D_{\mu \nu} g_{\nu}=\int d \mathbf{x} P(\mathbf{x})\left(\partial_{\mu} \log P\right)\left(F_{\mu}-v_{\mu}\right) \\
& =\int d \mathbf{x}\left[\partial_{\mu} P(\mathbf{x})\right] F_{\mu}+\int d \mathbf{x} \partial_{\mu}\left[v_{\mu} P(\mathbf{x})\right] \log P \\
& =-\int d \mathbf{x} P(\mathbf{x}) \partial_{\mu} F_{\mu},
\end{aligned}
$$

where in the second line the steady-state relation $\partial_{\mu}\left[v_{\mu} P(\mathbf{x})\right]=\partial_{\mu} j_{\mu}=0$ is employed. We thus obtain an expression for the inflow rate as (minus) the average divergence of the force. In a deterministic dynamical system, this is equal to the average sum of the Lyapunov exponents and is called the average phase-space contraction rate. It then corresponds to the mean rate of entropy production in the environment [38]. For nondeterministic systems, it was mentioned in Ref. [38] as a "natural entropy production." It is worth stressing the difference between the deterministic case and overdamped Brownian dynamics in this context. While for a deterministic system at equilibrium, i.e., a Hamiltonian system, the divergence of the force is identically zero due to the symplectic structure (there is no entropy production), for an equilibrium overdamped system whose divergence is nonzero. Indeed, the inflow rate (which does not correspond to an actual entropy production in this case) is positive, as we discuss above.

\section{c. Trajectory-based interpretation of the inflow rate}

Here we prove that an equivalent expression for the inflow rate is

$$
\begin{aligned}
G & =\lim _{\tau \rightarrow \infty} \frac{1}{\tau} \int \mathcal{D} \mathcal{C}^{\tau} \mathcal{P}\left(\mathcal{C}^{\tau} \mid F\right) \log \frac{\mathcal{P}\left(\mathcal{C}^{\tau} \mid F\right)}{\mathcal{P}\left(-\mathcal{C}^{\tau} \mid-F\right)} \\
& =\lim _{\tau \rightarrow \infty} \frac{1}{\tau}\left\langle\log \frac{\mathcal{P}\left(\mathcal{C}^{\tau} \mid F\right)}{\mathcal{P}\left(-\mathcal{C}^{\tau} \mid-F\right)}\right\rangle_{F}
\end{aligned}
$$

The simplest way to do that is to express the probability density of a trajectory [Eq. (B3)] in an alternative form, as we now show. We begin with the expression for the probability of a transition to the point $\mathbf{x}$ from the point $\mathbf{x}^{\prime}$ in an infinitesimal time $\Delta t$ [39],

$$
\begin{aligned}
P\left(\mathbf{x}, t+\Delta t \mid \mathbf{x}^{\prime}, t\right)= & \frac{1}{\sqrt{(4 \pi)^{d} \operatorname{det} D(\mathbf{x}) \Delta t}} \exp \left[\Delta t \left\{-\partial_{\mu} \Phi_{\mu}(\mathbf{x})+\partial_{\mu} \partial_{\nu} D_{\mu \nu}(\mathbf{x})\right.\right. \\
& \left.\left.-\frac{1}{4}\left(\frac{x_{\mu}-x_{\mu}^{\prime}}{\Delta t}-\Phi_{\mu}(\mathbf{x})+2 \partial_{\rho} D_{\mu \rho}(\mathbf{x})\right) D_{\mu \nu}^{-1}(\mathbf{x})\left(\frac{x_{\nu}-x_{\nu}^{\prime}}{\Delta t}-\Phi_{\nu}(\mathbf{x})+2 \partial_{\sigma} D_{\nu \sigma}(\mathbf{x})\right)\right\}\right] .
\end{aligned}
$$

Note that here the diffusion coefficient and $\Phi_{\mu}$ are both evaluated at the point $\mathbf{x}$ to which the system transitions. The probability of a trajectory is then simply given by a product of such transition probabilities and the distribution of the initial point. Using that $\Phi_{\mu}=F_{\mu}+\partial_{\nu} D_{\mu \nu}$, we then get 


$$
\begin{aligned}
\mathcal{P}\left(\mathcal{C}^{N} \mid F\right)= & \frac{P_{0}(\mathbf{x}(0))}{(4 \pi)^{d N / 2}} \prod_{i=0}^{N-1} \frac{1}{\left(\operatorname{det} D\left(\mathbf{x}\left(t_{i+1}\right)\right) \Delta t\right)^{1 / 2}} \exp \left[-\partial_{\mu} F_{\mu}\left(\mathbf{x}\left(t_{i+1}\right)\right) \Delta t-\frac{1}{4} \Delta t\left(\frac{\Delta x_{\mu}\left(t_{i}\right)}{\Delta t}-F_{\mu}\left(\mathbf{x}\left(t_{i+1}\right)\right)+\partial_{\rho} D_{\mu \rho}\left(\mathbf{x}\left(t_{i+1}\right)\right)\right)\right. \\
& \left.\times D_{\mu \nu}^{-1}\left(\mathbf{x}\left(t_{i+1}\right)\right)\left(\frac{\Delta x_{\nu}\left(t_{i}\right)}{\Delta t}-F_{\nu}\left(\mathbf{x}\left(t_{i+1}\right)\right)+\partial_{\sigma} D_{\nu \sigma}\left(\mathbf{x}\left(t_{i+1}\right)\right)\right)\right] .
\end{aligned}
$$

It follows that the probability of the time-reversed trajectory $-\mathcal{C}^{N}=\left\{\mathbf{x}\left(t_{N}\right), \mathbf{x}\left(t_{N-1}\right), \ldots, \mathbf{x}\left(t_{0}\right)\right\}$ can be written in the form

$$
\begin{aligned}
\mathcal{P}\left(-\mathcal{C}^{N} \mid F\right)= & \frac{P_{0}(\mathbf{x}(N \Delta t))}{(4 \pi)^{d N / 2}} \prod_{i=0}^{N-1} \frac{1}{\left\{\operatorname{det} D\left(\mathbf{x}\left(t_{i}\right)\right) \Delta t\right\}^{1 / 2}} \exp \left[-\partial_{\mu} F_{\mu}\left(\mathbf{x}\left(t_{i}\right)\right) \Delta t-\frac{1}{4} \Delta t\left(\frac{-\Delta x_{\mu}\left(t_{i}\right)}{\Delta t}-F_{\mu}\left(\mathbf{x}\left(t_{i}\right)\right)+\partial_{\rho} D_{\mu \rho}\left(\mathbf{x}\left(t_{i}\right)\right)\right)\right. \\
& \left.\times D_{\mu \nu}^{-1}\left(\mathbf{x}\left(t_{i}\right)\right)\left(\frac{-\Delta x_{\nu}\left(t_{i}\right)}{\Delta t}-F_{\nu}\left(\mathbf{x}\left(t_{i}\right)\right)+\partial_{\sigma} D_{\nu \sigma}\left(\mathbf{x}\left(t_{i}\right)\right)\right)\right] .
\end{aligned}
$$

Now, it becomes straightforward to evaluate Eq. (B12), dividing term by term in the product in Eq. (B3) by the product in $\mathcal{P}\left(-\mathcal{C}^{N} \mid-F\right)$ using Eq. (B15) with the reversed sign for the force. Indeed, we notice that all terms cancel out except for the divergence of $F_{\mu}$, which yields (we ignore the terms related to the initial and final distributions whose contribution vanishes in the limit of $\tau \rightarrow \infty$ )

$$
\begin{aligned}
G & =\lim _{\tau \rightarrow \infty} \frac{1}{\tau} \int \mathcal{D} \mathcal{C}^{\tau} \mathcal{P}\left(\mathcal{C}^{\tau} \mid F\right) \log \frac{\mathcal{P}\left(\mathcal{C}^{\tau} \mid F\right)}{\mathcal{P}\left(-\mathcal{C}^{\tau} \mid-F\right)} \\
& =-\lim _{\tau \rightarrow \infty} \int_{0}^{\tau} \frac{d t}{\tau}\left\langle\partial_{\mu} F_{\mu}(\mathbf{x}(t))\right\rangle .
\end{aligned}
$$

\section{Different decompositions of the capacity and the relation to traffic}

The trajectory-based expression for the capacity Eq. (B4) is related to the "dynamical entropy" introduced in
Ref. [37]: It is equal to the dynamical entropy per unit time in the limit $\tau \rightarrow \infty$, i.e., to a rate of dynamical entropy. In Ref. [37], the dynamical entropy was split into two contributions: a time-anti-symmetric contribution equal to $\dot{S} / 2$ and a time-symmetric contribution $-\mathcal{T}$, where $\mathcal{T}$ is called the traffic (and is related to the so-called frenesy in Markov jump processes). The relations between the capacity, the inflow rate we define, the entropy production, and the steady-state traffic $\mathcal{T}$ are

$$
C=-\mathcal{T}+\frac{1}{2} \dot{S}, \quad \mathcal{T}=(\dot{S}-G) / 4
$$

The decomposition of the capacity that we present in the main text can also be presented as the sum of timesymmetric and -anti-symmetric parts but corresponding to a different trajectory-based expression for the capacity:

$$
\begin{aligned}
4 C & =\lim _{\tau \rightarrow \infty} \frac{1}{\tau} \int \mathcal{D} \mathcal{C}^{\tau} \mathcal{P}\left(\mathcal{C}^{\tau} \mid F\right) \log \frac{\mathcal{P}\left(\mathcal{C}^{\tau} \mid F\right)}{\mathcal{P}\left(\mathcal{C}^{\tau} \mid-F\right)}=\lim _{\tau \rightarrow \infty} \frac{1}{\tau}\left\langle\log \frac{\mathcal{P}\left(\mathcal{C}^{\tau} \mid F\right)}{\mathcal{P}\left(\mathcal{C}^{\tau} \mid-F\right)}\right\rangle_{F} \\
& =\lim _{\tau \rightarrow \infty} \frac{1}{\tau}\left\langle\int^{\text {Itô }} d t \dot{x}_{\mu} D_{\mu \nu}^{-1} F_{\nu}(\mathbf{x}(t))-\int_{0}^{\tau} d t\left(\partial_{\rho} D_{\rho \mu}\right) D_{\mu \nu}^{-1} F_{\nu}(\mathbf{x}(t))\right\rangle \\
& =\lim _{\tau \rightarrow \infty} \frac{1}{\tau}\left\langle\int{ }^{\text {Strat }} d t \dot{x}_{\mu} D_{\mu \nu}^{-1} F_{\nu}(\mathbf{x}(t))\right\rangle-\lim _{\tau \rightarrow \infty} \frac{1}{\tau}\left\langle\left\langle\int_{0}^{\tau} d t D_{\mu \rho} \partial_{\rho}\left(D_{\mu \nu}^{-1} F_{\nu}\right)(\mathbf{x}(t))-\int_{0}^{\tau} d t \partial_{\rho}\left(D_{\rho \mu}\right) D_{\mu \nu}^{-1} F_{\nu}(\mathbf{x}(t))\right\rangle\right. \\
& =\lim _{\tau \rightarrow \infty} \frac{1}{\tau}\langle\underbrace{\int_{\text {Strat }} d t \dot{x}_{\mu} D_{\mu \nu}^{-1} F_{\nu}(\mathbf{x}(t))}_{\text {time antisymmetric }}\rangle+\lim _{\tau \rightarrow \infty} \frac{1}{\tau}\langle\underbrace{-\int_{0}^{\tau} d t \partial_{\mu} F_{\mu}(\mathbf{x}(t))}_{\text {time symmetric }}\rangle-\lim _{\tau \rightarrow \infty} \frac{1}{\tau}\langle\int_{0}^{\tau} d t F_{\nu} \underbrace{\partial_{\rho}\left(D_{\rho \mu} D_{\mu \nu}^{-1}\right)}_{0}\rangle .
\end{aligned}
$$

Indeed, the first term in the last line is time antisymmetric and is equal to the entropy production rate, and the second term is time symmetric and is equal to the inflow rate.
One can think of the decomposition of the capacity into $\dot{S}$ and $G$ as decomposing the influence of the force field into two types of "orders": "go there" corresponding to a dissipative, irreversible motion quantified by $\dot{S}$ and "stay 
there" corresponding to a nondissipative, reversible motion fighting thermal diffusion and quantified by $G$.

\section{APPENDIX C: STOCHASTIC FORCE INFERENCE: ESTIMATING $F_{\mu \alpha}$ AND ITS ERROR}

In this Appendix, we derive the core results of our article: how to perform SFI in practice and self-consistently estimate the error in the inference.

\section{The force as a trajectory average}

To be able to deduce the force from the trajectory, one first needs an expression for the force in terms of measurable quantities along the trajectory. We have

$$
\begin{aligned}
\mathbf{F}(\mathbf{x}) & =\lim _{\epsilon \rightarrow 0}\left\langle\frac{(\mathbf{x}(t+\epsilon)-\mathbf{x}(t))}{\epsilon} \mid \mathbf{x}(t)=\mathbf{x}\right\rangle \\
& =\left\langle\dot{\mathbf{x}}^{+} \mid \mathbf{x}(t)\right\rangle=\left\langle\delta(\mathbf{x}(t)-\mathbf{x}) \dot{\mathbf{x}}^{+}\right\rangle / P(x),
\end{aligned}
$$

where $\langle\cdot \mid \mathbf{x}(t)=\mathbf{x}\rangle$ means averaging over realizations of the noise, conditioned on being at position $\mathbf{x}$ at time $t$. We define here $\dot{\mathbf{x}}^{+}$as the right-hand derivative corresponding to Itô calculus (see Appendix A of Ref. [40]). The coefficients of the force field in its decomposition with respect to the phase-space projector $c_{\alpha}(\mathbf{x})$ are

$$
\begin{aligned}
F_{\mu \alpha} & =\int d \mathbf{x} P(\mathbf{x}) F_{\mu}(\mathbf{x}) c_{\alpha}(\mathbf{x})=\int d \mathbf{x}\left\langle\delta(\mathbf{x}(t)-\mathbf{x}) \dot{x}_{\mu}^{+}\right\rangle c_{\alpha}(\mathbf{x}) \\
& =\left\langle\int d \mathbf{x} \delta(\mathbf{x}(t)-\mathbf{x}) \dot{x}_{\mu}^{+} c_{\alpha}(\mathbf{x})\right\rangle=\left\langle\dot{x}_{\mu}^{+} c_{\alpha}(\mathbf{x})\right\rangle .
\end{aligned}
$$

Because of this last expression, the force projection coefficient $F_{\mu \alpha}$ can be expressed as an average quantity along an infinitely long trajectory, which can thus be estimated by computing it on a finite trajectory.

Note that, similar to the force, the phase-space velocity can also be defined through an average of $\dot{\mathbf{x}}$, where the time derivative is taken in the Stratonovich sense:

$$
\begin{aligned}
\mathbf{v}(\mathbf{x}) & =\lim _{\epsilon \rightarrow 0}\left\langle\frac{(\mathbf{x}(t+\epsilon)-\mathbf{x}(t-\epsilon))}{2 \epsilon} \mid \mathbf{x}(t)=\mathbf{x}\right\rangle \\
& =\left\langle\frac{1}{2}\left(\dot{\mathbf{x}}^{+}+\dot{\mathbf{x}}^{-}\right) \mid \mathbf{x}(t)=\mathbf{x}\right\rangle \\
& =\left\langle\delta(\mathbf{x}(t)-\mathbf{x}) \frac{1}{2}\left(\dot{\mathbf{x}}^{+}+\dot{\mathbf{x}}^{-}\right)\right\rangle / P(\mathbf{x})
\end{aligned}
$$

(see Appendix A of Ref. [40]). The phase-space velocity in its decomposition with respect to the phase-space basis $c_{\alpha}(\mathbf{x})$ is analogous to the force

$$
v_{\mu \alpha}=\left\langle\frac{1}{2}\left(\dot{x}_{\mu}^{+}+\dot{x}_{\mu}^{-}\right) c_{\alpha}(\mathbf{x})\right\rangle .
$$

\section{Projection on the empirical basis}

The second difficulty in evaluating Eq. (2) of the main text in practice is that the phase-space measure $P(\mathbf{x})$ is unknown in practice. As a consequence, the phase-space basis $c_{\alpha}(\mathbf{x})$ is not known either, as it is the orthonormalized basis derived from $b$ using $P$ as the measure. Our approach consists of approximating $P(\mathbf{x})$ by the empirical measure

$$
\hat{P}_{\tau}(\mathbf{x})=\frac{1}{\tau} \int_{0}^{\tau} \delta(\mathbf{x}-\mathbf{x}(t)) d t
$$

corresponding to a time average along the trajectory.

We then define the empirical projector $\hat{c}_{\alpha}$ with respect to this measure, as in the main text:

$\hat{c}_{\alpha}(\mathbf{x})=\hat{B}_{\alpha \beta}^{-1 / 2} b_{\beta}(\mathbf{x})$ with $\hat{B}_{\alpha \beta}=\int b_{\alpha}(\mathbf{x}) b_{\beta}(\mathbf{x}) \frac{d t}{\tau}$.

In the long-trajectory limit, these "empirical projectors" $\hat{c}_{\alpha}(\mathbf{x})$ converge to the phase-space projectors $c_{\alpha}(\mathbf{x})$; more precisely, we expect that for typical trajectories $\hat{c}_{\alpha}(\mathbf{x})=c_{\alpha}(\mathbf{x})+O\left(\sqrt{\tau_{0} / \tau}\right)$, where $\tau$ is the duration of the trajectory and $\tau_{0}$ is a relaxation time of the system. In the case of the polynomial basis, for instance, the convergence of the basis at order $n$ is related to the convergence of the $n$th cumulant of the probability distribution function. We do not seek to make this statement more mathematically precise here.

As an intermediate variable for this calculation, we define the projection coefficients $F_{\mu \alpha}^{\tau}$ of the (exact) force onto these empirical projectors. These coefficients are trajectory dependent; however, $\hat{c}_{\alpha}$ are directly accessible from the trajectory, as is the empirical measure with respect to which they are projectors, so that obtaining the coefficients $F_{\mu \alpha}^{\tau}$ precisely would result in an accurate approximation of the force field $F_{\mu} \approx F_{\mu \alpha}^{\tau} \hat{c}_{\alpha}$ along the trajectory. For this reason, we focus here on how the estimator $\hat{F}_{\mu \alpha}$ as defined in Eq. (6) of the main text converges to $F_{\mu \alpha}^{\tau}$. The relative errors presented in the main text also refer to this convergence (rather than the convergence to the phasespace projection $F_{\mu \alpha}$ ). Recall that our estimator is given by

$$
\begin{aligned}
\hat{F}_{\mu \alpha} & =\frac{1}{\tau} \int^{\mathrm{It} \hat{\mathrm{o}}} \hat{c}_{\alpha}(\mathbf{x}) d \mathbf{x}_{t}^{\mu} \\
& =\underbrace{\frac{1}{\tau} \int_{0}^{\tau} \hat{c}_{\alpha}(\mathbf{x}) F_{\mu}(\mathbf{x}) d t}_{F_{\mu \alpha}^{\tau}}+\underbrace{\frac{1}{\tau} \int^{\mathrm{It} \hat{o}} \hat{c}_{\alpha}(\mathbf{x}) \sqrt{2} D_{\mu \nu}^{1 / 2} d \xi_{t}^{\nu}}_{Z_{\mu \alpha}}
\end{aligned}
$$

using the Langevin equation (A1). Since $F_{\mu \alpha}^{\tau}$ is what we wish to infer, we propose to study now the statistics of $Z_{\mu \alpha}=\hat{F}_{\mu \alpha}-F_{\mu \alpha}^{\tau}$, i.e., its mean and variance. 


\section{Statistics of the error in the inference of the projection coefficients}

We thus study the first and second moment of the random tensor $Z_{\mu \alpha}$, i.e., respectively, the systematic bias and the typical error of $\hat{F}_{\mu \alpha}$ as an estimator of $F_{\mu \alpha}^{\tau}$. To make the norm of these moments meaningful, it is necessary here to go to dimensionless coordinates: Indeed, different phasespace coordinates can have different dimensions (such as, for instance, a phase space comprising both distances and angles, as in Fig. 7 of the main text), and thus different coordinates of $Z_{\mu \alpha}$ cannot be compared or summed. To this end, we define $W_{\mu \alpha}=D_{\mu \nu}^{-1 / 2} Z_{\nu \alpha}$, all the coordinates of which have the dimension of $t^{-1 / 2}$.

First, recall that we define both phase-space and empirical projectors as a linear combination of the basis functions $b, c_{\alpha}=B_{\alpha \beta}^{-1 / 2} b_{\beta}$, and $\hat{c}_{\alpha}=\hat{B}_{\alpha \beta}^{-1 / 2} b_{\beta}$, where

$$
\begin{aligned}
B_{\alpha \beta} & =\int d \mathbf{x} P(\mathbf{x}) b_{\beta}(\mathbf{x}) b_{\alpha}(\mathbf{x}), \\
\hat{B}_{\alpha \beta} & =\int_{0}^{\tau} \frac{d t}{\tau} b_{\beta}(\mathbf{x}(t)) b_{\alpha}(\mathbf{x}(t)) .
\end{aligned}
$$

Thus, we have $\lim _{\tau \rightarrow \infty} \hat{B}_{\alpha \beta}^{-1 / 2}=B_{\alpha \beta}^{-1 / 2}$ and $\left\langle\hat{B}_{\alpha \beta}\right\rangle=B_{\alpha \beta}$. Let us denote $\Delta_{\alpha \beta}=B_{\alpha \gamma}^{1 / 2} \hat{B}_{\gamma \beta}^{-1 / 2}-\delta_{\alpha \beta}$ the dimensionless error on the orthonormalization matrix (indeed, the basis functions $b_{\alpha}$ can, in principle, have a dimension). We have $\lim _{\tau \rightarrow \infty} \Delta_{\alpha \beta}=0$; typically, we will have more precisely $\Delta_{\alpha \beta}=O(1 / \sqrt{\tau})$ corresponding to the convergence of trajectory integrals to phase-space integrals in Eq. (C9). We then have

$$
\begin{aligned}
Z_{\mu \alpha} \equiv & \frac{1}{\tau} \int^{\mathrm{It} \hat{o}} \hat{c}_{\alpha}(\mathbf{x}) \sqrt{2} D_{\mu \nu}^{1 / 2} d \xi_{t}^{\nu} \\
= & B_{\alpha \beta}^{-1 / 2} \sqrt{2} D_{\mu \nu}^{1 / 2} \frac{1}{\tau} \int^{\mathrm{Itô}} b_{\beta}(\mathbf{x}) d \xi_{t}^{\nu} \\
& +B_{\alpha \beta}^{-1 / 2} \Delta_{\beta \gamma} \sqrt{2} D_{\mu \nu}^{1 / 2} \frac{1}{\tau} \int^{\mathrm{It} \hat{0}} b_{\gamma}(\mathbf{x}) d \xi_{t}^{\nu} .
\end{aligned}
$$

For the remainder of this section, we denote the Itô integral by a regular integration: $\int^{\text {Itô }} d \xi_{t}^{\nu}=\int_{0}^{\tau} d \xi_{t}^{\nu}$. We now put an upper bound on the first moment of $Z_{\mu \alpha}$, i.e., on the systematic bias. Note that the first term in Eq. (C10) has zero average, as it is linear in the noise. In contrast, due to possible correlations between the noise and the random variable $\Delta_{\alpha \beta}$, the second term may not average to zero. Going to dimensionless coordinates, we use the CauchySchwarz inequality to bound the norm of this bias:

$$
\begin{aligned}
\left\|\left\langle W_{\mu \alpha}\right\rangle\right\|^{2} & =\left\|\left\langle B_{\alpha \beta}^{-1 / 2} \Delta_{\beta \gamma} \frac{1}{\tau} \int_{0}^{\tau} b_{\gamma}(\mathbf{x}) D_{\mu \nu}^{-1 / 2} \sqrt{2} D_{\nu \rho}^{1 / 2} d \xi_{t}^{\rho}\right\rangle\right\|^{2} \\
& \leq 2 B_{\beta \delta}^{-1}\left\langle\Delta_{\beta \rho} \Delta_{\rho \delta}\right\rangle\left\langle\frac{1}{\tau^{2}} \int_{0}^{\tau} b_{\gamma}(\mathbf{x}) d \xi_{t}^{\mu} \int_{0}^{\tau} b_{\gamma}(\mathbf{x}) d \xi_{t^{\prime}}^{\mu}\right\rangle .
\end{aligned}
$$

We can then use the Itô isometry relation [65] to prove that

$$
\begin{aligned}
& \left\langle\int_{0}^{\tau} b_{\alpha}(\mathbf{x}) d \xi_{t}^{\mu} \int_{0}^{\tau} b_{\beta}(\mathbf{x}) d \xi_{t^{\prime}}^{\mu}\right\rangle \\
& =\left\langle\int_{0}^{\tau} b_{\alpha}(\mathbf{x}(t)) b_{\beta}(\mathbf{x}(t)) d t\right\rangle=\left\langle\hat{B}_{\alpha \beta}\right\rangle,
\end{aligned}
$$

which implies that

$$
\left\|\left\langle W_{\mu \alpha}\right\rangle\right\|^{2} \leq \frac{2}{\tau} B_{\beta \delta}^{-1}\left\langle\Delta_{\beta \rho} \Delta_{\rho \delta}\right\rangle\left\langle\hat{B}_{\gamma \gamma}\right\rangle .
$$

Since $\Delta_{\alpha \beta}=O\left(\tau^{-1 / 2}\right)$, we thus have $\left\langle W_{\mu \alpha}\right\rangle=O(1 / \tau)$, which corresponds to a fast convergence of the bias toward zero: The bias is negligible compared to the fluctuating part of inference error, which goes as $O\left(\tau^{-1 / 2}\right)$.

Indeed, let us now compute the second moment of $W_{\mu \alpha}$. We have

$$
\begin{aligned}
& \left\langle W_{\mu \alpha} W_{\nu \beta}\right\rangle \\
& \quad=\frac{2}{\tau^{2}}\left\langle\hat{B}_{\alpha \gamma}^{-1 / 2} \hat{B}_{\beta \delta}^{-1 / 2} \int_{0}^{\tau} \int_{0}^{\tau} d \xi_{t}^{\mu} d \xi_{t^{\prime}}^{\nu} b_{\gamma}(\mathbf{x}(t)) b_{\delta}\left(\mathbf{x}\left(t^{\prime}\right)\right)\right\rangle .
\end{aligned}
$$

As $\hat{B}_{\alpha \gamma}^{-1 / 2}$ depends on all values of $t$, it is not adapted to the Wiener process $d \xi_{t}^{\mu}$, and thus, we cannot apply the Itô isometry. However, we have $\hat{B}_{\alpha \gamma}^{-1 / 2}=B_{\alpha \beta}^{-1 / 2}\left(\delta_{\beta \gamma}+\Delta_{\beta \gamma}\right)$. Applying the Itô isometry [Eq. (C12)] yields

$$
\begin{aligned}
\left\langle W_{\mu \alpha} W_{\nu \beta}\right\rangle & =\frac{1}{\tau^{2}} \delta_{\mu \nu} B_{\alpha \gamma}^{-1 / 2} B_{\beta \delta}^{-1 / 2} 2 \tau\left\langle\hat{B}_{\gamma \delta}\right\rangle+R_{\mu \alpha \nu \beta} \\
& =\frac{2}{\tau} \delta_{\mu \nu} \delta_{\alpha \beta}+R_{\mu \alpha \nu \beta}
\end{aligned}
$$

where we define the remainder

$$
\begin{aligned}
R_{\mu \alpha \nu \beta}= & \frac{2}{\tau^{2}}\left\langle\left(B_{\alpha \gamma}^{-1 / 2} B_{\beta \lambda}^{-1 / 2} \Delta_{\lambda \delta}+B_{\alpha \lambda}^{-1 / 2} \Delta_{\lambda \gamma} \hat{B}_{\beta \delta}^{-1 / 2}\right)\right. \\
& \left.\times \int_{0}^{\tau} \int_{0}^{\tau} d \xi_{t}^{\mu} d \xi_{t^{\prime}}^{\nu} b_{\gamma}(\mathbf{x}(t)) b_{\delta}\left(\mathbf{x}\left(t^{\prime}\right)\right)\right\rangle,
\end{aligned}
$$

which is, as we show now, subleading in Eq. (C15). We now wish to bound the amplitude of the remainder $\left|\left\langle W_{\mu \alpha} W_{\mu \alpha}\right\rangle-(2 / \tau) N_{b}\right|=\left|R_{\mu \alpha \mu \alpha}\right|$. Since for typical trajectories $\Delta_{\alpha \beta}=O\left(\tau^{-1 / 2}\right)$, we can bound every element of the matrix $\left|B_{\alpha \gamma}^{-1 / 2} B_{\alpha \lambda}^{-1 / 2} \Delta_{\lambda \delta}+B_{\alpha \lambda}^{-1 / 2} \Delta_{\lambda \gamma} \hat{B}_{\alpha \delta}^{-1 / 2}\right| \leq R O_{\gamma \delta}$ for such trajectories, where $R=O(1 / \sqrt{\tau})$ is a (nonfluctuating) number, and $O_{\gamma \delta}$ is the matrix with 1's at all places. We get 


$$
\begin{aligned}
\left|\left\langle W_{\mu \alpha} W_{\mu \alpha}\right\rangle-\frac{2}{\tau} N_{b}\right| & =\frac{2}{\tau^{2}}\left|\left\langle\left(B_{\alpha \gamma}^{-1 / 2} B_{\alpha \lambda}^{-1 / 2} \Delta_{\lambda \delta}+B_{\alpha \lambda}^{-1 / 2} \Delta_{\lambda \gamma} \hat{B}_{\alpha \delta}^{-1 / 2}\right) \int_{0}^{\tau} \int_{0}^{\tau} d \xi_{t}^{\mu} d \xi_{t^{\prime}}^{\mu} b_{\gamma}(\mathbf{x}(t)) b_{\delta}\left(\mathbf{x}\left(t^{\prime}\right)\right)\right\rangle\right| \mid \\
& \leq \frac{2}{\tau^{2}}\left\langle\left|\left(B_{\alpha \gamma}^{-1 / 2} B_{\alpha \lambda}^{-1 / 2} \Delta_{\lambda \delta}+B_{\alpha \lambda}^{-1 / 2} \Delta_{\lambda \gamma} \hat{B}_{\alpha \delta}^{-1 / 2}\right)\right| \int_{0}^{\tau} \int_{0}^{\tau} d \xi_{t}^{\mu} d \xi_{t^{\prime}} b_{\gamma}(\mathbf{x}(t)) b_{\delta}\left(\mathbf{x}\left(t^{\prime}\right)\right) \mid\right\rangle \\
& \leq \frac{2}{\tau^{2}} R O_{\gamma \delta}\left\langle\left|\int_{0}^{\tau} \int_{0}^{\tau} d \xi_{t}^{\mu} d \xi_{t^{\prime}}^{\mu} b_{\gamma}(\mathbf{x}(t)) b_{\delta}\left(\mathbf{x}\left(t^{\prime}\right)\right)\right|\right\rangle \\
& \leq \frac{2}{\tau^{2}} R O_{\gamma \gamma}\left\langle\left|\int_{0}^{\tau} \int_{0}^{\tau} d \xi_{t}^{\mu} d \xi_{t^{\prime}}^{\mu} b_{\delta}(\mathbf{x}(t)) b_{\delta}\left(\mathbf{x}\left(t^{\prime}\right)\right)\right|\right\rangle \\
& =\frac{2}{\tau^{2}} R O_{\gamma \gamma}\left\langle\int_{0}^{\tau} d \xi_{t}^{\mu} b_{\delta}(\mathbf{x}(t)) \int_{0}^{\tau} d \xi_{t}^{\mu} b_{\delta}(\mathbf{x}(t))\right\rangle=\frac{1}{\tau^{2}} R O_{\gamma \gamma} 2 \tau\left\langle\hat{B}_{\delta \delta}\right\rangle=O\left(1 / \tau^{3 / 2}\right) .
\end{aligned}
$$

In the fourth line, we use that for two semidefinite matrices $M_{\alpha \beta}$ and $N_{\alpha \beta}, M_{\alpha \beta} N_{\beta \alpha} \leq \sqrt{M_{\alpha \alpha}^{2} N_{\beta \beta}^{2}} \leq M_{\alpha \alpha} N_{\beta \beta}$, an identity based on the Cauchy-Schwarz inequality. In the fifth line, we employ the Itô isometry [Eq. (C12)]. Again, this subleading term originates from the convergence of the empirical projected basis to its long-trajectory limit.

\section{Self-consistent estimate of the error on the projected force}

The previous error estimates are rigorous but require knowledge of the exact force field to assess their amplitude. The goal of this section is to provide approximate estimates of the typical error that can be obtained using only the inferred force field and are thus useful in practical situations. Now that we know the statistical properties of the dimensionless error term $W_{\mu \alpha}$, we can write the covariance of the inferred force projection coefficients explicitly:

$\left\langle\left(\hat{F}_{\mu \alpha}-F_{\mu \alpha}^{\tau}\right)\left(\hat{F}_{\nu \alpha}-F_{\nu \alpha}^{\tau}\right)\right\rangle=\frac{2 D_{\mu \nu}}{\tau} \delta_{\alpha \beta}[1+O(1 / \sqrt{\tau})]$.

Now, let us define the information along the trajectory by

$$
I_{b}^{\tau}=\frac{1}{4} \tau F_{\mu \alpha}^{\tau} D_{\mu \nu}^{-1} F_{\nu \alpha}^{\tau}
$$

In the long time limit, the rate of information $I_{b}^{\tau} / \tau$ converges to the capacity we discussed previously. Similarly, we define the empirical estimate of the information along the trajectory,

$$
\begin{aligned}
\hat{I}_{b} & =\frac{\tau}{4} \hat{F}_{\mu \alpha} D_{\mu \nu}^{-1} \hat{F}_{\nu \alpha}=I_{b}^{\tau}+\frac{1}{2} \tau F_{\mu \alpha}^{\tau} D_{\mu \nu}^{-1} Z_{\nu \alpha}+\frac{1}{4} \tau Z_{\mu \alpha} D_{\mu \nu}^{-1} Z_{\nu \alpha} \\
& =I_{b}^{\tau}+\frac{1}{2} \tau \hat{F}_{\mu \alpha} D_{\mu \nu}^{-1} Z_{\nu \alpha}-\frac{1}{4} \tau Z_{\mu \alpha} D_{\mu \nu}^{-1} Z_{\nu \alpha}
\end{aligned}
$$

so that

$$
I_{b}^{\tau}=\hat{I}_{b}-\frac{1}{2} \tau \hat{F}_{\mu \alpha} D_{\mu \nu}^{-1} Z_{\nu \alpha}+\frac{1}{4} \tau Z_{\mu \alpha} D_{\mu \nu}^{-1} Z_{\nu \alpha}
$$

We can also relate the average of the empirical information to the trajectory information:

$$
\left\langle\hat{I}_{b}\right\rangle-I_{b}^{\tau}=\frac{1}{2} N_{b}
$$

at leading order. The estimator $\hat{I}_{b}$ is thus biased, with bias $\frac{1}{2} N_{b}$. The variance of this estimator is well approximated by $\left\langle\left(I_{b}^{\tau}-\hat{I}_{b}\right)^{2}\right\rangle \approx 2\left\langle\hat{I}_{b}\right\rangle+N_{b}^{2} / 4$.

In practice, the "true" force field is not known-inferring it is the goal here. It is therefore important to provide an estimate of the inference error using only the inferred quantities. Equation (C19) allows us to propose such a selfconsistent estimate of the error. Indeed, it can be interpreted as the (squared) typical error on the force projection coefficients, and its right-hand side can be estimated using only trajectory-dependent quantities (again, we assume that the diffusion matrix is known). We can also combine these quantities in a single number quantifying the relative inference error as

$$
\frac{\left(F_{\mu \alpha}^{\tau}-\hat{F}_{\mu \alpha}\right) D_{\mu \nu}^{-1}\left(F_{\nu \alpha}^{\tau}-\hat{F}_{\nu \alpha}\right)}{\hat{F}_{\mu \alpha} D_{\mu \nu}^{-1} \hat{F}_{\nu \alpha}} \sim N_{b} / 2 \hat{I} .
$$

Thus, $N_{b} / 2 \hat{I}$ provides a self-consistent estimate of the relative error. Note that in the absence of forces, $\langle\hat{I}\rangle=$ $N_{b} / 2$, corresponding to an inferred error of 1 , which is consistent. Similarly, based on our estimate of the variance of $\hat{I}_{b}$, we define a self-consistent confidence interval around this inferred information as $\delta \hat{I}_{b}^{2}=2 \hat{I}_{b}+N_{b}^{2} / 4$.

\section{The force estimator and maximum likelihood}

Here we show that the estimator we propose in Eq. (C8) is also the maximum-log-likelihood estimator for $F_{\mu \alpha}$. Indeed, given a measured trajectory $C^{\tau}$, we use the expression for the probability of a trajectory Eq. (B3) to calculate 
$0=\frac{\partial \log \mathcal{P}\left(\mathcal{C}^{\tau} \mid F\right)}{\partial F_{\mu \alpha}^{\tau}}=\int d \mathbf{x} \frac{\partial \log \mathcal{P}\left(\mathcal{C}^{\tau} \mid F\right)}{\partial F_{\nu}(\mathbf{x})} \frac{\partial F_{\nu}(\mathbf{x})}{\partial F_{\mu \alpha}^{\tau}}$.

We have

$\frac{\partial \log \mathcal{P}\left(\mathcal{C}^{\tau} \mid F\right)}{\partial F_{\nu}(\mathbf{x})}=\frac{1}{2} \int_{0}^{\tau} d t D_{\nu \mu}^{-1}\left\{\dot{x}_{\mu}(t)-F_{\mu}(\mathbf{x}(t))\right\} \delta(\mathbf{x}-\mathbf{x}(t))$.

Next, the empirical projectors $\hat{c}_{\alpha}$ corresponding to the trajectory give the decomposition of the force as

$$
F_{\nu}(\mathbf{x})=F_{\nu \alpha}^{\tau} \hat{c}_{\alpha}(\mathbf{x})+F_{\nu}^{\perp}
$$

so that

$$
\frac{\partial F_{\nu}(\mathbf{x})}{\partial F_{\mu \alpha}^{\tau}}=\hat{c}_{\alpha}(\mathbf{x}) \delta_{\mu \nu}
$$

and

$$
\begin{aligned}
0 & =\int d \mathbf{x} \frac{\partial \log \mathcal{P}\left(\mathcal{C}^{\tau} \mid F\right)}{\partial F_{\nu}(\mathbf{x})} \frac{\partial F_{\nu}(\mathbf{x})}{\partial F_{\mu \alpha}^{\tau}} \\
& =\int d \mathbf{x} \hat{c}_{\alpha}(\mathbf{x}) \int_{0}^{\tau} d t\left\{\dot{x}_{\nu}(t)-F_{\nu}(\mathbf{x}(t))\right\} \delta(\mathbf{x}-\mathbf{x}(t))
\end{aligned}
$$

resulting in

$$
\begin{aligned}
& \int_{0}^{\tau} d t \dot{x}_{\nu}(t) \hat{c}_{\alpha}(\mathbf{x}(t)) \underbrace{\int d \mathbf{x} \delta(\mathbf{x}-\mathbf{x}(t))}_{1} \\
& =\int d \mathbf{x} \hat{c}_{\alpha}(\mathbf{x}) F_{\nu}(\mathbf{x}) \underbrace{\int_{0}^{\tau} d t \delta(\mathbf{x}-\mathbf{x}(t))}_{\tau \hat{P}(\mathbf{x})}=\tau F_{\nu \alpha}^{\tau},
\end{aligned}
$$

which is solved by our estimator in Eq. (C8). This estimator indeed maximizes the log-likelihood, since $\hat{c}_{\alpha}(\mathbf{x})$ is independent of $F_{\mu \alpha}^{\tau}$ so that

$$
\begin{aligned}
\frac{\partial \log \mathcal{P}\left(\mathcal{C}^{\tau} \mid F\right)}{\partial F_{\mu \alpha}^{\tau} \partial F_{\nu \beta}^{\tau}} & =\frac{\partial}{\partial F_{\nu \beta}^{\tau}} \int d \mathbf{x} \frac{1}{2} \int_{0}^{\tau} d t D_{\mu \rho}^{-1}\left\{\dot{x}_{\rho}(t)-F_{\rho}(\mathbf{x}(t))\right\} \delta(\mathbf{x}-\mathbf{x}(t)) \hat{c}_{\alpha}(\mathbf{x}) \\
& =-\int d \mathbf{x} \frac{1}{2} \int_{0}^{\tau} d t D_{\mu \nu}^{-1} \delta(\mathbf{x}-\mathbf{x}(t)) \hat{c}_{\alpha}(\mathbf{x}(t)) \hat{c}_{\beta}(\mathbf{x}(t))=-\frac{\tau}{2} \delta_{\alpha \beta} D_{\mu \nu}^{-1},
\end{aligned}
$$

which is a negative definite matrix.

\section{APPENDIX D: INFERENCE OF VELOCITIES AND ENTROPY PRODUCTION}

In this Appendix, we show how our approach allows the inference of entropy production and phase-space currents (or more specifically, phase-space velocities). We start by some phase-space reminders about the entropy production and then discuss how to infer the entropy produced from a given trajectory.

\section{Phase-space entropy production}

The steady-state entropy production rate is defined via [19]

$$
\begin{aligned}
\dot{S} & =\int \mathrm{d} \mathbf{x} P(\mathbf{x}) v_{\mu}(\mathbf{x}) D_{\mu \nu}^{-1} v_{\nu}(\mathbf{x}) \\
& =\int \mathrm{d} \mathbf{x} P(\mathbf{x}) v_{\mu}(\mathbf{x}) D_{\mu \nu}^{-1} F_{\nu}(\mathbf{x}),
\end{aligned}
$$

where $v_{\nu}(\mathbf{x})=j_{\nu}(\mathbf{x}) / P(x)$ is the phase-space velocity explicitly given by

$$
v_{\mu}=F_{\mu}-D_{\mu \nu} \partial_{\nu} \log P(\mathbf{x}),
$$

and $j_{\nu}$ is the phase-space current. The equality between the two expressions for the entropy production arises from the steady-state condition $\partial_{\mu} j_{\mu}=0$, implying that $g_{\mu}=$ $\partial_{\nu} \log P(\mathbf{x})$ is orthogonal to $v_{\mu}$ with respect to the phase-space measure.

The quantity $\int d \mathbf{x} P(\mathbf{x}) v_{\mu}(\mathbf{x}) D_{\mu \nu}^{-1} F_{\nu}(\mathbf{x})$ is the entropy production related to the heat produced in the bath. Indeed, if the Einstein relation between the mobility and diffusion matrix holds, then this term corresponds to the average work performed by the force divided by the temperature. As the system is overdamped, any work performed is dissipated into heat. Note, however, that even if we do not assume the Einstein relation holds (i.e., that the origin of the white noise is a heat bath), this quantity is related to time irreversibility.

\section{Entropy production along a trajectory}

One can define the entropy production along the trajectory, or equivalently, the dissipated heat divided by the temperature corresponding to the work performed by the force as [19]

$$
\Delta \Pi^{\tau}=\int^{\text {Strat }} \mathbf{D}^{-1} \mathbf{F} \cdot \dot{\mathbf{x}} \mathrm{d} t=\int_{0}^{\tau} D_{\mu \nu}^{-1} F_{\nu}(\mathbf{x}(t)) \circ d x_{\mu}^{t},
$$


where the integral is to be understood in the Stratonovich sense (which, following usual notations, we denote as $\circ d x_{\mu}^{t}$ ). This entropy production is often referred to as the entropy produced in the medium, and one can also define what is called the total entropy production along the trajectory (medium + system) [19]. Assuming the initial point is drawn from the steady-state PDF, the total entropy production is given by

$$
\Delta S^{\tau}=\int_{0}^{\tau} D_{\mu \nu}^{-1} v^{\nu}(\mathbf{x}(t)) \circ d x_{\mu}^{t} .
$$

In the limit $\tau \rightarrow \infty$, when divided by $\tau$, the two definitions for the entropy production converge to the same limit, equal to the entropy production rate in the system $\dot{S}=$ $\int D_{\mu \nu}^{-1} v_{\nu}(\mathbf{x}) v_{\mu}(\mathbf{x}) P(\mathbf{x}) d \mathbf{x}=\int D_{\mu \nu}^{-1} F_{\nu}(\mathbf{x}) v_{\mu}(\mathbf{x}) P(\mathbf{x}) d \mathbf{x}$.

\section{Velocity and entropy production inference}

The probability density $P(\mathbf{x})$ is generally not accessible so that the phase-space velocity cannot be directly computed. However, we have already discussed the empirical density $\hat{P}(\mathbf{x})$, and we can also define the empirical current (see, e.g., Ref. [66])

$$
\begin{aligned}
\hat{j}_{\mu}(\mathbf{x})= & \frac{1}{\tau} \int_{0}^{\tau} \delta(\mathbf{x}(t)-\mathbf{x}) \circ d x_{\mu}^{t} \\
= & F_{\mu}(\mathbf{x}) \hat{P}(\mathbf{x})-D_{\mu \nu} \partial_{\nu} \hat{P}(\mathbf{x}) \\
& +\frac{1}{\tau} \int^{\mathrm{It} \hat{o}} \delta(\mathbf{x}(t)-\mathbf{x}) d \xi_{\mu}^{t}
\end{aligned}
$$

using in the last line that $\mathbf{x}(t)$ satisfies the Langevin equation and the relation between Itô and Stratonovich integrals. This motivates the definition for the empirical phase-space velocity

$$
\hat{v}_{\mu}=\hat{j}_{\mu}(\mathbf{x}) / \hat{P}(\mathbf{x}),
$$

and allows us to write

$$
\begin{aligned}
\Delta \Pi^{\tau} & =\tau \int F_{\mu}(\mathbf{x}) D_{\mu \nu}^{-1} \hat{j}_{\nu}(\mathbf{x}) d \mathbf{x} \\
& =\tau \int F_{\mu}(\mathbf{x}) D_{\mu \nu}^{-1} \hat{v}_{\nu}(\mathbf{x}) \hat{P}(\mathbf{x}) d \mathbf{x} .
\end{aligned}
$$

Note that in this last equation, the force is the exact force, but the velocity (and probability measure) is the empirical one defined in Eq. (D6) so that we obtain the trajectorywise entropy production related to the heat as in Eq. (D3). If we now insert into this relation the projection onto the empirical basis of the force and phase-space velocity, we get the entropy production corresponding to that basis:

$$
\Delta \Pi_{b}^{\tau}=\tau F_{\mu \alpha}^{\tau} D_{\mu \nu}^{-1} \hat{v}_{\nu \alpha}
$$

where

$$
\begin{aligned}
\hat{v}_{\mu \alpha} & =\int^{\mathrm{Strat}} \dot{x}_{\mu} \hat{c}_{\alpha}(\mathbf{x}) \frac{d t}{\tau} \\
& =F_{\mu \alpha}^{\tau}+D_{\mu \nu} \int \partial_{\nu} \hat{c}_{\alpha}(\mathbf{x}) \frac{d t}{\tau}+\frac{1}{\tau} \int^{\mathrm{I} \hat{\mathrm{o}}} \hat{c}_{\alpha}(\mathbf{x}) \sqrt{2} D_{\mu \nu}^{1 / 2} d \xi_{\nu}^{t} \\
& =\hat{F}_{\mu \alpha}+D_{\mu \nu} \int \partial_{\nu} \hat{c}_{\alpha}(\mathbf{x}) \frac{d t}{\tau}
\end{aligned}
$$

using integration by parts. The estimator for the entropy production related to the basis is

$$
\Delta \hat{\Pi}_{b}=\tau \hat{F}_{\mu \alpha} D_{\mu \nu}^{-1} \hat{v}_{\nu \alpha}=\Delta \Pi_{b}^{\tau}+\tau Z_{\mu \alpha} D_{\mu \nu}^{-1} \hat{v}_{\nu \alpha} .
$$

It is important to note that the projected entropy production corresponding to the heat is not positive definite unless we are able to resolve the entire force. Therefore, it does not give a bound on the entropy produced. Furthermore, recall that as is the case for the projection onto the phase-space basis, the projected total entropy and that related to heat generically differ.

On the other hand, the projection of the total entropy production is positive definite, and therefore, it does give a lower bound on the entropy production. The expression $\hat{v}_{\mu \alpha} D_{\mu \nu}^{-1} \hat{v}_{\nu \alpha}$ may be viewed as an estimator of the projection of the total entropy production $\Delta S_{b}^{\tau} / \tau$ or the total entropy production rate in the steady state $\dot{S}_{b}=v_{\mu \alpha} D_{\mu \nu}^{-1} v_{\mu \alpha}$; however, some caution is required. Indeed, consider

$$
\begin{aligned}
\Delta S^{\tau} / \tau & =\int v_{\mu}(\mathbf{x}) D_{\mu \nu}^{-1} \hat{j}_{\nu}(\mathbf{x}) d \mathbf{x} \\
& =\int v_{\mu}(\mathbf{x}) D_{\mu \nu}^{-1} \hat{v}_{\nu}(\mathbf{x}) \hat{P}(\mathbf{x}) d \mathbf{x}
\end{aligned}
$$

(note that one velocity is empirical and the other is exact in this equation). Then,

$$
\Delta S_{b}^{\tau} / \tau=v_{\mu \alpha}^{\tau} D_{\mu \nu}^{-1} \hat{v}_{\nu \alpha}
$$

and we can define the estimator

$$
\hat{\dot{S}}_{b}=\hat{v}_{\mu \alpha} D_{\mu \nu}^{-1} \hat{v}_{\nu \alpha}
$$

This estimator is less controlled than the estimator we have for $\dot{\Pi}_{b}^{\tau}$. Indeed, the estimator $\hat{v}$ has two sources of error as an estimator of $v$. Defining

$$
\tilde{v}_{\mu}=F_{\mu}-D_{\mu \nu} \partial_{\nu} \log \hat{P}(\mathbf{x})
$$

with the empirical PDF rather than the actual one, we have $\hat{v}=\tilde{v}_{\mu}+(1 / \tau) \int^{\mathrm{Ito}} \delta(\mathbf{x}(t)-\mathbf{x}) \sqrt{2} D_{\mu \nu}^{1 / 2} d \xi_{t}^{\nu} / \hat{P}(\mathbf{x})$. In particular, for the projection onto the empirical basis 


$$
\hat{v}_{\mu \alpha}=\tilde{v}_{\mu \alpha}^{\tau}+Z_{\mu \alpha},
$$

where $\tilde{v}_{\mu \alpha}^{\tau}-v_{\mu \alpha}^{\tau}=\delta v_{\mu \alpha}^{\tau} \neq 0, v_{\mu \alpha}^{\tau}$ being the projection of the actual phase-space velocity onto the empirical basis. This is in contrast to the force, where our estimator includes the projection of the actual force.

We write

$$
\begin{aligned}
\hat{\dot{S}} & =\Delta \hat{S}_{b} / \tau=\hat{v}_{\mu \alpha} D_{\mu \nu}^{-1} \hat{v}_{\nu \alpha} \\
& =\Delta S_{b}^{\tau} / \tau+Z_{\mu \alpha} D_{\mu \nu}^{-1} \hat{v}_{\nu \alpha}+\delta v_{\mu \alpha}^{\tau} D_{\mu \nu}^{-1} \hat{v}_{\nu \alpha} .
\end{aligned}
$$

This is a biased estimator, since $\left\langle Z_{\mu \alpha} D_{\mu \nu}^{-1} \hat{v}_{\nu \alpha}\right\rangle \approx$ $\left\langle Z_{\mu \alpha} D_{\mu \nu}^{-1} Z_{\nu \alpha}\right\rangle=\left(2 N_{b} / \tau\right)$. We do not have a formal estimate for the last term $\delta v_{\mu \alpha}^{\tau} D_{\mu \nu}^{-1} \hat{v}_{\nu \alpha}$, but we expect $\delta v_{\mu \alpha}^{\tau} \sim$ $O(1 / \sqrt{\tau})$ so that a reasonable estimate seems to be

$\hat{\dot{S}}_{b}=\frac{\Delta S_{b}^{\tau}}{\tau}+\frac{2 N_{b}}{\tau}+O\left(\sqrt{\frac{2 \hat{v}_{\mu \alpha} D_{\mu \nu}^{-1} \hat{v}_{\nu \alpha}}{\tau}+\left(\frac{2 N_{b}}{\tau}\right)^{2}}\right)$.

Here, for the estimate of the fluctuating part (the error term), we estimate $\left\langle Z_{\mu \alpha} D_{\mu \nu}^{-1} Z_{\nu \alpha} Z_{\rho \beta} D_{\rho \sigma}^{-1} Z_{\sigma \beta}\right\rangle \sim O\left[\left(2 N_{b} / \tau\right)^{2}\right]$, and the contribution in the square root is the dominant term when $v_{\mu \alpha}^{\tau}$ is nonzero, i.e., there is signal. We focus on the long time limit $\tau \rightarrow \infty, \dot{S}^{\tau} \rightarrow \dot{S}$ so that naturally an estimator of $\Delta S_{b}^{\tau} / \tau$ becomes also an estimator of $\dot{S}_{b}$, with deviations which are again of order $O(1 / \sqrt{\tau})$. Thus, we may finally estimate

$$
\hat{\dot{S}}_{b}=\dot{S}_{b}+\frac{2 N_{b}}{\tau}+O\left(\sqrt{\frac{2 \hat{\hat{S}}_{b}}{\tau}+\left(\frac{2 N_{b}}{\tau}\right)^{2}}\right),
$$

where $\dot{S}_{b}=v_{\mu \alpha} D_{\mu \nu}^{-1} v_{\mu \alpha}$. Note that this is an order-ofmagnitude error estimate, not a fully rigorous one.

\section{APPENDIX E: INCOMPLETE OBSERVATIONS AND TIME-DEPENDENT FORCES}

In this article, we make strong assumptions on the dynamics of the system we observe: that it obeys a Langevin dynamics for the observed degrees of freedom $\mathbf{x}$ and that the force field in phase space is time independent. These two assumptions are linked. Indeed, consider the very relevant case of systems which obey a Langevin dynamics, but for which not all degrees of freedom are observable. In that case, the force on the observed degrees of freedom depends on the state of the hidden variables, therefore, apparently violating the assumptions of our formalism. It is interesting to note, however, that this violation is only superficial. Indeed, "hiding" some degrees of freedom of the system is completely equivalent to using a projection basis where these degrees of freedom do not appear explicitly (i.e., functions that are constant with respect to these degrees of freedom). Therefore, provided that the system as a whole obeys a constant-force Langevin equation, SFI will capture the projection of the dynamics onto the observed degrees of freedom, effectively averaging over the hidden ones. Indeed, assume that the force takes the form $F(\mathbf{x}, \mathbf{y})$ where only $\mathbf{x}$ can be measured. We thus project the force field onto a set of function $b_{\alpha}(\mathbf{x})$ that depends only on $\mathbf{x}$. Hence,

$$
\begin{aligned}
F_{\mu \alpha} & =\int d \mathbf{x} d \mathbf{y} P(\mathbf{x}, \mathbf{y}) c_{\alpha}(\mathbf{x}) F_{\mu}(\mathbf{x}, \mathbf{y}) \\
& =\int d \mathbf{x} d y P(\mathbf{y} \mid \mathbf{x}) F_{\mu}(\mathbf{x}, \mathbf{y}) P(\mathbf{x}) c_{\alpha}(\mathbf{x}) \\
& =\int d \mathbf{x} \bar{F}(x) P(x) c_{\alpha}(\mathbf{x}),
\end{aligned}
$$

where $\bar{F}(\mathbf{x})=\int d y P(\mathbf{y} \mid \mathbf{x}) F_{\mu}(\mathbf{x}, \mathbf{y})$ is the force at $\mathbf{x}$ averaged over $\mathbf{y}$. A similar formula applies to the phase-space velocity, as well as when replacing the phase-space integral by a time integral - in which case, one replaces the phasespace measure with the empirical measure. As a consequence, our formulas for the projected entropy production and capacity remain valid and provide lower bounds to total entropy production and capacity of the system:

$$
\begin{aligned}
\dot{S}_{\mathrm{tot}} & =\int d \mathbf{x} d \mathbf{y} P(\mathbf{x}, \mathbf{y}) v_{\mu}(\mathbf{x}, \mathbf{y}) D_{\mu \nu}^{-1} v_{\nu}(\mathbf{x}, \mathbf{y}) \\
& =\int d \mathbf{x} P(\mathbf{x}) D_{\mu \nu}^{-1} \bar{v}_{\mu} v_{\nu}(\mathbf{x}) \geq \int d \mathbf{x} P(\mathbf{x}) D_{\mu \nu}^{-1} \bar{v}_{\mu}(\mathbf{x}) \bar{v}_{\mu}(\mathbf{x}) \\
& \geq D_{\mu \nu}^{-1} v_{\mu \alpha} v_{\mu \alpha}=\dot{S}_{b},
\end{aligned}
$$

where we apply Jensen's inequality twice.

\section{APPENDIX F: INFERENCE WITH IMPERFECT DATA: MEASUREMENT NOISE AND TIME DISCRETIZATION}

Our inference method relies heavily on computing $\dot{\mathbf{x}}$, i.e., the first time derivative of the signal, and on being able to resolve the difference between Itô and Stratonovich time derivatives for (the white-noise part of) the signal. One expects that measurement noise would then swamp the signal and make the distinction between the two, and thus, our inference method, impractical. It turns out, however, that even in the presence of measurement noise, we can suggest estimators $\hat{v}_{\mu \alpha}$ and $\hat{F}_{\mu \alpha}$, which are unbiased by the measurement noise and accurately capture the currents and forces, respectively.

Indeed, let us consider a noisy measure $\mathbf{y}$ of the system's state $\mathbf{x}$ at discrete times $t_{i}=i \Delta t$ defined as

$$
y_{\mu}\left(t_{i}\right)=x_{\mu}\left(t_{i}\right)+\eta_{\mu}^{i}, \quad\left\langle\eta_{\mu}^{i} \eta_{\nu}^{j}\right\rangle=\Lambda_{\mu \nu} \delta_{i, j},
$$

where $\mathbf{x}$ obeys the dynamics (A1), and $\eta$ is the measurement noise (which we assume to be of zero average, 
without loss of generality). We assume this noise to be uncorrelated between different (discrete) time points. Consider first the estimator $\hat{F}_{\mu \alpha}^{(\text {noisy) }}$ for the force projection coefficient in the presence of noise [we define, as before, $\Delta y_{\mu}\left(t_{i}\right)=y_{\mu}\left(t_{i+1}\right)-y_{\mu}\left(t_{i}\right)$ and $\left.\Delta x_{\mu}\left(t_{i}\right)=x_{\mu}\left(t_{i+1}\right)-x_{\mu}\left(t_{i}\right)\right]$ :

$$
\begin{aligned}
\hat{F}_{\mu \alpha}^{(\text {noisy })}= & \frac{1}{\tau} \sum_{i} \Delta y_{\mu}\left(t_{i}\right) c_{\alpha}\left(\mathbf{y}\left(t_{i}\right)\right) \\
= & \frac{1}{\tau} \sum_{i} \Delta x_{\mu}\left(t_{i}\right) c_{\alpha}\left(\mathbf{y}\left(t_{i}\right)\right) \\
& +\frac{1}{\tau} \sum_{i} \Delta t c_{\alpha}\left(\mathbf{y}\left(t_{i}\right)\right) \frac{\eta_{\mu}^{i+1}-\eta_{\mu}^{i}}{\Delta t} .
\end{aligned}
$$

There are two parts to the error due to measurement noise, one stemming from the noise in the position and the other from the noise in the velocity. We assume here that the former is relatively small, i.e., that we can write

$$
\begin{gathered}
c_{\alpha}[\mathbf{y}(t)] \approx \\
c_{\alpha}(\mathbf{x}(t))+\eta_{\mu}(t) \partial_{\mu} c_{\alpha}(\mathbf{x}(t)) \\
+\frac{\eta_{\mu} \eta_{\nu}}{2} \partial_{\mu \nu}^{2} c_{\alpha}(\mathbf{x}(t))+\ldots
\end{gathered}
$$

Then the average (over measurement noise) of the estimator for the force projection reads

$$
\left\langle\hat{F}_{\mu \alpha}^{\text {(noisy) }}\right\rangle=\hat{F}_{\mu \alpha}-\frac{\left\langle\eta_{\mu} \eta_{\nu}\right\rangle}{\Delta t} \int \partial_{\nu} c_{\alpha}(\mathbf{x}(t)) \frac{d t}{\tau}+\ldots
$$

This second term is a "dangerous" bias, as it diverges with $\Delta t \rightarrow 0$, which is symptomatic of the influence of measurement noise on force inference. Equation (F2) is thus impractical in this case.

In contrast, it is interesting to notice than when doing the same expansion with the velocity projection coefficients, we have

$$
\begin{aligned}
\hat{v}_{\mu \alpha}^{\text {(noisy })}= & \frac{1}{\tau} \sum_{i} \Delta y_{\mu}\left(t_{i}\right) c_{\alpha}\left(\frac{\mathbf{y}\left(t_{i}\right)+\mathbf{y}\left(t_{i+1}\right)}{2}\right) \\
= & \frac{1}{\tau} \sum_{i} \Delta x_{\mu}\left(t_{i}\right) c_{\alpha}\left(\frac{\mathbf{y}\left(t_{i}\right)+\mathbf{y}\left(t_{i+1}\right)}{2}\right) \\
& +\frac{1}{\tau} \sum_{i} \Delta t \frac{\eta_{\mu}^{i+1}-\eta_{\mu}^{i}}{\Delta t} c_{\alpha}\left(\frac{\mathbf{y}\left(t_{i}\right)+\mathbf{y}\left(t_{i+1}\right)}{2}\right)
\end{aligned}
$$

and

$$
\begin{aligned}
c_{\alpha}( & \left.\frac{\mathbf{y}\left(t_{i}\right)+\mathbf{y}\left(t_{i+1}\right)}{2}\right) \\
\approx & c_{\alpha}\left(\frac{\mathbf{x}\left(t_{i}\right)+\mathbf{x}\left(t_{i+1}\right)}{2}\right) \\
\quad & \quad \frac{\left(\eta_{\mu}^{i+1}+\eta_{\mu}^{i}\right)}{2} \partial_{\mu} c_{\alpha}\left(\frac{\mathbf{x}\left(t_{i}\right)+\mathbf{x}\left(t_{i+1}\right)}{2}\right)+\ldots
\end{aligned}
$$

Now all the dangerous terms in $1 / \Delta t$ have zero average. Indeed, averaging over the measurement noise,

$$
\begin{gathered}
\left\langle\frac{\left(\eta_{\mu}^{i+1}-\eta_{\mu}^{i}\right)}{\Delta t} \frac{\left(\eta_{\mu}^{i+1}+\eta_{\mu}^{i}\right)}{2} \partial_{\nu} c_{\alpha}\left(\frac{\mathbf{x}\left(t_{i}\right)+\mathbf{x}\left(t_{i+1}\right)}{2}\right)\right\rangle \\
=\frac{\left\langle\eta_{\mu}^{i+1} \eta_{\nu}^{i+1}-\eta_{\mu}^{i} \eta_{\nu}^{i}\right\rangle}{2 \Delta t} \partial_{\nu} c_{\alpha}\left(\frac{\mathbf{x}\left(t_{i}\right)+\mathbf{x}\left(t_{i+1}\right)}{2}\right)=0 .
\end{gathered}
$$

The reason for these useful cancellations is that by construction, the velocity projection coefficient is odd under time reversal of the trajectory; in contrast, all moments of the measurement noise are even under time reversal, as it is assumed to be time uncorrelated. Note that there remains a fluctuating term which is of the order $O(\sqrt{\Lambda / \tau \Delta t})$, where $\Lambda$ is the magnitude of the measurement noise variance. Up to this zero-mean error term, our estimator for the velocity projection coefficients is thus unaffected by measurement noise on time derivatives.

To obtain an unbiased estimator for the force, we may use the relation between Itô and Stratonovich integration for a variable $x$ which satisfies the stochastic differential equation [Eq. (A1)]:

$$
\begin{aligned}
& \frac{1}{\tau} \sum_{i} \Delta x_{\mu}\left(t_{i}\right) c_{\alpha}\left(\mathbf{x}\left(t_{i}\right)\right) \\
& =\frac{1}{\tau} \sum_{i} \Delta x_{\mu}\left(t_{i}\right) c_{\alpha}\left(\frac{x\left(t_{i+1}\right)+x\left(t_{i}\right)}{2}\right) \\
& \quad-D_{\mu \nu} \frac{1}{\tau} \sum_{i} \partial_{\nu} c_{\alpha}\left(\frac{x\left(t_{i}\right)+x\left(t_{i+1}\right)}{2}\right) \Delta t .
\end{aligned}
$$

We can therefore use for the force estimator

$\hat{F}_{\mu \alpha}=\hat{v}_{\mu \alpha}-D_{\mu \nu} \frac{1}{\tau} \sum_{i} \partial_{\nu} c_{\alpha}\left(\frac{y\left(t_{i}\right)+y\left(t_{i+1}\right)}{2}\right) \Delta t$,

where we see that $\hat{v}_{\mu \alpha}$ is unbiased by the noise, and the last term does not include a time derivative of the measurement and so is also under control.

Note that both the empirical information $\hat{I}_{b}$ and the estimated entropy production $\hat{\dot{S}}_{b}$ are now biased by the measurement noise, the bias being of order $O(1 /(\tau \Delta t))$. Thus, our treatment of the measurement noise remains incomplete, and if no other method is used to take care of the measurement noise, it requires sufficiently large $\tau$ as well as not too small time steps $\Delta t$. In addition, if the amplitude of the noise is not small compared to the typical spatial variation of the trajectory, then there are additional biases coming from evaluating the projectors at the wrong points.

Finally, in order to resolve the force correctly, the time step $\Delta t$ must not be too large: Indeed, force variations during the time step result in a blurring of the inferred force 
field. Specifically, the force variation over a time step is, on average, $\left\langle\Delta F_{\mu}\right\rangle \sim \Delta t F_{\nu} \partial_{\nu} F_{\mu}$. This results in a discretization bias $\delta \hat{F}_{\mu \alpha}$ in the force estimator [Eq. (F2)], the magnitude $\epsilon_{\text {discretization }}$ of which can be self-consistently estimated as

$\epsilon_{\text {discretization }}^{2}=\frac{\delta \hat{F}_{\nu \alpha} D_{\mu \nu}^{-1} \delta \hat{F}_{\mu \alpha}}{\hat{F}_{\nu \alpha} D_{\mu \nu}^{-1} \hat{F}_{\mu \alpha}} \sim \frac{\Delta t^{2}}{4 \hat{C}^{2}}\left\langle\left(\hat{F}_{\rho} \partial_{\rho} \hat{F}_{\mu}\right) D_{\mu \nu}^{-1}\left(\hat{F}_{\sigma} \partial_{\sigma} \hat{F}_{\mu}\right)\right\rangle$,

where $\hat{F}_{\mu}(\mathbf{x})=\hat{F}_{\mu \alpha} \hat{c}_{\alpha}(\mathbf{x})$ is the inferred force field, $\hat{C}=$ $\hat{F_{\nu \alpha}} D_{\mu \nu}^{-1} \hat{F_{\mu \alpha}} / 4$ is the inferred capacity, and $\langle\cdot\rangle$ denotes average over the trajectory. Note, however, that when using, as we suggest for "real" data, Eq. (F11) as an estimator for the force projections, the discretization error is only for the dissipative part of the force field, i.e., only on $\hat{v}$. Indeed, the second term in Eq. (F11) does not involve the time ordering of the data, and is therefore independent of $\Delta t$. Furthermore, the use of a Stratonovich average for the estimate of $\hat{v}_{\mu \alpha}$ reduces the squared error in Eq. (F12) by a factor of 4.
Comparing the discretization error estimate [Eq. (F12)] with the error stemming from the limited amount of information, Eq. (C24) allows us to self-consistently determine whether the limiting factor to force inference is the total trajectory length or the frame rate. This is particularly important for the optimization of the acquisition protocol in applications such as tracking of fluorescently labeled biological objects, where photobleaching limits the total number of frames that can be captured.

\section{APPENDIX G: INFERENCE IN THE PRESENCE OF AN INHOMOGENEOUS DIFFUSION COEFFICIENT}

We now provide proofs of the results presented in Sec. IV of the main text, regarding the inference of diffusion and drift in the presence of a state-dependent diffusion tensor. Our method of inference for the diffusion coefficient follows a similar logic to that of the inference of the force. We start with the local expression

$$
D_{\mu \nu}(\mathbf{x})=\frac{1}{2} \lim _{\Delta t \rightarrow 0}\left\langle\frac{[\mathbf{x}(t+\Delta t)-\mathbf{x}(t)]_{\mu}[\mathbf{x}(t+\Delta t)-\mathbf{x}(t)]_{\nu}}{\Delta t} \mid \mathbf{x}(t)=\mathbf{x}\right\rangle,
$$

and define the projections

$$
\begin{aligned}
D_{\mu \nu \alpha} & =\frac{1}{2} \int d \mathbf{x} P(\mathbf{x}) D_{\mu \nu}(\mathbf{x}) c_{\alpha}(\mathbf{x})=\int d \mathbf{x} \lim _{\Delta t \rightarrow 0}\left\langle\delta(\mathbf{x}(t)-\mathbf{x}) \frac{[\mathbf{x}(t+\Delta t)-\mathbf{x}(t)]_{\mu}[\mathbf{x}(t+\Delta t)-\mathbf{x}(t)]_{\nu}}{\Delta t}\right\rangle c_{\alpha}(\mathbf{x}) \\
& =\frac{1}{2}\left\langle\int d \mathbf{x} \delta(\mathbf{x}(t)-\mathbf{x}) \lim _{\Delta t \rightarrow 0} \frac{[\mathbf{x}(t+\Delta t)-\mathbf{x}(t)]_{\mu}[\mathbf{x}(t+\Delta t)-\mathbf{x}(t)]_{\nu}}{\Delta t} c_{\alpha}(\mathbf{x})\right\rangle \\
& =\frac{1}{2} \lim _{\Delta t \rightarrow 0}\left\langle\frac{[\mathbf{x}(t+\Delta t)-\mathbf{x}(t)]_{\mu}[\mathbf{x}(t+\Delta t)-\mathbf{x}(t)]_{\nu}}{\Delta t} c_{\alpha}(\mathbf{x})\right\rangle
\end{aligned}
$$

from which we get our estimator

$$
\hat{D}_{\mu \nu \alpha}=\frac{1}{\tau} \sum_{i=0}^{N} \Delta t \hat{d}_{\mu \nu}\left(t_{i}\right) \hat{c}_{\alpha}\left(\mathbf{x}\left(t_{i}\right)\right),
$$

where we define the local diffusion estimator,

$$
\hat{d}_{\mu \nu}\left(t_{i}\right)=\frac{\Delta x_{\mu}\left(t_{i}\right) \Delta x_{\nu}\left(t_{i}\right)}{2 \Delta t} .
$$

\section{Estimate of the error on the projected diffusion coefficient}

We now compute the typical error between the estimator $\hat{D}_{\mu \nu \alpha}$ and the exact projection coefficient $D_{\mu \nu \alpha}$. We work with the discrete version of the overdamped Langevin equation [Eq. (10)] written using the Itô convention:

$$
\begin{aligned}
\Delta x_{\mu}\left(t_{i}\right) & =x_{\mu}\left(t_{i+1}\right)-x_{\mu}\left(t_{i}\right) \\
& =\Phi_{\mu}\left(\mathbf{x}\left(t_{i}\right)\right) \Delta t+\sqrt{2 D\left(\mathbf{x}\left(t_{i}\right)\right)_{\mu \nu}} \Delta \xi_{\nu}^{t_{i}},
\end{aligned}
$$

where $\Delta \xi_{\nu}^{t_{i}}$ is a centered Gaussian variable with variance $\left\langle\Delta \xi_{\nu}^{t_{i}} \Delta \xi_{\mu}^{t_{i}}\right\rangle=\Delta t \delta_{\mu \nu} \delta_{i j}$. For error calculations, we consider only the leading-order terms in $\Delta t$ so that we can replace $\Delta x_{\mu}\left(t_{i}\right) \Delta x_{\nu}\left(t_{i}\right)$ by $2 D_{\mu \rho}^{1 / 2} D_{\nu \sigma}^{1 / 2} \Delta \xi_{\rho}^{t_{i}} \Delta \xi_{\sigma}^{t_{i}}$. Hence,

$$
\begin{aligned}
\hat{D}_{\mu \nu \alpha}-D_{\mu \nu \alpha}= & \frac{1}{N} \sum_{i=0}^{N} D_{\mu \rho}^{1 / 2}\left(\mathbf{x}\left(t_{i}\right)\right) D_{\nu \sigma}^{1 / 2}\left(\mathbf{x}\left(t_{i}\right)\right) \\
& \times\left(\frac{\Delta \xi_{\rho}^{t_{i}} \Delta \xi_{\sigma}^{t_{i}}}{\Delta t}-\delta_{\rho \sigma}\right) \hat{c}_{\alpha}\left(\mathbf{x}\left(t_{i}\right)\right) .
\end{aligned}
$$

We define the normalized (dimensionless) error 
$E_{\alpha}=\bar{D}_{\mu \nu}^{-1}\left(\hat{D}_{\mu \nu \alpha}-D_{\mu \nu \alpha}\right)=\frac{1}{N} \sum_{i=0}^{N} \tilde{D}_{\rho \sigma}\left(\mathbf{x}\left(t_{i}\right)\right) \zeta_{\rho \sigma}^{t_{i}} \hat{c}_{\alpha}\left(\mathbf{x}\left(t_{i}\right)\right)$

where $\bar{D}_{\mu \nu}$ is a reference constant diffusion matrix used for the normalization, which could be taken as the average diffusion tensor: $\bar{D}_{\mu \nu}=\int D_{\mu \nu}(\mathbf{x}) P(\mathbf{x}) d \mathbf{x}$. We also denote $\tilde{D}_{\rho \sigma}=D_{\rho \mu}^{1 / 2} \bar{D}_{\mu \nu}^{-1} D_{\nu \sigma}^{1 / 2}$ and $\zeta_{\rho \sigma}^{t_{i}}=\Delta \xi_{\rho}^{t_{i}} \Delta \xi_{\sigma}^{t_{i}} / \Delta t-\delta_{\rho \sigma}$. Note that $\left\langle\zeta_{\rho \sigma}^{t_{i}}\right\rangle=0$ and $\left\langle\zeta_{\rho \sigma}^{t_{i}} \zeta_{\mu \nu}^{t_{j}}\right\rangle=\delta_{i j}\left(\delta_{\rho \mu} \delta_{\sigma \nu}+\delta_{\rho \nu} \delta_{\sigma \mu}\right)$ :

$$
\begin{aligned}
\left\langle\zeta_{\rho \sigma}^{t_{i}} \zeta_{\mu \nu}^{t_{i}}\right\rangle & =\left\langle\left(\frac{\Delta \xi_{\rho}^{t_{i}} \Delta \xi_{\sigma}^{t_{i}}}{\Delta t}-\delta_{\rho \sigma}\right)\left(\frac{\Delta \xi_{\mu}^{t_{i}} \Delta \xi_{\nu}^{t_{i}}}{\Delta t}-\delta_{\mu \nu}\right)\right\rangle \\
& =\frac{\left\langle\Delta \xi_{\rho}^{t_{i}} \Delta \xi_{\sigma}^{t_{i}} \Delta \xi_{\mu}^{t_{i}} \Delta \xi_{\nu}^{t_{i}}\right\rangle}{\Delta t^{2}}-\delta_{\rho \sigma} \delta_{\mu \nu} \\
& =\delta_{\rho \mu} \delta_{\sigma \nu}+\delta_{\rho \nu} \delta_{\sigma \mu}
\end{aligned}
$$

using Wick's theorem in the last equality. The normalized squared error is then given by

$$
\left\langle E_{\alpha} E_{\alpha}\right\rangle=\frac{1}{N^{2}} \sum_{i=0}^{N} \sum_{j=0}^{N}\left\langle\zeta_{\mu \nu}^{t_{j}} \zeta_{\rho \sigma}^{t_{i}} \tilde{D}_{\rho \sigma}\left(\mathbf{x}\left(t_{i}\right)\right) \tilde{D}_{\mu \nu}\left(\mathbf{x}\left(t_{j}\right)\right) \hat{c}_{\alpha}\left(\mathbf{x}\left(t_{i}\right)\right) \hat{c}_{\alpha}\left(\mathbf{x}\left(t_{j}\right)\right)\right\rangle .
$$

We compute the leading order of this error, replacing $\hat{c}_{\alpha}\left(\mathbf{x}\left(t_{j}\right)\right)$ by $c_{\alpha}\left(\mathbf{x}\left(t_{j}\right)\right)$ :

$$
\begin{aligned}
& \frac{1}{N^{2}} \sum_{i=0}^{N} \sum_{j=0}^{N}\left\langle\zeta_{\mu \nu}^{t_{j}} \zeta_{\rho \sigma}^{t_{i}} \tilde{D}_{\rho \sigma}\left(\mathbf{x}\left(t_{i}\right)\right) \tilde{D}_{\mu \nu}\left(\mathbf{x}\left(t_{j}\right)\right) c_{\alpha}\left(\mathbf{x}\left(t_{i}\right)\right) c_{\alpha}\left(\mathbf{x}\left(t_{j}\right)\right)\right\rangle \\
& \quad=\frac{1}{N^{2}} \sum_{i=0}^{N}\left\langle\zeta_{\mu \nu}^{t_{i}} \zeta_{\rho \sigma}^{t_{i}}\right\rangle\left\langle\tilde{D}_{\rho \sigma}\left(\mathbf{x}\left(t_{i}\right)\right) \tilde{D}_{\mu \nu}\left(\mathbf{x}\left(t_{i}\right)\right) c_{\alpha}\left(\mathbf{x}\left(t_{i}\right)\right) c_{\alpha}\left(\mathbf{x}\left(t_{i}\right)\right)\right\rangle=\frac{1}{N^{2}} \sum_{i=0}^{N}\left\langle\tilde{D}_{\nu \mu}\left(\mathbf{x}\left(t_{i}\right)\right) \tilde{D}_{\mu \nu}\left(\mathbf{x}\left(t_{i}\right)\right) c_{\alpha}\left(\mathbf{x}\left(t_{i}\right)\right) c_{\alpha}\left(\mathbf{x}\left(t_{i}\right)\right)\right\rangle \\
& \quad=\frac{1}{N}\left\langle\int_{0}^{\tau} \frac{d t}{\tau} \tilde{D}_{\nu \mu}\left(\mathbf{x}\left(t_{i}\right)\right) \tilde{D}_{\mu \nu}\left(\mathbf{x}\left(t_{i}\right)\right) c_{\alpha}\left(\mathbf{x}\left(t_{i}\right)\right) c_{\alpha}\left(\mathbf{x}\left(t_{i}\right)\right)\right\rangle \\
& \quad \leq \frac{d\left(D_{\max }\right)^{2}}{N}\left\langle\int_{0}^{\tau} \frac{d t}{\tau} c_{\alpha}\left(\mathbf{x}\left(t_{i}\right)\right) c_{\alpha}\left(\mathbf{x}\left(t_{i}\right)\right)\right\rangle=\frac{\left(D_{\max }\right)^{2} N_{b}}{N}=\frac{\left(D_{\max }\right)^{2} N_{b} \Delta t}{\tau} .
\end{aligned}
$$

In the equality in the second line, we use that $\zeta_{\rho \sigma}^{t_{i}}$ is white in time correlated and centered, i.e., that it is uncorrelated with $\mathbf{x}\left(t_{j}\right)$ for $j \leq i$ and that $\left\langle\zeta_{\rho \sigma}^{t_{i}}\right\rangle=0$, which gives an Itô isometry type of result for the double sum. In the line before last, we pass to the continuous limit of the sum using $\tau=N \Delta t$. In the last line, we assume that $\tilde{D}_{\nu \mu}\left(\mathbf{x}\left(t_{i}\right)\right)$ is bounded from above in the domain. We denote by $D_{\max }$ the maximum eigenvalue of $\tilde{D}_{\nu \mu}\left(\mathbf{x}\left(t_{i}\right)\right)$ in the domain and bound $\tilde{D}_{\nu \mu}\left(\mathbf{x}\left(t_{i}\right)\right) \tilde{D}_{\mu \nu}\left(\mathbf{x}\left(t_{i}\right)\right) \leq d\left(\tilde{D}_{\max }\right)^{2}$.

Let us comment that the correction to the above result, due to the difference between $\hat{c}_{\alpha}\left(\mathbf{x}\left(t_{j}\right)\right)$ and $c_{\alpha}\left(\mathbf{x}\left(t_{j}\right)\right)$ can be bounded in a similar fashion as is done in Appendix C 3 , if one again uses the assumption that $\tilde{D}_{\nu \mu}\left(\mathbf{x}\left(t_{i}\right)\right)$ is bounded in the domain. This correction should result in a term of order $O\left(\tau^{-3 / 2}\right)$, which is subleading.

To summarize, we have the error estimate

$$
\left\langle\left\|\bar{D}_{\mu \nu}^{-1}\left(\hat{D}_{\mu \nu \alpha}-D_{\mu \nu \alpha}\right)\right\|^{2}\right\rangle \leq \frac{\left(D_{\max }\right)^{2} N_{b} \Delta t}{\tau}
$$

with $\tilde{D}_{\max }$ the maximum eigenvalue of $\tilde{D}_{\rho \sigma}=$ $D_{\rho \mu}^{1 / 2} \bar{D}_{\mu \nu}^{-1} D_{\nu \sigma}^{1 / 2}$ in the domain. Here the choice of normalization $\bar{D}$ is arbitrary, and it may be chosen as a diagonal matrix with the maximal diffusion coefficients in the domain on the diagonal, in a dimensionally consistent way (i.e., if there are directions in phase space with different units, each has its own maximal diffusion). In that case, $D_{\max }$ becomes of order unity.

\section{Inference of the diffusion coefficient with measurement noise}

As in Appendix F, we now consider the case where the exact trajectory is not known, but only a noisy approximation of it, due to imperfections of the measurement device. To correct for such a measurement noise, we suggest using the modified estimator

$$
\hat{D}_{\mu \nu \alpha}^{\text {(noisy) }}=\frac{1}{\tau} \sum_{i=0}^{N} \Delta t \hat{d}_{\mu \nu}^{\text {(noisy) }}\left(t_{i}\right) \hat{c}_{\alpha}\left(y\left(t_{i}\right)\right),
$$

where as in Eq. (13) of the main text,

$$
\begin{aligned}
\hat{d}_{\mu \nu}^{\text {(noisy })}\left(t_{i}\right)= & \frac{1}{4 \Delta t}\left[\Delta y_{\mu}\left(t_{i}\right) \Delta y_{\nu}\left(t_{i}\right)+\Delta y_{\mu}\left(t_{i-1}\right) \Delta y_{\nu}\left(t_{i-1}\right)\right. \\
& +2 \Delta y_{\mu}\left(t_{i-1}\right) \Delta y_{\nu}\left(t_{i}\right) \\
& \left.+2 \Delta y_{\mu}\left(t_{i}\right) \Delta y_{\nu}\left(t_{i-1}\right)\right]
\end{aligned}
$$


is the bias-corrected estimator proposed by Vestergaard et al. for homogeneous diffusion inference in the presence of measurement noise [50]. Indeed, the measurement noiseinduced terms compensate in Eq. (G13) thanks to the additional cross terms $\Delta y_{\mu}\left(t_{i-1}\right) \Delta y_{\nu}\left(t_{i}\right)$.

Let us compare the squared error for the corrected estimator [Eq. (G12)] with that for the estimator [Eq. (G3)]: On the one hand, the squared error for Eq. (G3) has a nonvanishing bias of order $\bar{D}^{-2} \Lambda^{2} / \Delta t^{2}$ due to measurement noise, while Eq. (G12) has only a contribution of order $\bar{D}^{-2} \Lambda^{2} /(\tau \Delta t)$, which vanishes for long trajectories. On the other hand, the squared error for the corrected estimator [Eq. (G12)] has an additional contribution coming from the signal due to the contributions to $\left\langle\zeta_{\rho \sigma}^{t_{i}} \zeta_{\mu \nu}^{t_{i}}\right\rangle$ from $\Delta y_{\mu}\left(t_{i-1}\right) \Delta y_{\nu}\left(t_{i}\right) / \Delta t+$ $\Delta y_{\mu}\left(t_{i}\right) \Delta y_{\nu}\left(t_{i-1}\right) / \Delta t$ when squared:

$$
\begin{aligned}
& \left\langle\frac{\Delta \xi_{\mu}^{t_{i-1}} \Delta \xi_{\rho}^{t_{i-1}}}{\Delta t}\right\rangle\left\langle\frac{\Delta \xi_{\nu}^{t_{i}} \Delta \xi_{\sigma}^{t_{i}}}{\Delta t}\right\rangle+\left\langle\frac{\Delta \xi_{\mu}^{t_{i}} \Delta \xi_{\rho}^{t_{i}}}{\Delta t}\right\rangle\left\langle\frac{\Delta \xi_{\nu}^{t_{i-1}} \Delta \xi_{\sigma}^{t_{i-1}}}{\Delta t}\right\rangle \\
& +\left\langle\frac{\Delta \xi_{\nu}^{t_{i-1}} \Delta \xi_{\rho}^{t_{i-1}}}{\Delta t}\right\rangle\left\langle\frac{\Delta \xi_{\mu}^{t_{i}} \Delta \xi_{\sigma}^{t_{i}}}{\Delta t}\right\rangle+\left\langle\frac{\Delta \xi_{\nu}^{t_{i}} \Delta \xi_{\rho}^{t_{i}}}{\Delta t}\right\rangle\left\langle\frac{\Delta \xi_{\mu}^{t_{i-1}} \Delta \xi_{\sigma}^{t_{i-1}}}{\Delta t}\right\rangle \\
& =2 \delta_{\mu \rho} \delta_{\nu \sigma}+2 \delta_{\nu \rho} \delta_{\mu \sigma}
\end{aligned}
$$

giving a squared error that is 4 times larger than that of the biased estimator in Eq. (G3). Therefore, there is a trade-off where for short trajectories with sufficiently small measurement noise, the estimator (G3) may outperform the corrected estimator, but the (squared) error on it would saturate at $\bar{D}^{-2} \Lambda^{2} / \Delta t^{2}$ for sufficiently long trajectories, for which the error on the corrected estimator would continue decreasing. This behavior is demonstrated in Fig. 8(d) in the main text.

\section{Drift inference for an inhomogeneous diffusion coefficient}

We now turn to the inference of the Itô drift [Eq. (11)]. As we discuss in the main text, in the presence of inhomogeneous diffusion the force estimator we use before [Eq. (C8)] becomes an estimator for the drift:

$$
\begin{aligned}
\hat{\Phi}_{\mu \alpha} & =\frac{1}{\tau} \int^{\mathrm{It} \hat{o}} \hat{c}_{\alpha}(\mathbf{x}) d \mathbf{x}_{t}^{\mu} \\
& =\underbrace{\frac{1}{\tau} \int_{0}^{\tau} \hat{c}_{\alpha}(\mathbf{x}) \Phi_{\mu}(\mathbf{x}) d t}_{\Phi_{\mu \alpha}^{\tau}}+\underbrace{\frac{1}{\tau} \int^{\mathrm{It} \hat{o}} \hat{c}_{\alpha}(\mathbf{x}) \sqrt{2} D_{\mu \nu}^{1 / 2}(\mathbf{x}) d \xi_{t}^{\nu}}_{Z_{\mu \alpha}},
\end{aligned}
$$

$(\mathrm{G} 15)$

where as in Appendix C, we define $\Phi_{\mu \alpha}^{\tau}$ as the projection of the exact drift onto $\hat{c}_{\alpha}(\mathbf{x})$. This estimator is, however, biased by measurement noise, as we discuss in Appendix F. To circumvent this limitation and make our estimators applicable to real, noisy data, we use again the relation between the Itô and Stratonovich integrals. As in Appendix $\mathrm{F}$, we thus relate $\hat{\Phi}_{\mu \alpha}$ to $\hat{v}_{\mu \alpha}$, which can be inferred as before (it is unaffected by inhomogeneous diffusion) and is unbiased by measurement noise. We have

$$
\begin{aligned}
\frac{1}{\tau} \int^{\mathrm{Itô}} \hat{c}_{\alpha}(\mathbf{x}) d \mathbf{x}_{t}^{\mu} & =\frac{1}{\tau} \int^{\mathrm{Strat}} \hat{c}_{\alpha}(\mathbf{x}) d \mathbf{x}_{t}^{\mu}-\frac{1}{\tau} \int D_{\mu \nu}(\mathbf{x}) \partial_{\nu} \hat{c}_{\alpha}(\mathbf{x}) d t \\
& =\hat{v}_{\mu \alpha}-\frac{1}{\tau} \int D_{\mu \nu}(\mathbf{x}) \partial_{\nu} \hat{c}_{\alpha}(\mathbf{x}) d t .
\end{aligned}
$$

To make this a practical estimator, however, one needs to substitute the unknown $D_{\mu \nu}(\mathbf{x})$ with an accessible value. Using the standard diffusion estimator [Eq. (G4)] results in an expression that is mathematically equivalent to Eq. (G15): It is correct with ideal data but flawed in the presence of measurement noise. With ideal data, we thus recommend the use of Eq. (G15), which is significantly less complex computationally. In the presence of measurement noise, using the modified diffusion estimator $\hat{d}_{\mu \nu}^{\text {(noisy) }}(t)$ [Eq. (G13)] corrects for the bias induced by measurement noise. This yields our drift projection estimator adapted to systems with measurement noise, Eq. (14) of the main text:

$$
\hat{\Phi}_{\mu \alpha}=\hat{v}_{\mu \alpha}-\frac{1}{\tau} \sum_{i} \hat{d}_{\mu \nu}^{(\text {noisy })}\left(t_{i}\right) \partial_{\nu} \hat{c}_{\alpha}\left(\mathbf{x}\left(t_{i}\right)\right) \Delta t
$$

Indeed, $\left\langle\hat{d}_{\mu \nu}^{\text {(noisy) }}(t) \partial_{\nu} \hat{c}_{\alpha}(\mathbf{x}(t))\right\rangle=\left\langle D_{\mu \nu}(\mathbf{x}(t)) \partial_{\nu} \hat{c}_{\alpha}(\mathbf{x}(t))\right\rangle$ : To first order, the use of the modified local diffusion estimator does not result in a bias in Eq. (G17).

\section{Estimate of the error on the projected drift}

Here we estimate the error on the inference of $\hat{\Phi}_{\mu \alpha}$. To this end, we employ the Itô version of the estimator, Eq. (G15). The error on Eq. (G17) has a similar form but is analytically less tractable.

We thus want to estimate the relative magnitude of the error term $Z_{\mu \alpha}$ in Eq. (G15). The statistics of $Z_{\mu \alpha}$ can be derived following the derivation in Appendix C 3 , except that now the diffusion coefficient depends on $\mathbf{x}$. Thus, the normalized error $W_{\mu \alpha}$ is defined using the average diffusion coefficient $\bar{D}_{\mu \nu}$, and the calculations go through resulting in the same asymptotic behavior. However, now the variance of the error reads

$$
\left\langle Z_{\mu \alpha} Z_{\nu \beta}\right\rangle=\frac{2}{\tau}\left\langle D_{\mu \nu} c_{\alpha} c_{\beta}\right\rangle[1+O(1 / \sqrt{\tau})],
$$

where the space dependence of $D_{\mu \nu}$ prevents us from using the orthonormality of $c_{\alpha}$. We thus have

$$
\begin{aligned}
& \left\langle\left(\hat{\Phi}_{\mu \alpha}-\Phi_{\mu \alpha}^{\tau}\right)\left(\hat{\Phi}_{\nu \beta}-\Phi_{\nu \beta}^{\tau}\right)\right\rangle \\
& \quad=\frac{2}{\tau}\left\langle D_{\mu \nu} c_{\alpha} c_{\beta}\right\rangle[1+O(1 / \sqrt{\tau})] .
\end{aligned}
$$


Finally, we can normalize by the average diffusion tensor $\bar{D}_{\mu \nu}$ to obtain the estimate

$$
\left\langle\left(\hat{\Phi}_{\mu \alpha}-\Phi_{\mu \alpha}^{\tau}\right) \bar{D}_{\mu \nu}^{-1}\left(\hat{\Phi}_{\nu \beta}-\Phi_{\nu \beta}^{\tau}\right)\right\rangle \leq \frac{2 n_{b}}{\tau} D_{\max },
$$

where we define $D_{\max }$ as the maximal eigenvalue of the matrix $\bar{D}_{\mu \rho}^{-1} D_{\rho \nu}(\mathbf{x})$ in the domain.

Finally, we note that in our method, the inferred physical force $\hat{F}_{\mu}(\mathbf{x})$ is obtained in Eq. (15) by combining the drift with the divergence of the inferred diffusion tensor. As there is no control of the error on this latter term-the error on the gradient is a priori independent of the error on the function estimate, in the absence of regularity assumptions-we cannot provide an error estimate for the inferred physical force.

\section{APPENDIX H: MODEL DETAILS AND SIMULATION PARAMETERS FOR NUMERICAL RESULTS}

\section{Overdamped Langevin simulations}

To benchmark our stochastic force inference method, we test it on several simple models of Brownian dynamics. We discretize the overdamped Langevin equation $\dot{x}_{\mu}=$ $F_{\mu}+\xi_{\mu}$ into

$$
\mathbf{x}(t+d t)=\mathbf{x}(t)+d t \mathbf{F}(\mathbf{x}(t))+\sqrt{2 \mathbf{D} d t} \zeta,
$$

or, in the case of a state-dependent diffusion tensor inducing multiplicative noise,

$$
\begin{aligned}
\mathbf{x}(t+d t)= & \mathbf{x}(t)+d t \mathbf{F}(\mathbf{x}(t))+\sqrt{2 \mathbf{D}(\mathbf{x}(t)) d t} \zeta \\
& +d t \nabla \cdot \mathbf{D}(\mathbf{x}(t)) .
\end{aligned}
$$

Here, $\zeta$ is a vector of independent normal random variables with zero mean and unit variance. Again, the force here includes the mobility matrix: The system is out of equilibrium if $\mathbf{D}^{-1} \mathbf{F}(\mathbf{x})$ does not derive from a potential, regardless of whether this comes from violations of fluctuation-dissipation relations (such as interacting components at different temperatures), nonreciprocal interactions, or the presence of curl in the external force fields. Note that in order to ensure numerical stability of this equation, the interval $d t$ must be sufficiently small, while SFI can accommodate a moderately large value of $d t$ (see Appendix A). We therefore run the simulations at a higher rate than the input for SFI; the value of $\Delta t$ indicated in the parameters is that of the SFI input, while the elementary time step used to generate the trajectories is denoted $d t$. All simulations presented here have an initial state preequlibrated.

In the simulations presented in this article, the diffusion matrix is assumed to be known, except in Fig. 8 where inferring it is part of the object of the simulations. In all other figures, it could, however, be inferred using our method (but fitting it only with a constant). In general, in the strong-noise cases considered in this article, inferring the diffusion coefficient is significantly less demanding than force inference and results in very little additional error.

\section{2D Ornstein-Uhlenbeck processes (Fig. 3)}

The first model we benchmark our method on is a $2 \mathrm{D}$ process in a linear trap, also known as an OrnsteinUhlenbeck process. We consider here an anisotropic equilibrium process with isotropic diffusion; we set the diffusion to unity, $D_{\mu \nu}=\delta_{\mu \nu}$. The force field is $F_{\mu}=$ $-\Omega_{\mu \nu}\left(x_{\nu}-x_{\mu}^{0}\right)$ [black arrows in Fig. 3(a)], where we choose

$$
\mathbf{x}^{0}=\left(\begin{array}{l}
0 \\
0
\end{array}\right), \quad \Omega=\left(\begin{array}{cc}
1 & 0.5 \\
0.5 & 1
\end{array}\right) .
$$

We use a simulation time step $d t=0.005$ and $\Delta t=0.01$. The trajectory presented and analyzed in Fig. 3(a) of the main text has a length $N_{\text {samples }}=4000$. It is analyzed by SFI with basis $b=\left\{1, x_{1}, x_{2}\right\}$. The inferred projected force field on this basis [blue arrow in Fig. 3(a)] has the form $\hat{F}_{\mu}(\mathbf{x})=-\hat{\Omega}_{\mu \nu}\left(x_{\nu}-\hat{x}_{0}\right)$, where the $N_{b}=6$ inferred parameters are

$$
\hat{x}_{0}=\left(\begin{array}{l}
0.27 \\
0.13
\end{array}\right), \quad \hat{\Omega}=\left(\begin{array}{ll}
1.15 & 0.27 \\
0.42 & 0.76
\end{array}\right) .
$$

Quantitatively, as we mention in the main text, this results in a (squared) relative error on the inferred projection coefficient $\left[\left(\hat{F}_{\mu \alpha}-F_{\mu \alpha}\right) D_{\mu \nu}^{-1}\left(\hat{F}_{\nu \alpha}-F_{\nu \alpha}\right)\right] /\left[\hat{F}_{\mu \alpha} D_{\mu \nu}^{-1} \hat{F}_{\nu \alpha}\right]=0.15$. The inferred information along this trajectory is $\hat{I}_{b}=$ $\hat{F}_{\mu \alpha} D_{\mu \nu}^{-1} \hat{F}_{\nu \alpha}=19.1$ [i.e., 27.6 bits with the $1 / \log (2)$ natto-bit conversion factor]. The self-consistent confidence interval for this error is $N_{b} / 2 \hat{I}_{b}=0.16$ : The actual error is thus within the confidence interval.

It is interesting to note that the inferred matrix $\hat{\Omega}$ [Eq. (H4)] is not symmetric, meaning that the inferred model is out of equilibrium (it exhibits phase-space cycling). This does not, however, result in significant entropy production. Indeed, the inferred entropy produced is $\hat{\Delta S}=0.5 k_{B}$.

In Fig. 3(b) of the main text, we study the statistics of the relative error obtained over 64 realizations of trajectories of the same model, with varying length $N_{\text {samples }}=$ $2^{4}, 2^{5}, \ldots, 2^{17}, 2^{18}$. We present the average (and standard deviation, blue symbols and error bars) of the squared relative error $\left[\left(\hat{F}_{\mu \alpha}-F_{\mu \alpha}\right) D_{\mu \nu}^{-1}\left(\hat{F}_{\nu \alpha}-F_{\nu \alpha}\right)\right] /\left[\hat{F}_{\mu \alpha} D_{\mu \nu}^{-1} \hat{F}_{\nu \alpha}\right]$; the average self-consistent estimate of this error $N_{b} / 2 \hat{I}_{b}$ (orange solid curve) and the asymptotic convergence to $N_{b} / 2 \tau C_{b}$, i.e., the actual information per degree of freedom (black dashed line). These quantities match quantitatively 
in the long trajectory limit, as predicted from our analytical reasoning (Appendix C). Interestingly, in the regime where there is little information available in the trajectory, our self-consistent formula reliably predicts a relative error of order 1, consistent with the fact that there is no signal.

\section{6D circulating Ornstein-Uhlenbeck processes (Fig. 4)}

The next example we use to test SFI is another OrnsteinUhlenbeck process with force $F_{\mu}=-\Omega_{\mu \nu}\left(x_{\nu}-x_{\mu}^{0}\right)$, but this time with several complications: It is high dimensional $(d=6)$, with anisotropic diffusion and trapping, and such that we exert a torque in a given plane. We challenge our method by applying it to the short trajectories displayed in Fig. 4(a) in the main text, and even further in Fig. 4(b) in the presence of strong measurement noise.

The diffusion and harmonic trapping matrices are obtained as random matrices constructed to have a moderate degree of anisotropy. The diffusion matrix is symmetric, while the confinement is not and induces circulation. Specifically, we choose

$$
\begin{aligned}
\boldsymbol{\Omega} & =\left(\begin{array}{cccccc}
1.34 & -0.25 & -0 . & 0.73 & 0.38 & 0.23 \\
-0.07 & 1.77 & -0.45 & 1.92 & 0.88 & -0.09 \\
0.24 & 0.52 & 0.81 & -0.63 & 0.05 & 0.97 \\
-0.24 & -1.14 & 0.52 & 0.93 & -0.32 & -0.69 \\
0.16 & -0.01 & 0.07 & 0.66 & 0.92 & -0.02 \\
0.51 & 0.52 & 0.27 & 0.79 & 0.61 & 2.45
\end{array}\right), \\
\mathbf{D} & =\left(\begin{array}{cccccc}
1.92 & 1.27 & 0.29 & -0.18 & 0.2 & -0.02 \\
1.27 & 1.87 & 0.26 & -0.1 & 0.11 & -0.25 \\
0.29 & 0.26 & 0.98 & -0.45 & 0.06 & 0.09 \\
-0.18 & -0.1 & -0.45 & 1.03 & -0.17 & -0.15 \\
0.2 & 0.11 & 0.06 & -0.17 & 0.84 & 0.09 \\
-0.02 & -0.25 & 0.09 & -0.15 & 0.09 & 0.81
\end{array}\right),
\end{aligned}
$$

and $\mathbf{x}_{0}=0$. Our simulation parameters are $\Delta t=0.05$ and $d t=0.01$. The trajectory presented in Fig. 4(a) has $N_{\text {samples }}=400$ points, and the three plots correspond to three projections of the same trajectory, respectively (from left to right), along directions $\left(x_{1}, x_{2}\right),\left(x_{3}, x_{4}\right)$, and $\left(x_{5}, x_{6}\right)$.

In Fig. 4(c), we present the results of SFI at linear order $\left(b=\left\{1, x_{\mu}\right\}\right)$ for the specific trajectory displayed in Fig. 4(a). The inferred parameters are

$$
\begin{aligned}
\hat{x}_{0}=\left(\begin{array}{c}
-0.86 \\
-0.64 \\
-0.29 \\
-0.46 \\
-0.25 \\
0.25
\end{array}\right), \\
\hat{\Omega}=\left(\begin{array}{ccccccc}
2.38 & -1.24 & 0.47 & 0.4 & 0.19 & 0.29 \\
0.96 & 1.06 & -1.01 & 0.92 & 1.59 & -0.91 \\
-0.16 & 0.44 & 1.09 & -1.13 & 0.58 & 0.96 \\
0.18 & -1.36 & 1.07 & 1.27 & -0.91 & -0.87 \\
0.61 & -0.28 & -0 . & 0.36 & 1.01 & 0.22 \\
0.25 & 0.29 & 0.86 & 0.91 & 0.29 & 3 .
\end{array}\right),
\end{aligned}
$$

with a squared relative error of 0.24 , consistent with the self-consistent estimate $N_{b} / 2 \hat{I}_{b}=0.22$.

We show in Fig. 4(c) in the main text a 2D slice of the inferred force field (blue) and the exact force field (black). This slice is chosen as the plane of maximal inferred circulation. To determine this plane, we consider the nondimensionalized velocity projection coefficients $R_{\alpha \beta}=$ $C_{\alpha \mu}^{-1 / 2} \hat{v}_{\mu \beta}$ with $\mathbf{C}$ the covariance matrix of the data. With this choice of normalization, the rows and columns of $\mathbf{R}$ are normalized in the same way, and it thus makes sense to consider its antisymmetric part to quantify circulation. The eigenvalues of $\frac{1}{2}\left(R_{\alpha \beta}-R_{\beta \alpha}\right)$ are imaginary and come in conjugate pairs. We define the inferred principal circulation plane as the real-space plane (u,v) spanned by $\left(C_{\mu \alpha}^{1 / 2} r_{\alpha}^{1}, C_{\mu \alpha}^{1 / 2} r_{\alpha}^{2}\right)$, where $\left(r_{\alpha}^{1}, r_{\alpha}^{2}\right)$ is the pair of eigenvectors of $\mathbf{R}$ associated with the eigenvalue of largest norm. We compare this inferred plane to the exact plane of maximal circulation $\left(\mathbf{u}^{0}, \mathbf{v}^{0}\right)$ obtained through the same procedure but with an asymptotically long trajectory $\left(N_{\text {steps }}=2.10^{6}\right)$. In Fig. 4(e), we present the statistics of the angular error in this cycle detection. This angular error is defined as $\delta=\left\|\mathbf{u}-\left(\mathbf{u}^{0} \cdot \mathbf{u}\right) \mathbf{u}^{0}-\left(\mathbf{v}^{0} \cdot \mathbf{u}\right) \mathbf{v}^{0}\right\|^{2}+\left\|\mathbf{v}-\left(\mathbf{u}^{0} \cdot \mathbf{v}\right) \mathbf{u}^{0}-\left(\mathbf{v}^{0} \cdot \mathbf{v}\right) \mathbf{v}^{0}\right\|^{2}$, where $(\mathbf{u}, \mathbf{v})$ and $\left(\mathbf{u}^{0}, \mathbf{v}^{0}\right)$ are the pairs of orthogonal unit vectors defining the inferred and exact maximal circulation planes, respectively. This error is equal to 0.12 for the trajectory presented in Fig. 4(a), and decays to zero as $\delta \sim$ $\tau^{-1}$ with increasing trajectory length, as the inferred matrix $\hat{\Omega}$ converges to $\Omega$. Figure 4(f) shows the statistics of the debiased entropy production, $\hat{\dot{S}}-2 N_{b} / \tau$.

\section{a. Measurement noise}

In Fig. 4(b), we present the same trajectories as in Fig. 4(a), with an added challenge to force detection: a strong "measurement noise," i.e., a time-uncorrelated error 
on the input data $x_{\mu}$. We model such a noise by adding Gaussian white noise to each coordinate of $x_{\mu}$, with standard deviation equal to 0.5 (half the standard deviation of the data). In the presence of such time-uncorrelated noise, the estimate of $\dot{x}$ becomes strongly noisy, and we have to use the modified estimator for $\hat{F}_{\mu \alpha}$, Eq. (F11). With this estimator, we infer

$$
\begin{aligned}
\hat{x}_{0}=\left(\begin{array}{c}
-0.83 \\
-0.64 \\
-0.07 \\
-0.51 \\
-0.24 \\
0.12
\end{array}\right), \\
\hat{\Omega}=\left(\begin{array}{ccccccc}
1.92 & -1.04 & 0.26 & -0.09 & 0.18 & -0.26 \\
1.07 & 0.71 & -1.12 & 0.28 & 1.01 & -1.31 \\
-0.19 & 0.48 & 0.88 & -1.19 & 0.21 & 0.83 \\
0.03 & -0.66 & 0.78 & 0.92 & -0.2 & -1.12 \\
0.27 & 0.06 & -0.04 & -0.1 & 1.01 & -0.17 \\
0.21 & 0.23 & 0.45 & 0.67 & 0.32 & 1.91
\end{array}\right),
\end{aligned}
$$

with a squared relative error of 0.6 on $\hat{F}_{\mu \alpha}$ and an angular error on cycle detection of 0.156 .

\section{Nonlinear obstacle process}

[Figs. 5(a), 5(c), 5(e), 5(g), 5(i)]

In Fig. 5(a), we study the case of a 2D stochastic process with circulation in a nonlinear force field using stochastic force inference with a polynomial basis at different orders. The force field we use is

$$
\begin{aligned}
& F_{\mu}(\mathbf{x})=-\Omega_{\mu \nu} x_{\nu}+\alpha e^{-x^{2} / 2 \sigma^{2}} x_{\mu} \\
& \text { with } \quad \alpha=10, \quad \Omega=\left(\begin{array}{cc}
2 & 2 \\
-2 & 2
\end{array}\right),
\end{aligned}
$$

which is a nonpolynomial force field; i.e., it cannot be captured exactly in our choice of basis. We use isotropic diffusion with $D=1$. We simulate this process with $\Delta t=$ 0.01 and $d t=0.001$; the trajectory in Fig. 5(a) has $N_{\text {samples }}=4096$. We perform SFI on the trajectory with a polynomial basis at orders $n=1,3,5$ in Figs. 5(c), 5(e), and $5(\mathrm{~g})$; note that as the force field is odd under reversal $\mathbf{x} \rightarrow-\mathbf{x}$, the even orders in the polynomial expansion do not contribute to it [as apparent in the $n$ dependence of the capacity in Fig. 5(i)]. The bootstrapped trajectories presented on the right column of Figs. 5(c), 5(e), and 5(g) are obtained using the inferred projected force field $\hat{F}_{\mu \alpha} \hat{c}_{\alpha}(\mathbf{x})$ to simulate new trajectories with the same starting point, $\tau$, $d t$ and $\Delta t$ as the original trajectory.

In Fig. 5(i), we present the capacity $C_{b}$ and entropy production $\dot{S}_{b}$ captured by the projection of a long trajectory with $N_{\text {samples }}=2^{18}$ onto three different bases:

(i) Polynomials of order $n=0, \ldots, 7$

(ii) Fourier modes of order $n=0, \ldots, 7$; specifically, we use all functions of the form $\cos \left(2 \pi \sum_{\mu} k_{\mu}\left(x_{\mu}-\right.\right.$ $\left.\left.\left\langle x_{\mu}\right\rangle\right) / R_{\mu}\right)$ and $\sin \left(2 \pi \sum_{\mu} k_{\mu}\left(x_{\mu}-\left\langle x_{\mu}\right\rangle\right) / R_{\mu}\right)$ with non-negative integers $k_{\mu}$ such that $\sum_{\mu} k_{\mu} \leq n$. Here we choose $R_{\mu}$ to be 1.05 times the diameter of the trajectory in direction $\mu$.

(iii) A constant-by-part grid coarse graining with $n=$ $2 \ldots, 7$ grid cells in each direction centered on $\left\langle x_{\mu}\right\rangle$ and with width $R_{\mu}$.

\section{Lorenz process [Figs. 5(b), 5(d), 5(f), 5(h), 5(j)]}

Our second nonlinear process is a stochastic variant of a popular model for dynamical systems, the Lorenz system [46]. Its 3D Brownian dynamics is described by the force field

$F_{x}=s(y-x), \quad F_{y}=r x-y-z x, \quad F_{z}=x y-b z$.

In our simulations, we employ the parameters $r=10, s=3$, and $b=1$. Diffusion is isotropic with $D=1$. We use $\Delta t=d t=0.02$, and the trajectory in Fig. 5(b) has $N_{\text {samples }}=2^{12}$. All images of trajectories are in the $(x z)$ plane. It should be noted that this force field is polynomial of order two, implying that it can be fully captured by the order $n=2$ of our polynomial expansion. Indeed, with polynomial SFI at orders two and three [Figs. 5(f) and 5(h)], we capture precisely the force field, and bootstrapped trajectories are very similar to the original data. As apparent in Fig. 5(j), the order $n=1$ polynomial approximation captures only a fraction of the capacity and entropy production. Interestingly, the order $n=2$ polynomial approximation captures the whole capacity, but not the full entropy production, as there are nonzero exchange terms with higher-order moments (corresponding to the fact that the logarithm of the PDF is not itself a polynomial).

\section{Active Brownian particles simulations (Fig. 7)}

The next system that we study in this article corresponds to a model of self-propelled Brownian particles mimicking in a somewhat realistic manner experimental systems such as studied in Ref. [58]. Specifically, we simulate $N_{\text {particles }}=25$ self-propelled 2D particles, each characterized by its coordinates $\mathbf{x}$ and orientation $\theta$. These particles interact through soft repulsive pair interactions $f(r)$ between particles at distance $r$, are self-propelled toward the direction $\theta$ at velocity $v$, and are harmonically confined with strength $\omega$ : The force exerted on particle $i$ is thus, 


$$
\mathbf{F}_{i}=-\omega \mathbf{x}_{i}+v\left(\begin{array}{c}
\cos \theta_{i} \\
\sin \theta_{i}
\end{array}\right)-\sum_{j \neq i} f\left(r_{i j}\right) \frac{\mathbf{r}_{i j}}{r_{i j}},
$$

where $\mathbf{r}_{i j}=\mathbf{x}_{j}-\mathbf{x}_{j}$. The angle $\theta$ is freely diffusing (note that we could include alignment interactions in this model). In our simulations, we use $f(r)=1 /\left(r^{2}+1\right), \omega=0.2$, $v=1$, isotropic diffusion with $D=1$ in spatial coordinates, and angular diffusion with $D_{\theta}=0.1$. We use a large sampling time step $\Delta t=1$, while the simulation step is $d t=0.01$. The number of frames for our study is very limited $N_{\text {frames }}=25$, with significant positional and angular measurement noise (on both $x, y$, and $\theta$ with standard deviation 0.4). These limitations are chosen to mimic those of experimental data. Note that we assume that the identity of the particles can be tracked along the trajectory.

\section{a. Symmetrization of the forces}

Each of the 25 particles being characterized by 3 degrees of freedom, the phase space of this system is 75 dimensional, making any "brute-force" approximation of the force field in phase space hopeless: Even a simple form of such a linear polynomial [which would be a terrible approximation of Eq. (H10)] would have 5700 variables. Here we propose to use a more subtle projection basis, making use of the invariance of the force field when exchanging two particles. More precisely, instead of using a projection basis $b_{\alpha}\left(\left\{\mathbf{x}_{i}\right\}_{i=1, \ldots, N_{\text {particles }}}\right)$ that depends on each phase-space coordinate in an explicit way, we project on symmetrized functions $b_{\alpha}\left(\mathbf{x}_{i},\left\{\mathbf{x}_{j}\right\}_{j \neq i}\right)$ that consider the interaction between one particle $i$ and all others, regardless of the identity of $i$. The projected force field thus consists of an approximation of the force on any particle $i$ as

$$
F_{i, \mu} \approx F_{\mu \alpha} c_{\alpha}\left(\mathbf{x}_{i},\left\{\mathbf{x}_{j}\right\}_{j \neq i}\right),
$$

where, crucially, the projection coefficient $F_{\mu \alpha}$ and the projector $c_{\alpha}$ are independent of the identity of $i$. This drastically reduces the number of degrees of freedom of our approximation: Now the data on each particle contribute to the inference of the same coefficients $F_{\mu \alpha}$, and thus, a large number of particles actually facilitates force inference. These additional symmetry constraints on the projection do not fit, strictly speaking, in the framework developed in the rest of this article. Specifically, the orthonormalization of the projector is now performed with an additional average over all particles:

$$
\begin{aligned}
\hat{c}_{\alpha}= & \hat{B}_{\alpha \beta} b_{\beta} \text { with } \\
\hat{B}_{\alpha \beta}= & \frac{1}{\tau N_{\text {particles }}} \sum_{i} \int d t b_{\alpha}\left(\mathbf{x}_{i}(t),\left\{\mathbf{x}_{j}(t)\right\}_{j \neq i}\right) \\
& \times b_{\beta}\left(\mathbf{x}_{i}(t),\left\{\mathbf{x}_{j}(t)\right\}_{j \neq i}\right),
\end{aligned}
$$

and all integrals are adapted accordingly. For instance, the Itô integral for the force projection now reads

$$
\begin{aligned}
\hat{F}_{\mu \alpha}= & \frac{1}{\tau N_{\text {particles }}} \sum_{i} \sum_{t}\left(x_{i, \mu}(t+\Delta t)-x_{i, \mu}(t)\right) \\
& \times \hat{c}_{\alpha}\left(\mathbf{x}_{i}(t),\left\{\mathbf{x}_{j}(t)\right\}_{j \neq i}\right)
\end{aligned}
$$

with $\Delta t$ the time step.

\section{b. Choice of the basis}

So far, we use only the indiscernibility of the particles, without any assumption on the nature of their interactions: Eq. (H11) is completely generic and could, in principle, approximate any type of interaction-provided that the choice of projection basis is adapted. For instance, a natural choice would be to expand the interaction in single-particle terms (i.e., external fields), pair interactions, and possibly higher orders as

$$
\begin{aligned}
F_{i, \mu} \approx & F_{\mu \alpha}^{(1)} c_{\alpha}^{(1)}\left(\mathbf{x}_{i}\right)+F_{\mu \beta}^{(2)} \sum_{j \neq i} c_{\beta}^{(2)}\left(\mathbf{x}_{i}, \mathbf{x}_{j}\right) \\
& +F_{\mu \gamma}^{(3)} \sum_{j, k \neq i} c_{\gamma}^{(3)}\left(\mathbf{x}_{i}, \mathbf{x}_{j}, \mathbf{x}_{k}\right)+\cdots
\end{aligned}
$$

where $c^{(1)}, c^{(2)}, c^{(3)} \ldots$ are the respective projectors onto the space spanned by the one-, two- and three-body interaction terms in the basis. It is important to note that these projectors should be orthonormalized as a whole, either hierarchically (through the Gram-Schmidt process, for instance, by orthonormalizing the one-body term, then the two-body term with respect to itself, and the one-body term, etc.) or in a single step as in Eq. (H12) but with the index $\alpha$ now understood as comprising all terms in the expansion.

Let us also note that while polynomials constitute a natural "default" basis for generic processes in an unstructured phase space, no such natural choice exists for the interaction terms. Symmetries can serve as a guide: For instance, for radially or spherically symmetric particles, the magnitude of the pair interaction should depend on the distance $r_{i j}$ between particles. The use of such symmetries warrants some caution: Indeed, the choice of projection basis should be compatible with these symmetries. For instance, for radial symmetry, the basis $b=$ $\left\{\left(\mathbf{x}_{i}, \mathbf{x}_{j}\right) \mapsto r_{i j}^{n}\right\}_{n=0,1,2, \ldots}$, i.e., polynomials in the distance between particles, is not adapted. Indeed, a force written as a linear combination of these functions would transform as a scalar under rotations, not as a vector. Instead, $b=$ $\left\{\left(\mathbf{x}_{i}, \mathbf{x}_{j}\right) \mapsto r_{i j, \mu} r_{i j}^{n-1}\right\}_{\mu=1, \ldots, d, n=0,1,2, \ldots}$ would be adapted. This does not constrain the force to be invariant under rotation, but allows it. Finally, let us note that while this choice is fine, it is not great: Indeed, polynomials in $r$ put most of their weight in the far field, i.e., in interaction between faraway particles. SFI will thus put the most 
weight on capturing the tail of the interaction. In most cases, interactions decay with distance, and it is more interesting to capture the details of the interaction forces between nearby particles. For this reason, decaying functions of $r$, such as inverse power laws or exponentials, are better adapted. We finally note that non-power-law functions typically have a characteristic scale, or shape parameters. These parameters are not optimized upon by SFI, which fits only the signal as a linear combination of the basis functions: The outcome will thus depend on the choice of parameter. While such shape parameters could, in principle, be optimized upon (for instance, to maximize the inferred information captured by SFI), we find that in practice it is simpler, both computationally and analytically, to improve the precision of SFI by expanding the basis then by performing such shape parameter optimization. We leave this possibility open for future work.

Motivated by these considerations, in practice, our choice of basis for Fig. 7 of the main text is

$$
\begin{aligned}
& b^{(1)}=\left\{x_{\mu}, \cos \theta, \sin \theta\right\}, \\
& b^{(2)}=\left\{r_{i j, \mu} r_{i j}^{k-1} \exp \left(-r_{i j} / r_{0}\right)\right\}_{k=0, \ldots, 5},
\end{aligned}
$$

where we choose $r_{0}=2$ corresponding to half the first peak in the radial distribution function. The outcome of SFI is not significantly affected by small changes in the number of functions or their shape.

\section{One-dimensional ratchet process [Figs 8(a)-8(d)]}

Figure 8 of the main text deals with the case of Brownian dynamics with multiplicative noise, i.e., with a spacedependent diffusion tensor. Figures 8 (a) -8 (d) treat a minimal example of it: a 1D ratchet process, where an out-ofequilibrium current is driven by the combination of a periodic space-dependent diffusion coefficient and a periodic force, such that the fluctuation-dissipation relation is not satisfied for a unique temperature. This model falls within the class described by Buttiker [48] and Landauer [49]. Specifically, we consider a process on the segment $[0,1]$, with periodic boundary conditions. The dynamics is described by Eq. (H2) with

$F(x)=F_{0} \cos (2 \pi x)$ and $D(x)=D_{0}+a \cos (2 \pi x)$,

where we choose $F_{0}=-2, D_{0}=1, a=0.5$, and the discretization step is $\Delta t=0.005$. The trajectory presented in Fig. 8(a) has 10000 steps.

In Figs. 8(b) and 8(c), we perform SFI on the trajectory in Fig. 8(a) using an adapted basis with $b=$ $\{1, \cos (2 \pi x), \sin (2 \pi x)\}$ for both the diffusion and the force. In Fig. 8(d), we present the convergence of the inferred fields as a function of the trajectory duration for $n=32$ repeats.

\section{Minimal 2D model with diffusion gradient [Figs. 8(e)-8(h)]}

We next consider a minimal 2D equilibrium model with inhomogeneous diffusion: an Ornstein-Uhlenbeck process with a constant gradient of isotropic diffusion coefficient. Specifically, we choose the following form for the spacedependent diffusion tensor:

$D_{\mu \nu}(\mathbf{x})=\left(1+a_{\rho} x_{\rho}\right) \delta_{\mu \nu} \quad$ with $\quad \mathbf{a}=\left(\begin{array}{c}0.25 \\ 0\end{array}\right)$,

and the following force field

$$
F_{\mu}(\mathbf{x})=-D_{\mu \nu}(\mathbf{x}) x_{\nu}
$$

corresponding to a potential well with energy $E(\mathbf{x})=\mathbf{x}^{2} / 2$ and a space-dependent mobility matrix equal to the diffusion tensor $D_{\mu \nu}(\mathbf{x})$ (i.e., the system obeys the Einstein relation with $\left.k_{B} T=1\right)$. This choice ensures that the probability distribution function of the process is unaffected by the inhomogeneity of $\mathbf{D}(\mathbf{x})$. We simulate this process using the discretized version of Eq. (8) of the main text, with $\Delta t=d t=0.02$. The trajectory in Fig. 8(e) and analyzed in Figs. 8(f) and 8(g) has length $n_{\text {steps }}=4096$. The blue symbols in Fig. 8(h) show the convergence of the diffusion estimator with increasing trajectory length $N_{\text {steps }}=$ $2^{4}, \ldots, 2^{15}$. The green and orange symbols correspond to the same data, with added measurement noise with amplitude 0.075 .

\section{Reconstruction of the drift and diffusion field for a complex 2D process (Fig. 9)}

In our last figure, we present a comparison of SFI with two preexisting methods, grid binning and InferenceMAP. To this end, we simulate a model designed to mimic the diffusion of single molecules in a complex cellular environment. To allow for quantitative comparison with the other methods, we consider here the inference of the drift field rather than the physical force, and an isotropic spacedependent diffusion tensor. The diffusion coefficient is constructed as the ratio of two second-order polynomials in the coordinates with randomly generated coefficients. The drift field is chosen as the sum of an overall harmonic trap with constant torque, three attractive Gaussian traps in a triangle, and a repulsive one at the center. Typical scales are $\Phi \sim 1, D \sim 1$, the spatial extent of the process is approximately 4 , and we choose a time step $\Delta t=0.01$. We consider two types of input signals: exact data, and noisy data where each coordinate is blurred by a Gaussian white noise of amplitude 0.1 [represented as a red dot in Fig. 9(b)].

In single-molecule contexts, the total duration of a trajectory is typically limited by photobleaching: The exploration of a cellular environment is only possible by 

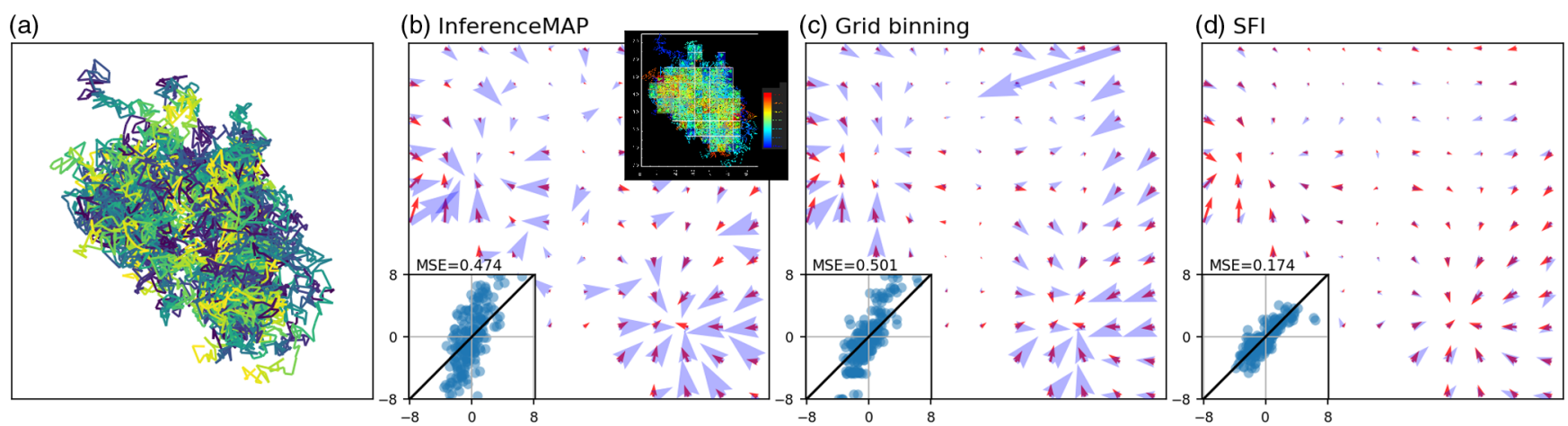

FIG. 10. Comparison of the performance of the three methods on a set of $N=128$ noisy trajectories. (a) The trajectories in the raw dataset exploited by each method. (b)-(d) The inferred drift field (thick light blue arrows) and the exact one used to generate the data (thin red arrows). The lower left insets show scatter plots of the inferred versus exact drift components, with an indication of the normalized mean-squared error (MSE). Top right inset of (b): Screen capture of the INFERENCEMAP software used for this drift field. While methods (b) and (c) capture the qualitative shape of the drift field, they appear biased, and consistently overestimate the drift for noisy data. In contrast, our method shows quantitative agreement with the exact drift field.

accumulating many such tracks. To reproduce this fact, we use $N=4, \ldots, 10^{4}$ independently generated finite-duration trajectories with 100 time steps [four of which are depicted in Fig. 9(b)], each starting at steady state. Each individual track contains, on average, an information gain of $\langle I\rangle=2.8$ bits about the drift field. We study the convergence of each method to the true drift and diffusion fields as $N \rightarrow \infty$. The performance of drift and diffusion inference are assessed as the mean-squared error between exact and inferred fields along the trajectory, normalized by the mean-squared inferred value. We now detail the parameters employed for each of the three methods.

\section{a. Stochastic force inference}

We employ a Fourier basis over a window spanning $1.1 \times$ the total process extent for both diffusion and drift inference. The order $n=1, \ldots, 9$ of the basis is adapted to the number $N$ of trajectories as $n=\lfloor\log (N)\rfloor$. We employ noise-free estimators for the exact signal and noisecorrected estimators [Eqs. (13) and (14)] for noisy data.

\section{b. Maximum-likelihood grid binning}

The principle of this method is simple: decomposing the phase space as a regular grid and inferring a constant drift vector and diffusion coefficient in each bin using maximum-likelihood estimators. The estimators are

$$
\begin{aligned}
& \hat{\Phi}(\mathbf{x})=\frac{1}{N(\mathbf{x})} \sum_{i, \mathbf{x}\left(t_{i}\right) \in \mathbf{x}} \frac{\mathbf{x}\left(t_{i+1}\right)-\mathbf{x}\left(t_{i}\right)}{\Delta t}, \\
& \hat{D}(\mathbf{x})=\frac{1}{N(\mathbf{x})} \sum_{i, \mathbf{x}\left(t_{i}\right) \in \mathbf{x}} \frac{\left[\mathbf{x}\left(t_{i+1}\right)-\mathbf{x}\left(t_{i}\right)\right]^{2}}{2 \Delta t},
\end{aligned}
$$

where the sum runs over all $N(\mathbf{x})$ data points that are inside the bin $\mathbf{x}$. We use an adaptive grid size with $n=\sqrt{N_{\text {steps }}}$ bins (width and height $\sqrt{n}$ ), where $N_{\text {steps }}$ is the total number of time points in all trajectories in the data. This ensures that both the spatial resolution and the accuracy of inference in each bin increase with the amount of data.

This method, or slight variants of it, is used in a large number of contexts $[23,33]$ and also often adapted to infer phase-space velocities $[11,14,17]$. With ideal data, we find that it performs reasonably well and converges to exact values, although not as fast as SFI. With noisy data, it becomes biased and does not converge.

\section{c. InferenceMAP}

The last method we compare to is InferenceMAP, a Bayesian method relying on space discretization introduced by Beheiry et al. [25]. This method is commonly used for the analysis of trajectories of single molecules inside cells $[6,7,24]$. We use the public implementation of this software. Upon trying many different parameters, we find that the best results are obtained with a square mesh, with maximum mesh size (the software adapts it to the amount of data), and the ( $D$, drift) inference option. We manually provide the amplitude of the measurement noise $(0$ or 0.1$)$. Typical outcome of the method is presented on Fig. 10. The performance on the inference of $D$ is slightly lower to that of SFI; it significantly outperforms grid binning in the presence of measurement noise. However, we find that the performance on drift inference does not exceed that of grid binning, and our method significantly outperforms InferenceMAP. This is demonstrated quantitatively in Fig. 9 and on an example dataset in Fig. 10.

[1] R. Brown, A Brief Account of Microscopical Observations Made in the Months of June, July and August 1827, on the Particles Contained in the Pollen of Plants; and on the 
General Existence of Active Molecules in Organic and Inorganic Bodies, Philos. Mag. 4, 161 (1828).

[2] J. Perrin, Mouvement Brownien et Réalité Moléculaire, Ann. Chim. Phys. 18, 5 (1909).

[3] L. Li, E. C. Cox, and H. Flyvbjerg, "Dicty Dynamics": Dictyostelium Motility as Persistent Random Motion, Phys. Biol. 8, 046006 (2011).

[4] B. Øksendal, Stochastic Differential Equations: An Introduction with Applications, 6th ed. (Springer-Verlag, Berlin, 2003).

[5] K. Hasselmann, Stochastic Climate Models Part I. Theory, Tellus 28, 473 (1976).

[6] S. C. Knight, L. Xie, W. Deng, B. Guglielmi, L. B. Witkowsky, L. Bosanac, E. T. Zhang, M. E. Beheiry, J.-B. Masson, M. Dahan, Z. Liu, J. A. Doudna, and R. Tjian, Dynamics of CRISPR-Cas9 Genome Interrogation in Living Cells, Science 350, 823 (2015).

[7] T. Sungkaworn, M.-L. Jobin, K. Burnecki, A. Weron, M. J. Lohse, and D. Calebiro, Single-Molecule Imaging Reveals Receptor-G Protein Interactions at Cell Surface Hot Spots, Nature (London) 550, 543 (2017).

[8] J. W. Merrill, S. K. Sainis, and E. R. Dufresne, Many-Body Electrostatic Forces between Colloidal Particles at Vanishing Ionic Strength, Phys. Rev. Lett. 103, 138301 (2009).

[9] M. Gavrilov, Y. Jun, and J. Bechhoefer, Real-Time Calibration of a Feedback Trap, Rev. Sci. Instrum. 85, 095102 (2014).

[10] D. B. Brückner, A. Fink, C. Schreiber, P. J. F. Röttgermann, J. O. Rädler, and C.P. Broedersz, Stochastic Nonlinear Dynamics of Confined Cell Migration in Two-State Systems, Nat. Phys. 15, 595 (2019).

[11] F. S. Gnesotto, F. Mura, J. Gladrow, and C. P. Broedersz, Broken Detailed Balance and Non-Equilibrium Dynamics in Living Systems: A Review, Rep. Prog. Phys. 81, 066601 (2018).

[12] Y. Roichman, B. Sun, A. Stolarski, and D. G. Grier, Influence of Nonconservative Optical Forces on the Dynamics of Optically Trapped Colloidal Spheres: The Fountain of Probability, Phys. Rev. Lett. 101, 128301 (2008).

[13] B. Lander, J. Mehl, V. Blickle, C. Bechinger, and U. Seifert, Noninvasive Measurement of Dissipation in Colloidal Systems, Phys. Rev. E 86, 030401(R) (2012).

[14] C. Battle, C. P. Broedersz, N. Fakhri, V. F. Geyer, J. Howard, C. F. Schmidt, and F.C. MacKintosh, Broken Detailed Balance at Mesoscopic Scales in Active Biological Systems, Science 352, 604 (2016).

[15] J. Gladrow, N. Fakhri, F. C. MacKintosh, C. F. Schmidt, and C. P. Broedersz, Broken Detailed Balance of Filament Dynamics in Active Networks, Phys. Rev. Lett. 116, 248301 (2016).

[16] J. Li, J. M. Horowitz, T. R. Gingrich, and N. Fakhri, Quantifying Dissipation Using Fluctuating Currents, Nat. Commun. 10, 1 (2019).

[17] D. S. Seara, V. Yadav, I. Linsmeier, A. P. Tabatabai, P. W. Oakes, S. M. A. Tabei, S. Banerjee, and M. P. Murrell, Entropy Production Rate is Maximized in Non-Contractile Actomyosin, Nat. Commun. 9, 4948 (2018).

[18] J. P. Gonzalez, J. C. Neu, and S. W. Teitsworth, Experimental Metrics for Detection of Detailed Balance Violation, Phys. Rev. E 99, 022143 (2019).
[19] U. Seifert, Stochastic Thermodynamics, Fluctuation Theorems and Molecular Machines, Rep. Prog. Phys. 75, 126001 (2012).

[20] S. Ciliberto, Experiments in Stochastic Thermodynamics: Short History and Perspectives, Phys. Rev. X 7, 021051 (2017).

[21] R. Sarfati, J. Bławzdziewicz, and E. R. Dufresne, Maximum Likelihood Estimations of Force and Mobility from Single Short Brownian Trajectories, Soft Matter 13, 2174 (2017).

[22] L. P. García, J. D. Pérez, G. Volpe, A. V. Arzola, and G. Volpe, High-Performance Reconstruction of Microscopic Force Fields from Brownian Trajectories, Nat. Commun. 9, 5166 (2018).

[23] N. Hoze, D. Nair, E. Hosy, C. Sieben, S. Manley, A. Herrmann, J.-B. Sibarita, D. Choquet, and D. Holcman, Heterogeneity of AMPA Receptor Trafficking and Molecular Interactions Revealed by Superresolution Analysis of Live Cell Imaging, Proc. Natl. Acad. Sci. U.S.A. 109, 17052 (2012).

[24] S. Türkcan, A. Alexandrou, and J.-B. Masson, A Bayesian Inference Scheme to Extract Diffusivity and Potential Fields from Confined Single-Molecule Trajectories, Biophys. J. 102, 2288 (2012).

[25] M. E. Beheiry, M. Dahan, and J.-B. Masson, InferencemaP: Mapping of Single-Molecule Dynamics with Bayesian Inference, Nat. Methods 12, 594 (2015).

[26] G. A. Gottwald, D. T. Crommelin, and C. L. E. Franzke, in Nonlinear and Stochastic Climate Dynamics, edited by C. L. E. Franzke (Cambridge University Press, Cambridge, England, 2017), pp. 209-240.

[27] F. Böttcher, J. Peinke, D. Kleinhans, R. Friedrich, P. G. Lind, and M. Haase, Reconstruction of Complex Dynamical Systems Affected by Strong Measurement Noise, Phys. Rev. Lett. 97, 090603 (2006).

[28] E. Gobet, M. Hoffmann, and M. Reiß, Nonparametric Estimation of Scalar Diffusions Based on Low Frequency Data, Ann. Stat. 32, 2223 (2004).

[29] F. Comte, V. Genon-Catalot, and Y. Rozenholc, Penalized Nonparametric Mean Square Estimation of the Coefficients of Diffusion Processes, Bernoulli 13, 514 (2007).

[30] M. Hoffmann, Adaptive Estimation in Diffusion Processes, Stoch. Proc. Appl. 79, 135 (1999).

[31] Y. A. Kutoyants, Statistical Inference for Ergodic Diffusion Processes, Springer Series in Statistics (Springer-Verlag, London, 2004).

[32] O. Papaspiliopoulos, Y. Pokern, G. O. Roberts, and A. M. Stuart, Nonparametric Estimation of Diffusions: A Differential Equations Approach, Biometrika 99, 511 (2012).

[33] R. Friedrich, J. Peinke, M. Sahimi, and M. R. R. Tabar, Approaching Complexity by Stochastic Methods: From Biological Systems to Turbulence, Phys. Rep. 506, 87 (2011).

[34] T. M. Cover and J. A. Thomas, Elements of Information Theory, Wiley Series in Telecommunications and Signal Processing (Wiley-Interscience, New York, 2006).

[35] P. Batz, A. Ruttor, and M. Opper, Variational Estimation of the Drift for Stochastic Differential Equations from the Empirical Density, J. Stat. Mech. (2016) 083404.

[36] M. Baiesi and G. Falasco, Inflow Rate, a Time-Symmetric Observable Obeying Fluctuation Relations, Phys. Rev. E 92, 042162 (2015). 
[37] C. Maes, K. Netočný, and B. Wynants, Steady State Statistics of Driven Diffusions, Physica (Amsterdam) 387A, 2675 (2008).

[38] R. Chetrite and K. Gawędzki, Fluctuation Relations for Diffusion Processes, Commun. Math. Phys. 282, 469 (2008).

[39] H. Risken and T. Frank, The Fokker-Planck Equation: Methods of Solution and Applications, 2nd ed., Springer Series in Synergetics (Springer-Verlag, Berlin, 1996).

[40] R. Chetrite and K. Gawędzki, Eulerian and Lagrangian Pictures of Non-Equilibrium Diffusions, J. Stat. Phys. 137, 890 (2009).

[41] A. Ghanta, J. C. Neu, and S. Teitsworth, Fluctuation Loops in Noise-Driven Linear Dynamical Systems, Phys. Rev. E 95, 032128 (2017).

[42] M. S. Shkarayev and R. K. P. Zia, Exact Results for a Simple Epidemic Model on a Directed Network: Explorations of a System in a Nonequilibrium Steady State, Phys. Rev. E 90, 032107 (2014).

[43] R. K. P. Zia, J. B. Weiss, D. Mandal, and B. Fox-Kemper, Manifest and Subtle Cyclic Behavior in Nonequilibrium Steady States, J. Phys. Conf. Ser. 750, 012003 (2016).

[44] F. Mura, G. Gradziuk, and C. P. Broedersz, Nonequilibrium Scaling Behavior in Driven Soft Biological Assemblies, Phys. Rev. Lett. 121, 038002 (2018).

[45] É. Roldán, J. Barral, P. Martin, J. M. R. Parrondo, and F. Jülicher, Arrow of Time in Active Fluctuations, arXiv: 1803.04743 .

[46] A. Allawala and J. B. Marston, Statistics of the Stochastically Forced Lorenz Attractor by the Fokker-Planck Equation and Cumulant Expansions, Phys. Rev. E 94, 052218 (2016).

[47] A. W. C. Lau and T. C. Lubensky, State-Dependent Diffusion: Thermodynamic Consistency and Its Path Integral Formulation, Phys. Rev. E 76, 011123 (2007).

[48] M. Büttiker, Transport as a Consequence of StateDependent Diffusion, Z. Phys. B 68, 161 (1987).

[49] R. Landauer, Motion Out of Noisy States, J. Stat. Phys. 53, 233 (1988).

[50] C. L. Vestergaard, P. C. Blainey, and H. Flyvbjerg, Optimal Estimation of Diffusion Coefficients from Single-Particle Trajectories, Phys. Rev. E 89, 022726 (2014).

[51] C. Penland and T. Magorian, Prediction of Niño 3 Sea Surface Temperatures Using Linear Inverse Modeling, J. Clim. 6, 1067 (1993).

[52] J.P. N. Bishwal, Parameter Estimation in Stochastic Differential Equations, Lecture Notes in Mathematics (Springer-Verlag, Berlin, 2008).
[53] D. Crommelin and E. Vanden-Eijnden, Diffusion Estimation from Multiscale Data by Operator Eigenpairs, Multiscale Model. Simul. 9, 1588 (2011).

[54] A. Ruttor, P. Batz, and M. Opper, in Advances in Neural Information Processing Systems 26, edited by C. J. C. Burges, L. Bottou, M. Welling, Z. Ghahramani, and K. Q. Weinberger (Curran Associates, Inc., 2013), pp. 20402048.

[55] C. Yildiz, M. Heinonen, J. Intosalmi, H. Mannerstrom, and H. Lahdesmaki, in Proceedings of the 2018 IEEE 28th International Workshop on Machine Learning for Signal Processing (MLSP) (IEEE, New York, 2018), pp. 1-6.

[56] A. C. Barato and U. Seifert, Thermodynamic Uncertainty Relation for Biomolecular Processes, Phys. Rev. Lett. 114, 158101 (2015).

[57] J. O. Daldrop, J. Kappler, F. N. Brünig, and R. R. Netz, Butane Dihedral Angle Dynamics in Water is Dominated by Internal Friction, Proc. Natl. Acad. Sci. U.S.A. 115, 5169 (2018).

[58] J. Palacci, S. Sacanna, A. P. Steinberg, D. J. Pine, and P. M. Chaikin, Living Crystals of Light-Activated Colloidal Surfers, Science 339, 936 (2013).

[59] A. Bricard, J.-B. Caussin, N. Desreumaux, O. Dauchot, and D. Bartolo, Emergence of Macroscopic Directed Motion in Populations of Motile Colloids, Nature (London) 503, 95 (2013).

[60] A. Celani and M. Vergassola, Bacterial Strategies for Chemotaxis Response, Proc. Natl. Acad. Sci. U.S.A. 107, 1391 (2010).

[61] G. J. Stephens, B. Johnson-Kerner, W. Bialek, and W. S. Ryu, Dimensionality and Dynamics in the Behavior of C. elegans, PLoS Comput. Biol. 4, e1000028 (2008).

[62] M. C. Wheeler and H. H. Hendon, An All-Season Real-Time Multivariate MJO Index: Development of an Index for Monitoring and Prediction, Mon. Weather Rev. 132, 1917 (2004).

[63] S. L. Brunton, J. L. Proctor, and J. N. Kutz, Discovering Governing Equations from Data by Sparse Identification of Nonlinear Dynamical Systems, Proc. Natl. Acad. Sci. U.S.A. 113, 3932 (2016).

[64] https://github.com/ronceray/StochasticForceInference

[65] C. Gardiner, Stochastic Methods: A Handbook for the Natural and Social Sciences, 4th ed., Springer Series in Synergetics (Springer-Verlag, Berlin, 2009).

[66] H. Touchette, Introduction to Dynamical Large Deviations of Markov Processes, Physica (Amsterdam) 504A, 5 (2018). 